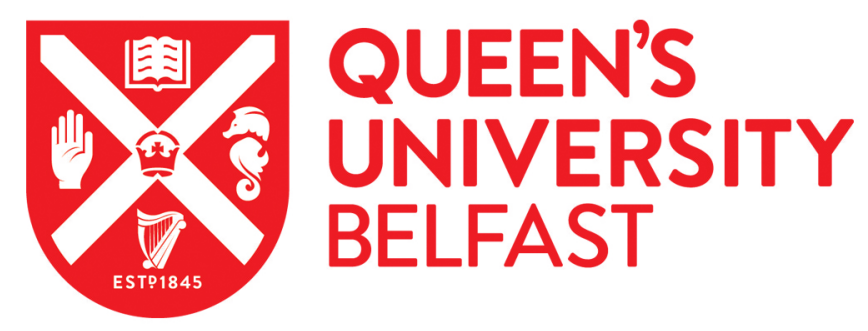

\title{
Conservative fluid management or deresuscitation for patients with sepsis or acute respiratory distress syndrome following the resuscitation phase of critical illness: a systematic review and meta- analysis
}

Silversides, J. A., Major, E., Ferguson, A. J., Mann, E. E., McAuley, D. F., Marshall, J. C., Blackwood, B., \& Fan, E. (2016). Conservative fluid management or deresuscitation for patients with sepsis or acute respiratory distress syndrome following the resuscitation phase of critical illness: a systematic review and meta-analysis. Intensive Care Medicine. https://doi.org/10.1007/s00134-016-4573-3

Published in:

Intensive Care Medicine

Document Version:

Peer reviewed version

Queen's University Belfast - Research Portal:

Link to publication record in Queen's University Belfast Research Portal

Publisher rights

(C) Springer-Verlag Berlin Heidelberg and ESICM 2016

The final publication is available at Springer via http://link.springer.com/article/10.1007/s00134-016-4573-3

\section{General rights}

Copyright for the publications made accessible via the Queen's University Belfast Research Portal is retained by the author(s) and / or other copyright owners and it is a condition of accessing these publications that users recognise and abide by the legal requirements associated with these rights.

Take down policy

The Research Portal is Queen's institutional repository that provides access to Queen's research output. Every effort has been made to ensure that content in the Research Portal does not infringe any person's rights, or applicable UK laws. If you discover content in the Research Portal that you believe breaches copyright or violates any law, please contact openaccess@qub.ac.uk. 
Conservative fluid management or deresuscitation for patients with sepsis or acute respiratory distress syndrome following the resuscitation phase of critical illness: a systematic review and meta-analysis

Jonathan A Silversides ${ }^{1,2^{*}}$, Emmet Major ${ }^{2}$, Andrew J Ferguson ${ }^{3}$, Emma E Mann², Daniel F McAuley ${ }^{1,4}$, John C Marshall ${ }^{5,6}$, Bronagh Blackwood ${ }^{1 \#}$, Eddy Fan ${ }^{5 \#}$

1. Centre for Experimental Medicine

Wellcome-Wolfson Institute

Queen's University of Belfast

97 Lisburn Road

Belfast BT9 7BL

United Kingdom

2. Department of Critical Care Services

Belfast Health and Social Care Trust

Belfast City Hospital

Lisburn Road

Belfast BT9 7AB

United Kingdom

3. Department of Intensive Care

Southern Health and Social Care Trust

Craigavon Area Hospital

68 Lurgan Road

Portadown BT63 5QQ

United Kingdom

4. Regional Intensive Care Unit

Department of Critical Care Services

Belfast Health and Social Care Trust

Royal Victoria Hospital

Grosvenor Road

Belfast BT12 6BA

United Kingdom

5. Interdepartmental Division of Critical Care

University of Toronto

585 University Avenue

PMB 11-123

Toronto, Ontario, M5G 2N2

Canada

6. Department of Critical Care Medicine

St Michael's Hospital

30 Bond Street,

Bond 4-014

Toronto, Ontario, M5B 1W8

Canada

*Corresponding author

Email: jsilversides01@qub.ac.uk

Telephone: +44 2895041101

Facsimile: +44 2890263892

\# These authors contributed equally to the work

On behalf of all authors, the corresponding author states that there are no conflicts of interest. 


\begin{abstract}
Background: It is unknown whether a conservative approach to fluid administration or deresuscitation (active removal of fluid using diuretics or renal replacement therapy (RRT)) is beneficial following haemodynamic stabilisation of critically ill patients.
\end{abstract}

Purpose: To evaluate the efficacy and safety of conservative or deresuscitative fluid strategies in adults and children with acute respiratory distress syndrome (ARDS), sepsis, or systemic inflammatory response syndrome (SIRS) in the post-resuscitation phase of critical illness.

Methods: We searched Medline, EMBASE, and the Cochrane central register of controlled trials from 1980 to June 2016, and manually reviewed relevant conference proceedings from 2009 to the present. Two reviewers independently assessed search results for inclusion and undertook data extraction and quality appraisal. We included randomised trials comparing fluid regimens with differing fluid balances between groups, and observational studies investigating the relationship between fluid balance and clinical outcomes.

Results: 49 studies met inclusion criteria. Marked clinical heterogeneity was evident. In a meta-analysis of 11 randomised trials (2051 patients) using a random effects model, we found no significant difference in mortality with conservative or deresuscitative strategies compared to a liberal strategy or usual care (pooled risk ratio $[\mathrm{RR}] 0.92,95 \%$ confidence interval $[\mathrm{Cl}] 0.82-1.02, \mathrm{I}^{2}=0 \%$ ). A conservative or deresuscitative strategy resulted in increased ventilator-free days (mean difference 1.82 days, 95\% $\mathrm{Cl} 0.53$ to 3.10 days, $I^{2}=9 \%$ ) and reduced length of ICU stay (mean difference -1.88 days, $95 \% \mathrm{Cl}-0.12$ to -3.64 days, $I^{2}=75 \%$ ) compared to a liberal strategy or standard care.

Conclusions: In adults and children with ARDS, sepsis or SIRS, a conservative or deresuscitative fluid strategy results in increased number of ventilator-free days and decreased length of ICU stay compared with a liberal strategy or standard care. The effect on mortality remains uncertain. Large randomised trials are needed to determine optimal fluid strategies in critical illness.

Keywords: Fluid therapy; Diuretics; Water-electrolyte balance; Critical Illness; Sepsis; Respiratory Distress Syndrome, Adult; Systemic Inflammatory Response Syndrome. 


\section{Background}

Optimising fluid status is a fundamental concern of critical care practice. Ample data suggest that the optimisation of intravascular volume status can increase cardiac output and global oxygen delivery, and large volumes of intravenous fluids are often administered for this purpose. In addition, critically ill patients frequently receive large volumes of fluid as drug diluents, as artificial nutrition, and as maintenance fluid.

In the face of increased capillary permeability, sodium and water retention, and acute kidney injury (AKI), all of which are common in critical illness, the accumulation of large volumes of fluid in the interstitium is a frequent occurrence and may impair oxygen delivery at the cellular level. Clinically this fluid overload is apparent as peripheral and pulmonary oedema, although other organs may be affected [1]. A number of cohort studies have demonstrated an association between fluid overload and mortality [2-4], and it has been suggested that strategies aimed at prevention or treatment of fluid overload may be beneficial following haemodynamic stabilisation [5].

A previous systematic review and meta-analysis on the topic of fluid overload and the relationship between fluid balance and mortality [6] in critically ill patients reported studies with considerable heterogeneity in design, presence of comparator groups, populations, as well as the timing and nature of interventions. By narrowing our focus to specific populations, and by including but not attempting to meta-analyse observational studies, we aimed to maximise both the external and internal validity of our review.

The aim of this review is to evaluate the impact of a conservative fluid or active deresuscitation strategy compared with standard care or a liberal fluid strategy in critically ill adult or paediatric patients with sepsis, systemic inflammatory response syndrome (SIRS), or acute respiratory distress syndrome (ARDS) on mortality and other clinical outcomes. Secondary aims were to identify criteria used to judge suitability for conservative fluid management or deresuscitation; to describe the interventions used to minimise fluid intake or deresuscitate patients, and to identify contraindications to deresuscitation or conservative fluid management in published studies.

\section{Methods}

The protocol for this review was prospectively registered with PROSPERO (International prospective register of systematic reviews; CRD42013005608) and published previously [7]. We used Cochrane review methodology [8] in protocol development and review conduct, and adhered to the Preferred Reporting Items for Systematic Reviews and Meta-Analyses (PRISMA) guidelines [9] in reporting the review.

\section{Search strategy}

MEDLINE, EMBASE and the Cochrane Central register of controlled trials (CENTRAL) were searched (up to $24^{\text {th }}$ June, 2016) for potentially relevant studies without language constraints. In addition, we manually searched indexed abstracts from the American Thoracic Society, Society of Critical Care Medicine, and European Society of Intensive Care medicine annual congresses and the International Symposium on Intensive Care and Emergency Medicine from 2009 to the present. A full list of MEDLINE search terms is available as an appendix to the published protocol [7].

\section{Inclusion and exclusion criteria}

We included randomised and quasi-randomised clinical trials of adult or paediatric patients with ARDS, SIRS or sepsis in which two or more fluid strategies were compared and in which fluid balance differed between groups; and observational studies in which the relationship between fluid balance and clinical outcomes in ARDS, SIRS or sepsis was the major focus of the study.

We excluded studies that focused only on the resuscitation phase of critical illness, and studies in which fluids were only one element of a complex haemodynamic strategy. We also excluded case series, case reports, observational studies with fewer than 50 participants, studies published prior to 1980, studies involving predominantly neonates, post-cardiac surgery patients, or patients with heart failure, and studies subject to post-publication retraction or investigation. 


\section{Selection of studies and data extraction}

Titles and abstracts of all reports identified in the literature searches were screened by two of three authors (JS, EEM and AF) for further review with discrepancies resolved by consensus. Full text review of eligibility was conducted by two authors independently (JS and EM) and relevant data extracted in duplicate from included studies to a standard piloted form [7]. Discrepancies were resolved by discussion and adjudication by a third author (EF). Where relevant, attempts were made to contact authors of randomised studies for missing data. The reference lists of included randomised trials were reviewed for additional trials meeting eligibility criteria.

\section{Outcome measures}

The primary outcome was all-cause mortality at the latest time point available up to 90 days. Key secondary outcomes included ventilator-free days (VFDs), length of intensive care unit (ICU) stay, incidence of $\mathrm{AKI}$, renal replacement therapy (RRT) use, and cognitive impairment.

\section{Risk of bias assessment}

Two authors (JS and EM) independently assessed risk of bias and quality. Randomised controlled trials were assessed as being at low, uncertain or high risk of bias for each of 6 domains using the Cochrane risk of bias tool [8]. Cohort and case-control studies were assessed for quality using the Newcastle Ottawa scale [10] (Appendix 2).

\section{Analysis}

RevMan software [8] was used to carry out meta-analysis using a random effects model for outcomes for which two or more randomised studies were available. Results for outcomes for which meta-analysis was deemed inappropriate because of an insufficient number of studies or clinical or statistical heterogeneity were reported in narrative form, and observational studies were reported in tabular form (Appendix 1). Where necessary to standardise reporting of central tendency between studies, we converted standard error to standard deviation, and estimated mean and standard deviation from reported median and interquartile ranges using a standard approach [11]. For key outcomes, we assessed the quality of evidence using the Grades of Recommendation, Assessment, Development and Evaluation (GRADE) approach [12].

We undertook a pre-planned sensitivity analysis excluding studies at high risk of bias, and subgroup analyses for ARDS, sepsis or SIRS, and adults. We also undertook a post-hoc analysis in which we excluded studies lacking a clinically-significant difference in fluid balance between groups, which we defined as a minimum difference in mean or median fluid balance of $750 \mathrm{~mL}$ per day for adults or 10 $\mathrm{mL} / \mathrm{kg} / \mathrm{day}$ for children. We also carried out a meta-regression analysis with difference in mean daily fluid balance as the independent, and risk ratio (RR) for mortality as the dependent variable.

\section{Results}

The search was conducted up to 24 June 2016 and during the editorial process we obtained one further study in press from the editor. Forty-nine studies met criteria for inclusion. Of these, 11 randomised controlled trials, recruiting a total of 2051 patients, provided data for meta-analysis. The remaining 38 studies were observational in design and are summarised in Appendix 1. The Newcastle-Ottawa score for observational studies is reported in Appendix 2. Secondary publications from included studies are reported along with the original study [13-15]. A summary of evidence is found in Table 2.

\section{Description of included randomised trials}

Considerable clinical heterogeneity was present. Five studies [16-20] took place in the United States, three in China [21-23], one in France [24] one in India [25] and one in Denmark and Finland [26]. Sample sizes ranged from 29 [21] to 1000 [16]. One was conducted in children [25] and the remainder in adults. Five studies included only patients with ARDS [16-18, 21, 22], four included only patients with septic shock [19, 24-26]; one included patients with ARDS, septic shock, or both [23] and one included a mixed critically ill 
population, the majority of whom had sepsis, ARDS, or both [20]. Further characteristics of included randomised trials are presented in Table 1.

\section{Methodological quality and risk of bias}

The overall quality of included randomised trials was moderate (Figure 2). The use of random sequence generation and allocation concealment $[19-22,25]$ and the risk of reporting bias [18, 20-22, 25] were unclear in a number of studies. While blinding was used in only 2 studies [17, 18], likely due to difficulties in concealment of the different fluid regimens and/or haemodynamic monitoring technologies employed, strict protocolisation of fluid and diuretic use was felt to ameliorate the effects of this potential bias in all but two studies $[19,21]$.

\section{Mortality (primary outcome)}

Eleven studies (2051 patients) reported mortality as an outcome with variable duration of follow-up, including 90-day [26], 60-day [16, 21, 22], in-hospital [19, 20] and 28 or 30-day mortality [17, 18, 23-25]. We found no significant difference in mortality between patients receiving a conservative or deresuscitative fluid strategy compared with those receiving a liberal strategy or standard care (pooled RR 0.92; 95\% confidence interval $[\mathrm{Cl}]$ 0.82-1.02, $\mathrm{I}^{2}=0 \%$ ) (Figure 3).

One trial [16] accounted for the majority of patients in the ARDS subgroup, and the results for this subgroup ( 5 studies, $\mathrm{n}=1206$, pooled RR $0.91 ; 95 \% \mathrm{Cl} 0.77-1.07$ ) were similar to those in the overall analysis. In the sepsis/SIRS subgroup, three trials were conducted in adults $[19,24,26]$ and one in children [25]. Results from this subgroup analysis were also similar to those in the overall analysis (394 patients, pooled RR 0.86; 95\% Cl 0.62-1.17) (Figure 3).

\section{Secondary outcomes}

Ventilator-free days

Data on the number of VFDs within a 28 or 30-day period were available for seven studies, including 1784 participants (Figure 4). We found increased VFDs with a conservative or deresuscitative fluid strategy in comparison with a liberal strategy or standard care (mean difference 1.82 days [95\% Cl interval 0.53 to 3.10 days], $I^{2}=9 \%$ ). In addition, studies by Hu et al [21] and Wang et al [22] reported shorter duration of mechanical ventilation in a more conservative fluid strategy group compared with the liberal fluid strategy group $(10.13+/-3.02$ days vs. $12.64+/-2.89, \mathrm{P}<0.05$ and $9.62+/-2.55$ days vs $12.51+/-2.92$ days, $\mathrm{P}<0.05$ respectively).

\section{Length of ICU stay}

Nine studies reported the duration of ICU admission of which seven were suitable for meta-analysis (Figure 5). We found a shorter length of ICU stay in patients receiving a conservative or deresuscitative fluid strategy compared with those receiving a liberal strategy or standard care (mean difference 1.88 days fewer ( $95 \% \mathrm{Cl}-0.12$ to -3.64 days). Considerable heterogeneity was present $\left(I^{2}=75 \%\right)$. Two studies in ARDS patients reported a composite outcome of ICU-free days: Martin et al [18] reported a numerically greater number of ICU-free days in the fluid conservative group (median 1.5 days greater, $95 \% \mathrm{Cl}-3.4$ to +6.4 days), while in the Fluids and Catheter Treatment Trial (FACTT) [16], a conservative strategy resulted in a significantly greater number of ICU-free days compared to a liberal strategy $(13.4+/-8.97$ versus 11.2 $+/-8.92, \mathrm{P}<0.001)$.

Length of Hospital stay

One study [18] reported no significant reduction in the length of hospital stay for survivors of ARDS with a deresuscitative strategy (median 4.5 fewer days in hospital, $95 \% \mathrm{Cl}-5.8$ to 14.8 days).

Organ dysfunction scores

Martin et al [17] reported a fall in mean Sequential Organ Failure Assessment (SOFA) score of 0.6 with a deresuscitation strategy compared with an increase of 1.1 in the control group over the 5 day study period $(P=0.01)$. Zhang et al [23] reported higher maximum SOFA scores in the more conservatively managed group, although this difference was also present at baseline; and Richard et al [24] reported similar duration of SOFA score $\geq 6$. 
Long-term mortality

No studies reported long-term ( $>90$ day) mortality as an outcome.

Incidence of ARDS

No studies reported incidence of ARDS as an outcome.

Incidence of Acute Kidney Injury

Martin et al [18] reported no difference in change in serum creatinine between patients in a deresuscitation group compared with placebo, while in the FACTT study [16] the incidence of AKI was similar between conservative and liberal fluid management groups $(21.5+/-11.21$ renal failure free days versus $21.2+/$ 11.15, $\mathrm{P}=0.59$ ). Hjortrup et al [26] reported a lower incidence of worsening of AKI in a conservative fluid group than with standard care ( $37 \%$ versus $54 \%, P=0.03$ ). In separate post-hoc analyses of the FACTT study, Liu and colleagues showed that after correcting serum creatinine levels for fluid balance, AKI incidence was lower with a conservative than with a liberal fluid strategy [14]; and Grams et al reported that in patients with AKI, cumulative diuretic dose was independently associated with lower mortality [15].

Renal replacement therapy use

In three studies $[16,19,26]$ (1233 patients), the rate of RRT use was similar between patients receiving a conservative fluid or deresuscitative strategy compared with a liberal fluid strategy or standard care (RR $0.88 ; 95 \% \mathrm{Cl} 0.64-1.22, \mathrm{I}^{2}=27 \%$ ) (Appendix 3.5). Zhang et al [23] reported fewer days free of continuous RRT in the conservative fluid strategy group (median 15.5 days [IQR 3-28] versus 21 [4-28], $\mathrm{P}<0.05$ ).

Cognitive function

In a cohort of seventy-five survivors from FACTT [16] who underwent follow up assessment of cognitive function, Mikkelsen et al [13] identified enrolment in the conservative fluid management arm as an independent risk factor for cognitive impairment at twelve months post hospital discharge. In contrast, Wang and colleagues [22] assessed post-ICU cognitive function as one component of the QLQ-C30 quality of life score, and found better cognitive function scores in patients treated with a conservative fluid strategy than a liberal fluid strategy (85.02 +/- 15.06 vs. $74.31+/-12.88, P<0.05)$.

\section{Additional analyses}

Additional sensitivity and subgroup analyses are found in Appendix 3.

\section{Readiness for conservative fluid management or deresuscitation}

The majority of studies did not attempt to use specific physiological or time criteria to determine readiness for conservative fluid management or deresuscitation. One study [19] postponed initiation of a conservative fluid management strategy until patients were demonstrated to be volume unresponsive. Fluid minimisation occurred between one and four days post-randomisation, however clinically-significant separation of fluid balance between groups was not achieved over five days.

\section{Interventions}

There was considerable variation in fluid strategies applied and fluid balances achieved in both conservative / deresuscitative and liberal / standard care groups. In three studies [16-18], protocolised diuretic use was used in the conservative / deresuscitative arm, in four the intervention strategy involved protocolised fluid restriction or minimisation $[16,19,25,26]$; and in five the main intervention was the use of alternative haemodynamic targets for fluid management, based on extravascular lung water (EVLW) [2022], pulse pressure variation (PPV) [24], or intrathoracic blood volume index (ITBVI) [23]. In two trials hyperoncotic albumin infusions were used to potentiate diuresis in a deresuscitative group $[17,18]$. Fluid strategies in study control arms included protocolised liberal fluid administration [16], protocolised diuretic use without hyperoncotic albumin [17] and central venous pressure (CVP) or pulmonary capillary wedge pressure (PCWP)-guided fluid administration [20, 21, 23, 24].

As a result of variability in fluid strategies used, there was wide variation in fluid balances and considerable overlap between conservative and liberal groups. For example, in the study by Martin et al [17] the 'liberal' group received diuretics and achieved a weight loss of $4700 \mathrm{~mL}$ over five days, equating to an estimated mean fluid balance of $-22.4 \mathrm{~mL} / \mathrm{kg} / \mathrm{day}$; while in the study by Chen and Kollef [19], a targeted fluid 
minimisation strategy in the conservative arm yielded a median positive fluid balance of $2641 \mathrm{~mL}$ over five days, equating to a positive mean fluid balance of $7.5 \mathrm{~mL} / \mathrm{kg} /$ day.

\section{Contraindications to deresuscitative fluid management}

Two studies of deresuscitation $[17,18]$ excluded patients with AKI, those with more than a minimal requirement for vasopressors, and those with uncorrected hypernatraemia or hypokalaemia.

Deresuscitation was suspended if hypotension, hypernatraemia or hypokalaemia developed during the intervention period, and fluid boluses were given at the discretion of the clinical team. In FACTT [16], fluid administration and diuretic use was protocolised, so that haemodynamic insufficiency triggered fluid bolus administration or vasoactive medication use, and diuretics were withheld in the presence of AKI.

\section{Observational studies}

We included a total of 38 observational studies in this review; characteristics are reported in appendix 1. The majority were cohort studies in which fluid balance was compared between survivors and nonsurvivors of critical illness, with or without adjustment for severity of illness and other potential confounders. The majority of observational studies were assessed as moderate or low quality using the NewcastleOttawa scale (Appendix 2).

The main finding was a consistent positive association between more positive fluid balance and higher mortality $[3,4,27-52]$ which was present within all pre-specified subgroups: adults $[3,4,28,30-33,36-38$, 40-46, 48, 50-53], children [27, 29, 35, 49], ARDS [3, 32, 35, 39, 40, 43, 46, 48, 49] and sepsis [4, 27-31, $33-38,40-42,44,45,50-53$. This association was absent or present only in subgroups in seven studies in which mortality was reported as an outcome [54-60]. One study reported a lower mortality with greater fluid administration and more positive fluid balance over 3 days [61]. A more positive fluid balance was associated with increased $[32,55]$ or similar $[29,42]$ duration of mechanical ventilation, fewer ventilator-free days $[35,54,56,60]$ and increased $[32,52,60]$ or similar $[42,55]$ length of ICU stay. Rates of AKI or RRT use were similar $[29,33,56,59,61,62]$ or higher $[36,60]$ with a more positive fluid balance.

\section{Discussion}

Although reference is made in current guidelines to the use of intravenous fluid for resuscitation in sepsis [63], fluid management goals following the resuscitation phase of critical illness remain the subject of considerable uncertainty. Our review evaluated the efficacy and safety of a conservative or deresuscitative fluid strategy compared with standard care or a liberal fluid strategy in critically ill patients with sepsis, SIRS, or ARDS.

We found no clear evidence for the superiority of one fluid strategy over another for our primary outcome of mortality. This is in contrast to a previous meta-analysis [6], and likely reflects our exclusion of observational data from our meta-analysis. We found that a conservative or deresuscitative fluid strategy resulted in a greater number of VFDs and decreased length of ICU stay than a liberal fluid strategy or standard care, with no increase in acute kidney injury, use of RRT, or cognitive dysfunction. When we excluded those studies in which we considered inter-group differences in fluid balance to be clinically unimportant, we found a non-significant reduction in mortality with conservative or deresuscitative fluid management (Appendix 3.3). The quality of evidence was low or very low across all outcomes.

We found no difference in rates of renal replacement therapy use between fluid strategies. Along with posthoc analyses of the FACTT study showing a reduced incidence of AKI with a conservative fluid strategy [14] and a protective effect of diuretic use [15], this provide reassurance as to the safety of a conservative or deresuscitative approach to fluid management in terms of renal outcomes.

The effect of a conservative fluid strategy or deresuscitation in terms of cognitive outcomes is unclear, with a secondary analysis of a small cohort of patients from the FACTT study showing evidence of harm from a conservative approach [13]. This contrasts with the findings of Wang and colleagues in which post-ICU discharge cognitive function was improved in a conservative fluid management group [22], and those of a small randomised trial in patients undergoing major vascular surgery where a conservative fluid strategy was associated with a reduction in post-operative complications including delirium [64], a clinical outcome 
known to be associated with longer term cognitive dysfunction [65]. This merits further investigation in future trials investigating fluid strategy.

Our review has a number of strengths. It was conducted using high quality systematic review methodology. A highly sensitive search strategy was developed which was independently reviewed by a second information specialist. In order to minimise bias, no language restrictions were employed, and broad date criteria were applied. At least two reviewers were involved independently at each stage of the review process, and all studies were evaluated for quality and risk of bias.

There are a number of important limitations in this review, however. Even in the small number of studies included, considerable heterogeneity was evident with respect to study populations, interventions, and outcomes. Due to lack of standardised definitions, the timing and duration of the 'post-resuscitation' intervention period varied between studies, although the available data did not allow in-depth exploration of this issue. This highlights the need to standardise these definitions for future clinical trials. Because of insufficient data, we were unable to separate the differential impact of restrictive fluid administration and active deresuscitation. Some of the interventions employed resulted in minimal separation between groups in fluid balance. As we did not define what constituted a clinically-significant difference in fluid balance between groups a priori, we included all in our main analysis (Figure 3) but undertook a sensitivity analysis in which studies were excluded on the basis of clinically insignificant differences in fluid balance between groups (Appendix 3.3).

There was considerable inconsistency in reporting which precluded some studies for inclusion in metaanalyses, exemplified by some studies reporting duration of mechanical ventilation with others reporting a composite outcome of ventilator-free days. This is a recognised problem in studies of patients receiving mechanical ventilation [66]. Even for the uniformly reported outcome of mortality, there was variability in the duration of follow-up from 28 to 90 days, although this is unlikely to have had a major impact on summary estimates of effect [67].

We limited our review to patients with sepsis, SIRS and ARDS. The inevitable consequence is a loss of generalizability to other types of critically ill patients, although since these are common syndromes rather than specific diagnoses, and since patients admitted to ICU with a range of pathologies (e.g. traumatic brain injury [68] and polytrauma [69]) frequently develop SIRS, ARDS and sepsis, the generalizability of these findings is likely go beyond simply those patients who meet rigidly applied consensus criteria.

We identified a large number of observational studies in which fluid accumulation or overload was associated with worse outcomes, particularly mortality. The potential for residual confounding is present to some extent in all of these, in that greater cumulative fluid balances may reflect greater severity of illness and greater perceived or actual need for fluid resuscitation or clinician reluctance to either withhold fluid or to administer diuretics to more severely ill patients.

Robust multicentre trials are needed to evaluate the effectiveness of restrictive fluid administration, deresuscitation or a combined fluid strategy to improve patient outcomes. Based on our data, a sample size of over 4700 patients would be required to detect or exclude a significant mortality benefit for a conservative and/or deresuscitative fluid strategy (Appendix 3.3). However, the heterogeneity illustrated in this review highlights the need for considerable further pilot work to define the optimal intervention strategy or strategies to be subsequently tested in high-quality, adequately powered multicentre randomised trials. Pilot studies should, for example, address the questions of physiological or other criteria to define the appropriate timing for conservative fluid management, the utility of deresuscitation in addition to fluid restriction alone, the comparative benefits and harms of ultrafiltration and diuretics, and the use of adjunctive hypertonic albumin among others.

\section{Conclusions}

Despite a considerable body of observational evidence showing a positive association between fluid balance and mortality, our review found no significant difference in mortality from included randomised trials addressing the question of optimal fluid strategy for critically ill patients. We found that a conservative or deresuscitative approach resulted in increased ventilator-free days and decreased length of ICU stay compared to a liberal strategy or standard care. 
Large robust trials are needed in which clear inter-group differences in fluid balance are present to evaluate the efficacy and safety of a conservative or deresuscitative fluid strategy in terms of both short and long term outcomes. The optimum strategy to be tested in such trials remains to be defined. Meanwhile, clinicians caring for critically ill patients may consider the use of a conservative fluid management strategy in patients with sepsis, ARDS and SIRS following initial resuscitation and stabilisation.

\section{Acknowledgements}

The authors wish to acknowledge the invaluable assistance of information specialists Ms Viola Machel, Ms Melanie Anderson, and Ms Marina Englesakis (University Health Network) and Richard Fallis (Queen's University of Belfast) in the development and implementation of the search strategies; Ms Adrienne Ruddock for assistance with image preparation, and Dr Hong Guo Parke, Dr Edmund Skibowski and Ms Chuer Zhang for translation of non-English manuscripts.

This work was supported by a doctoral fellowship award to JS by the Northern Ireland Health and Social Care research and development division.

\section{Supplementary Material}

Appendix 1 - Characteristics and key findings of included observational studies

Appendix 2 - Assessment of study quality (modified Newcastle Ottawa scale) for observational studies

Appendix 3 - Additional analyses: (3.1) Pre-planned sensitivity analysis excluding studies at high or moderate risk of bias with mortality as outcome. (3.2) Pre-planned subgroup analysis including only adult studies with mortality as outcome. (3.3) Post-hoc sensitivity analysis excluding studies lacking a clinicallyimportant separation in fluid balance between groups (3.4) Univariate meta-regression analysis with RR for mortality as dependent variable and between-group difference in mean daily fluid balance as exposure. $\mathrm{R}^{2}=0.11, \mathrm{P}=0.30$. (3.5) Forest plot for renal replacement therapy use, conservative or deresuscitative fluid strategy versus standard care or liberal fluid strategy.

Appendix 4 - List of excluded studies

\section{References}

1. Prowle JR, Echeverri JE, Ligabo EV, et al (2009) Fluid balance and acute kidney injury. Nat Rev Nephrol 6:107-115.

2. Payen D, de Pont A-CJM, Sakr Y, et al (2008) A positive fluid balance is associated with a worse outcome in patients with acute renal failure. Crit Care 12:R74.

3. Rosenberg AL, Dechert RE, Park PK, et al (2008) Review of A Large Clinical Series: Association of Cumulative Fluid Balance on Outcome in Acute Lung Injury: A Retrospective Review of the ARDSnet Tidal Volume Study Cohort. J Intensive Care Med 24:35-46.

4. Boyd JH, Forbes J, Nakada T-A, et al (2011) Fluid resuscitation in septic shock: a positive fluid balance and elevated central venous pressure are associated with increased mortality. Crit Care Med 39:259-265.

5. Hoste EA, Maitland K, Brudney CS, et al (2014) Four phases of intravenous fluid therapy: a conceptual model. Br J Anaesth 113:740-747.

6. Malbrain MLNG, Marik PE, Witters I, et al (2014) Fluid overload, de-resuscitation, and outcomes in critically ill or injured patients: a systematic review with suggestions for clinical practice. Anaesthesiol Intensive Ther 46:361-380.

7. Silversides JA, Ferguson AJ, McAuley DF, et al (2015) Fluid strategies and outcomes in patients with acute respiratory distress syndrome, systemic inflammatory response syndrome and sepsis: a protocol for a systematic review and meta-analysis. Syst Rev 4:162 
8. Higgins JPT, Green S, editors. (2011) The Cochrane Handbook for Systematic Reviews of Interventions, Version 5.1.0. The Cochrane Collaboration. http://handbook.cochrane.org/.

9. Moher D, Liberati A, Tetzlaff J, et al (2009) Preferred reporting items for systematic reviews and meta-analyses: the PRISMA statement. Ann Intern Med 151:264-270.

10. Wells GA, Shea B, O'Connell D, et al (2016) The Newcastle-Ottawa Scale (NOS) for assessing the quality of nonrandomised studies in meta-analyses. Ottawa Hopsital Research Institute. http://www.ohri.ca/programs/clinical_epidemiology/oxford.asp. Accessed 29 Apr 2016

11. Wan X, Wang W, Liu J, Tong T (2014) Estimating the sample mean and standard deviation from the sample size, median, range and/or interquartile range. BMC Med Res Methodol 14:135.

12. Guyatt $\mathrm{GH}$, Oxman AD, Vist GE, et al (2008) GRADE: an emerging consensus on rating quality of evidence and strength of recommendations. BMJ 336:924-926.

13. Mikkelsen ME, Christie JD, Lanken PN, et al (2012) The adult respiratory distress syndrome cognitive outcomes study: long-term neuropsychological function in survivors of acute lung injury. Am J Respir Crit Care Med 185:1307-1315.

14. Liu KD, Thompson BT, Ancukiewicz M, et al (2011) Acute kidney injury in patients with acute lung injury: Impact of fluid accumulation on classification of acute kidney injury and associated outcomes. Crit Care Med 39:2665-2671.

15. Grams ME, Estrella MM, Coresh J, et al (2011) Fluid Balance, Diuretic Use, and Mortality in Acute Kidney Injury. Clin J Am Soc Nephrol 6:966-973.

16. National Heart, Lung, and Blood Institute Acute Respiratory Distress Syndrome (ARDS) Clinical Trials Network, Wiedemann HP, Wheeler AP, et al (2006) Comparison of two fluid-management strategies in acute lung injury. N Engl J Med 354:2564-2575.

17. Martin GS, Moss M, Wheeler AP, et al (2005) A randomized, controlled trial of furosemide with or without albumin in hypoproteinemic patients with acute lung injury. Crit Care Med 33:1681-1687.

18. Martin GS, Mangialardi RJ, Wheeler AP, et al (2002) Albumin and furosemide therapy in hypoproteinemic patients with acute lung injury. Crit Care Med 30:2175-2182.

19. Chen C, Kollef MH (2015) Targeted Fluid Minimization Following Initial Resuscitation in Septic Shock: A Pilot Study. Chest 148:1462-1469.

20. Mitchell JP, Schuller D, Calandrino FS, Schuster DP (1992) Improved outcome based on fluid management in critically ill patients requiring pulmonary artery catheterization. Am Rev Respir Dis 145:990-998.

21. Hu W, Lin C-W, Liu B-W, et al (2014) Extravascular lung water and pulmonary arterial wedge pressure for fluid management in patients with acute respiratory distress syndrome. Multidiscip Resp Med 9:3.

22. Wang L, Long X, Lv M (2014) Effect of different liquid management strategies on the prognosis of acute respiratory distress syndrome. Journal of the Dalian Medical University 36:140-143.

23. Zhang Z, Ni H, Qian Z (2015) Effectiveness of treatment based on PiCCO parameters in critically ill patients with septic shock and/or acute respiratory distress syndrome: a randomized controlled trial. Intensive Care Med 41:444-451.

24. Richard J-C, Bayle F, Bourdin G, et al (2015) Preload dependence indices to titrate volume expansion during septic shock: a randomized controlled trial. Crit Care 19:5.

25. Benakatti G, Singhi S, Muralidharan J, A B (2012) Conventional vs. restrictive maintenance fluid regime in children with septic shock after initial resuscitation: A randomized open label controlled 
trial. Arch Dis Child 97:Suppl2 A5.

26. Hjortrup PB, Haase N, Bundgaard H, et al (In press) Restricting volumes of resuscitation fluid in septic shock after the initial management - the CLASSIC randomised, parallel-group, multicentre feasibility trial. Intensive Care Med.

27. Abulebda K, Cvijanovich NZ, Thomas NJ, et al (2014) Post-ICU Admission Fluid Balance and Pediatric Septic Shock Outcomes. Crit Care Med 42:397-403.

28. Acheampong A, Vincent JL (2015) A positive fluid balance is an independent prognostic factor in patients with sepsis. Crit Care 19:251.

29. Bhaskar P, Dhar AV, Thompson M, et al (2015) Early fluid accumulation in children with shock and ICU mortality: a matched case-control study. Intensive Care Med 41:1445-1453.

30. Botdorf J, Kashyap R, O'Horo JC, et al (2015) Retrospective Analysis of Fluid Balance in the First 48 Hours of Sepsis and Outcome. Am J Respir Crit Care Med 191:A4497.

31. Chen Q-H, Zheng R-Q, Lin $\mathrm{H}$, et al (2011) The impact of different fluid management on mortality in patients with septic shock. Zhongguo Wei Zhong Bing Ji Jiu Yi Xue 23:142-145.

32. Cordemans C, De laet I, Van Regenmortel N, et al (2012) Aiming for a negative fluid balance in patients with acute lung injury and increased intra- abdominal pressure: a pilot study looking at the effects of PAL-treatment. Ann Intensive Care 2:S15.

33. De Oliveira FSV, Freitas FGR, Ferreira N, et al (2015) Journal of Critical Care. J Crit Care 30:97101.

34. Fiorenza M, Pass S (2013) Outcomes Associated with Maintaining Central Venous Pressure Target in Severe Sepsis \& Septic Shock. Crit Care Med 41:A270.

35. Flori HR, Church G, Liu KD, et al (2011) Positive fluid balance is associated with higher mortality and prolonged mechanical ventilation in pediatric patients with acute lung injury. Crit Care Res Pract 2011:854142.

36. Herrera Gutierrez M, Arias-Verdu D, Lozano-Saez R, Barruecos G (2013) Fluids management in kidney dysfunction and mortality in septic patients with special emphasis in the role of administration of hydroxyethyl starch. Intensive Care Med 39:703.

37. Koonrangsesomboon W, Khwannimit B (2015) Impact of positive fluid balance on mortality and length of stay in septic shock patients. Indian J Crit Care Med 19:708.

38. Micek ST, McEvoy C, McKenzie M, et al (2013) Fluid balance and cardiac function in septic shock as predictors of hospital mortality. Crit Care 17:R246.

39. Murai A, Ishikura $\mathrm{H}$, Matsumoto $\mathrm{N}$, et al (2014) Impact of fluid management during the three ICU days after admission in patients with ARDS. Crit Care 18:P25.

40. Murphy CV, Schramm GE, Doherty JA, et al (2009) The Importance of Fluid Management in Acute Lung Injury Secondary to Septic Shock. Chest 136:102-109.

41. Perez-Fernandez XL, Sabater J, Koborzan MR, et al (2011) Fluid balance on early stages of septic shock patients with continuous renal replacement techniques. Intensive Care Med 35:970.

42. Raimundo M, Ferreira N, Marques A, et al (2012) Impact of fluid balance in the outcome of septic critically ill patients. Intensive Care Med 38:696.

43. Simmons RS, Berdine GG, Seidenfeld JJ, et al (1987) Fluid balance and the adult respiratory distress syndrome. Am Rev Respir Dis 135:924-929. 
44. Udeozo OI, Selby MG, Cartin-Ceba R, et al (2009) Early but not late fluid resuscitation was associated with improved outcome in septic shock. Blood Purif 27:282.

45. Vincent JL, Sakr Y, Sprung CL, et al (2006) Sepsis in European intensive care units: results of the SOAP study. Crit Care Med 34:344-353.

46. Sakr Y, Vincent JL, Reinhart K, et al (2005) High tidal volume and positive fluid balance are associated with worse outcome in acute lung injury. Chest 128:3098-3108.

47. Wang H, Cui N, Su L, et al (2016) Prognostic value of extravascular lung water and its potential role in guiding fluid therapy in septic shock after initial resuscitation. J Crit Care. 33:106-113.

48. Wilkowski A, Goeckenjan G (1988) Effect of fluid withdrawal on pulmonary course and prognosis of acute lung failure. Intensivmed Notfmed 25:10-16.

49. Willson DF, Thomas NJ, Tamburro R, et al (2015) The relationship of fluid administration to outcome in the pediatric calfactant in acute respiratory distress syndrome trial. Pediatr Crit Care Med 14:666672.

50. Yao B, Liu D, Wang X, Zhang H (2014) Negative fluid balance predicts survival in patients with septic shock. Zhonghua Yi Xue Za Zhi 94:3206-3210.

51. Zhang Z, Zhang Z, Xue Y, et al (2012) Prognostic value of B-type natriuretic peptide (BNP) and its potential role in guiding fluid therapy in critically ill septic patients. ScandJ Trauma Resusc Emerg Med 20:1-1.

52. Zhang X-Q, Tian H-H, Geng H-M, et al (2013) The influence of volume load on prognosis of patients with sepsis induced acute kidney injury. Zhonghua Wei Zhong Bing Ji Jiu Yi Xue 25:411-414.

53. Wang H, Cui N, Su L, et al (2016) Prognostic value of extravascular lung water and its potential role in guiding fluid therapy in septic shock after initial resuscitation. J Crit Care 33:106-113.

54. Valentine SL, Sapru A, Higgerson RA, et al (2012) Fluid balance in critically ill children with acute lung injury. Crit Care Med 40:2883-2889.

55. Rodriguez A, De Haro C, Teixido C, et al (2013) Fluid balance in patients with severe sepsis and/or septic shock. Intensive Care Med 39:14.

56. Cronhjort M, Hjortrup PB, Holst LB, et al (2016) Association between fluid balance and mortality in patients with septic shock: a post hoc analysis of the TRISS trial. Acta Anaesthesiol Scand 60:925933.

57. Sun Z, Sun F, Niu C, et al (2015) Continuous renal replacement therapy and negative fluid balance improves renal function and prognosis of patients with acute kidney injury in sepsis. Zhonghua Wei Zhong Bing Ji Jiu Yi Xue 27:321-326.

58. Spicer A, Lo V, Khemani RG, et al (2014) Fluid overload is associated with mortality in paediatric acute respiratory distress syndrome (ARDS) only in the setting of acute kidney injury (AKI). Arch Dis Child 99:A160-A161.

59. Saito N, Yagi T, Hara Y, et al (2012) Implementation of an optimal fluid management protocol using the PiCCO system delays development of ARDS secondary to severe sepsis. Crit Care 16:P243.

60. Grissom CK, Hirshberg EL, Dickerson JB, et al (2015) Fluid management with a simplified conservative protocol for the acute respiratory distress syndrome. Crit Care Med 43:288-295.

61. Smith SH, Perner A (2012) Higher vs. lower fluid volume for septic shock:clinical characteristics and outcome in unselectedpatients in a prospective, multicenter cohort. Crit Care 16:R76.

62. Kongsayreepong S, Nitikaroon P (2013) Early acute kidney injury in postoperative severe sepsis/ 
septic shock septic admitting to general surgical intensive care unit. Intensive Care Med 39:0181.

63. Dellinger RP, Levy MM, Rhodes A, et al (2013) Surviving Sepsis Campaign: international guidelines for management of severe sepsis and septic shock, 2012. Intensive Care Med 39:165-228.

64. McArdle GT, McAuley DF, McKinley A, et al (2009) Preliminary results of a prospective randomized trial of restrictive versus standard fluid regime in elective open abdominal aortic aneurysm repair. Ann Surg 250:28-34.

65. Pandharipande PP, Girard TD, Jackson JC, et al (2013) Long-term cognitive impairment after critical illness. N Engl J Med 369:1306-1316.

66. Blackwood B, Clarke M, McAuley DF, et al (2014) How outcomes are defined in clinical trials of mechanically ventilated adults and children. Am J Respir Crit Care Med 189:886-893.

67. Roth D, Heidinger B, Havel C, Herkner H (2016) Different Mortality Time Points in Critical Care Trials: Current Practice and Influence on Effect Estimates in Meta-Analyses. Crit Care Med 44:e737741

68. Lu J, Goh SJ, Tng PYL, et al (2009) Systemic inflammatory response following acute traumatic brain injury. Front Biosci 14:3795-3813.

69. Lord JM, Midwinter MJ, Chen Y-F, et al (2014) The systemic immune response to trauma: an overview of pathophysiology and treatment. Lancet 384:1455-1465.

70. Bernard GR, Artigas A, Brigham KL, et al (1994) The American-European Consensus Conference on ARDS. Definitions, mechanisms, relevant outcomes, and clinical trial coordination. In: Am. J. Respir. Crit. Care Med 149:818-824

71. ARDS Definition Task Force, Ranieri VM, Rubenfeld GD, et al (2012) Acute respiratory distress syndrome: the Berlin Definition. JAMA 307:2526-2533.

72. Bone RC, Balk RA, Cerra FB, et al (1992) Definitions for sepsis and organ failure and guidelines for the use of innovative therapies in sepsis. The ACCP/SCCM Consensus Conference Committee. American College of Chest Physicians/Society of Critical Care Medicine. Chest 101:1644-1655. 
Figure 1. Study flow diagram. *Some studies had multiple reasons for exclusion.

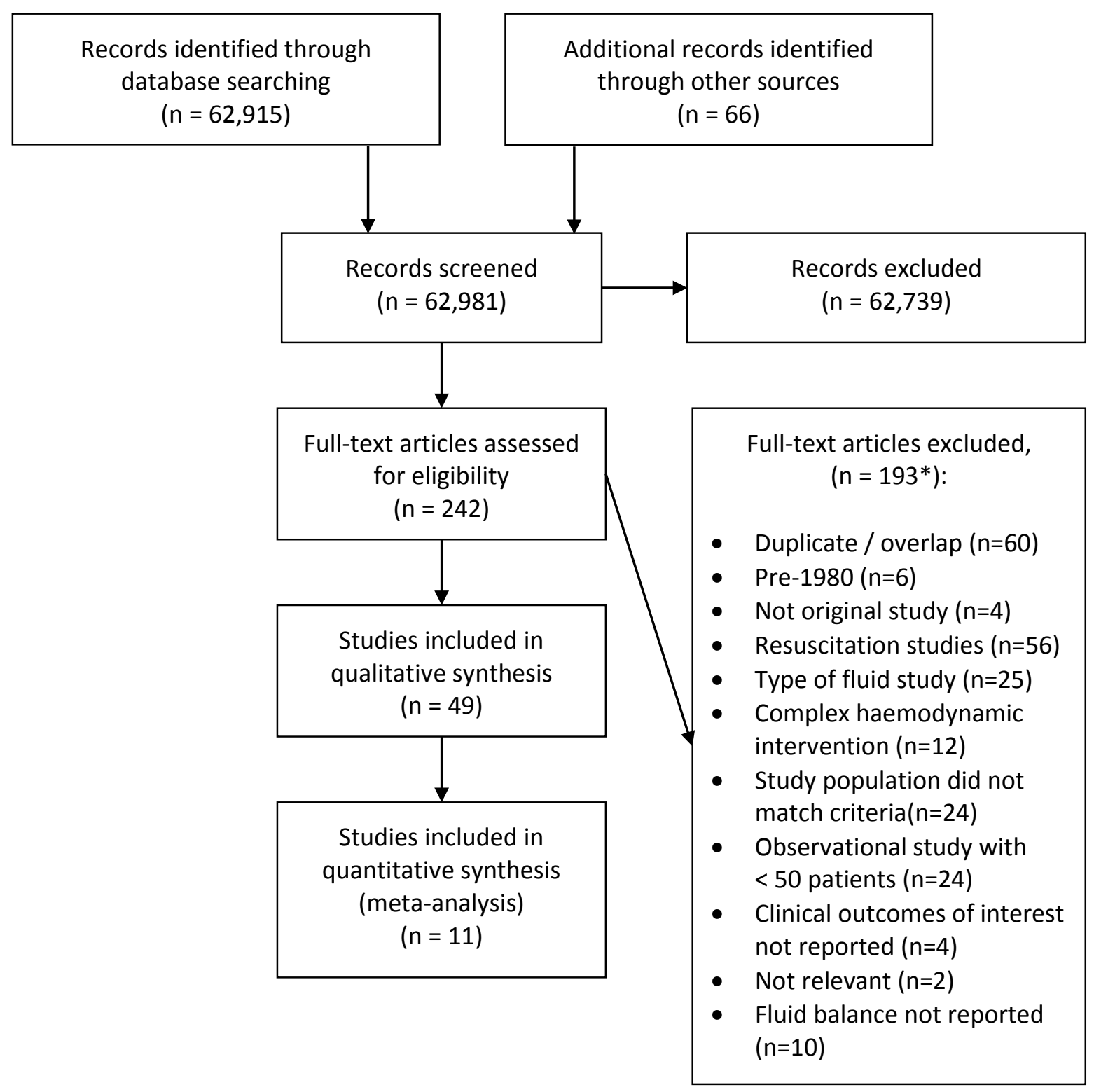


Figure 2. Risk of bias assessment for randomised trials.

\begin{tabular}{|c|c|c|c|c|c|c|c|}
\hline & 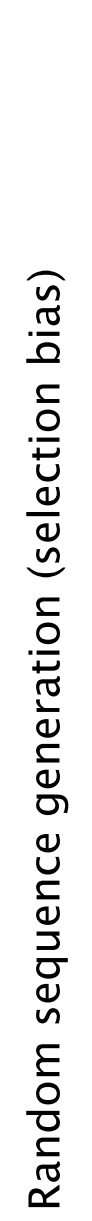 & 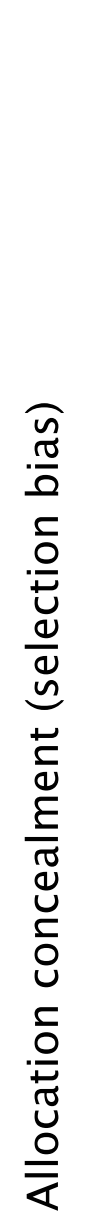 & 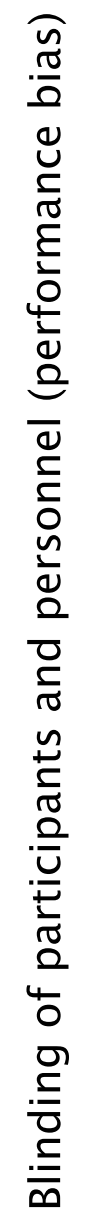 & 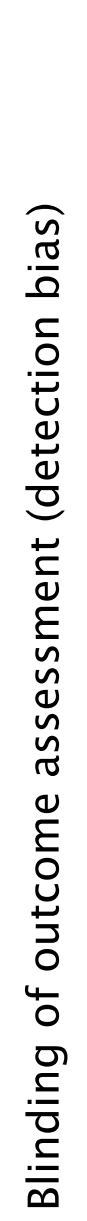 & 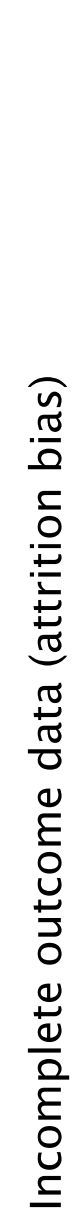 & 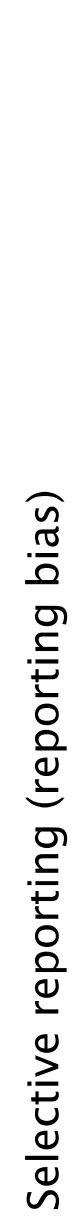 & $\begin{array}{l}\frac{\tilde{\sigma}}{0} \\
\frac{1}{0} \\
\frac{1}{5} \\
0\end{array}$ \\
\hline Benakatti et al. 2014 & $?$ & $?$ & $?$ & $?$ & $?$ & $?$ & $?$ \\
\hline Chen and Kollef. 2015 & $?$ & $?$ & - & + & + & + & + \\
\hline Hjortrup et al. 2016 & + & + & + & + & + & + & + \\
\hline Hu et al. 2014 & $?$ & $?$ & - & $?$ & + & $?$ & + \\
\hline Martin et al. 2002 & + & + & + & + & + & $?$ & + \\
\hline Martin et al. 2005 & + & + & + & + & + & + & + \\
\hline Mitchell et al. 1992 & $?$ & $?$ & + & + & $?$ & $?$ & + \\
\hline Richard et al. 2015 & + & + & + & + & + & + & + \\
\hline Wang et al. 2014 & $?$ & $?$ & $?$ & $?$ & + & $?$ & $?$ \\
\hline Wiedemann et al. 2006 & + & + & + & + & + & + & + \\
\hline Zhang et al. 2015 & + & + & + & + & + & + & + \\
\hline
\end{tabular}


Figure 3. Forest plot for mortality at most protracted time point available, conservative or deresuscitative fluid strategy versus standard care or liberal fluid strategy.

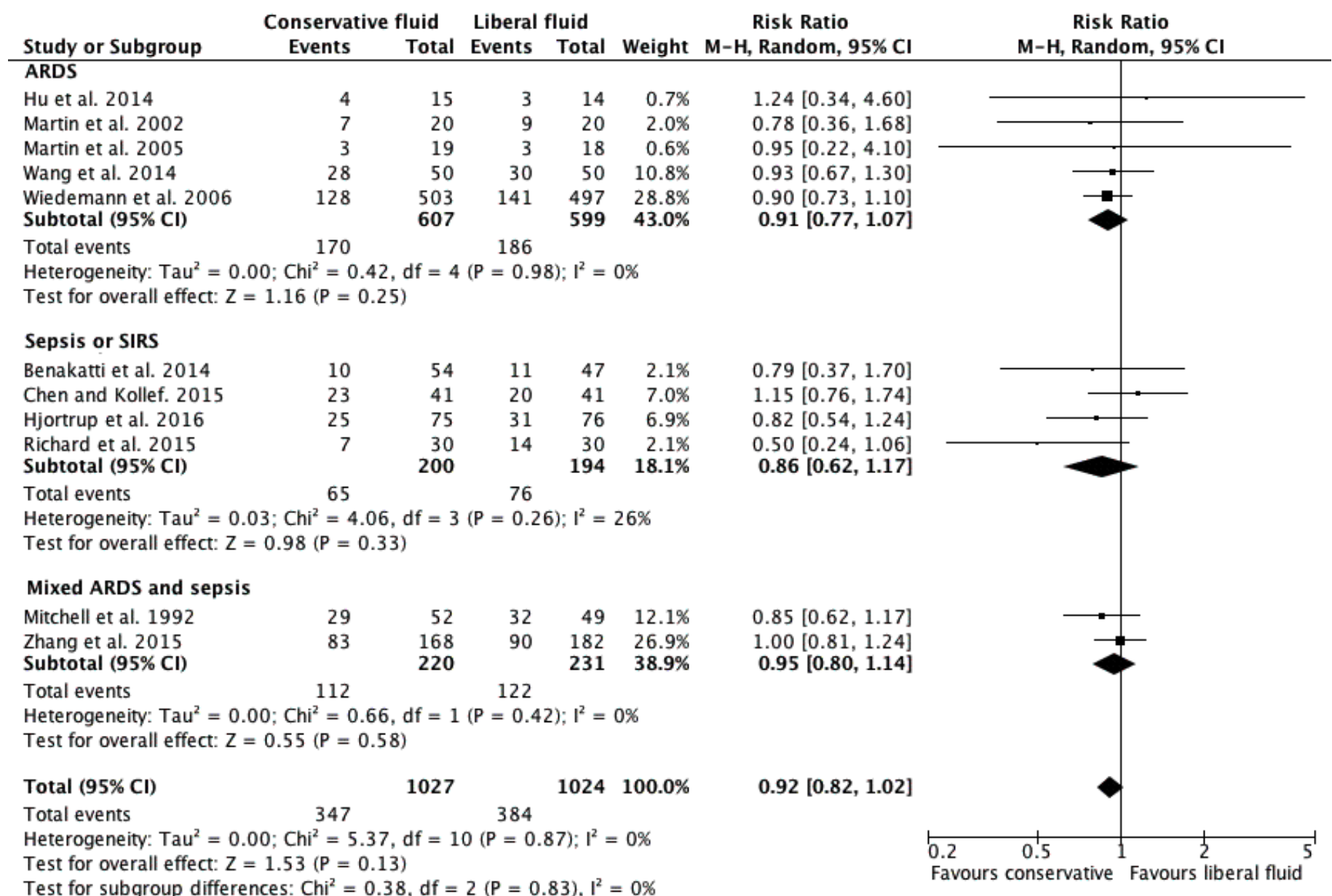


Figure 4. Forest plot for outcome of ventilator-free days.

Conservative fluid Liberal fluid

Mean Difference

Study or Subgroup $\quad$ Mean [Days] SD [Days] Total Mean [Days] SD [Days] Total Weight

Chen and Kollef. 2015

5.5
9
21.4
10.3
14.6
12.7
15.8

$\begin{array}{rr}9.4 & 41 \\ 17.9 & 168\end{array}$

7.4
10.3

Hjortrup et al. 2016

Martin et al. 2005

Wiedemann et al. 2006

Richard et al. 2015

Benakatti et al. 2014

$\begin{array}{rr}9.7 & 75 \\ 8 & 20 \\ 11.2 & 503 \\ 18.7 & 30 \\ 10.8 & 54\end{array}$

7.4
10.3
19.8

19.8

8

Total (95\% Cl)

891

Heterogeneity: $\mathrm{Tau}^{2}=0.33 ; \mathrm{Chi}^{2}=6.63, \mathrm{df}=6(\mathrm{P}=0.36) ; \mathrm{I}^{2}=9 \%$

Test for overall effect: $Z=2.78(P=0.005)$

$\begin{array}{rrr}12.9 & 41 & 6.5 \% \\ 18.7 & 182 & 10.3 \% \\ 11.1 & 76 & 13.3 \% \\ 8 & 20 & 6.4 \% \\ 11.1 & 497 & 51.6 \% \\ 16.3 & 30 & 2.1 \% \\ 9.4 & 47 & 9.8 \%\end{array}$

$893 \quad 100.0 \%$ IV, Random, 95\% Cl [Days]

$3 \%$

$3 \%$

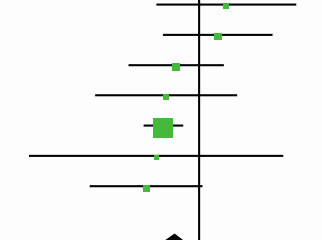

Favours conservative Favours liberal 
Figure 5. Forest plot for ICU length of stay, conservative or deresuscitative fluid strategy versus standard care or liberal fluid strategy.

Conservative fluid

Liberal fluid

Mean Difference

Mean Difference

Study or Subgroup Mean [Days] SD [Days] Total Mean [Days] SD [Day

\section{Benakatti et al. 2014}

$\begin{array}{lrrr}7.1 & 5.5 & 54 & 10.3\end{array}$

Hjortrup et al. 2016

Hu et al. 2014

$\begin{array}{lll}7.7 & 6.1 & 75\end{array}$

Mitchell et al. 1992

Richard et al. 2015

Wang et al. 2014

12.5

$3.5 \quad 15$

10.3
6

[Days] Total

\begin{tabular}{rrr}
.5 & 47 & $15.5 \%$ \\
\hline 3 & 76 & $17.5 \%$
\end{tabular}

$\begin{array}{ll}47 & 15.5 \% \\ 76 & 17.5 \%\end{array}$

IV, Random, 95\% Cl [Days]

Zhang et al. 2015

18.7

12.1

$10.7 \quad 52$

$\begin{array}{rr}17.1 & 30 \\ 3.2 & 50\end{array}$

15.5

$\begin{array}{lll}2.5 & 14 & 16.1 \%\end{array}$

$\begin{array}{lll}10.7 & 49 & 9.8 \%\end{array}$

$14.8 \quad 30 \quad 3.9 \%$

$\begin{array}{lll}4.6 & 50 & 18.5 \%\end{array}$

Total $(95 \% \mathrm{Cl})$

444

$8.2 \quad 182 \quad 18.7 \%$

Heterogeneity: $\mathrm{Tau}^{2}=3.74 ; \mathrm{Chi}^{2}=24.47, \mathrm{df}=6(\mathrm{P}=0.0004) ; \mathrm{I}^{2}=75 \%$

$448 \quad 100.0 \%$

$-3.20[-5.57,-0.83]$

$0.70[-1.12,2.52]$

$-3.00[-5.20,-0.80]$

$-4.50[-8.68,-0.32]$

$1.70[-6.39,9.79]$

$-3.70[-5.25,-2.15]$

$0.20[-1.30,1.70]$

Test for overall effect: $Z=2.09(P=0.04)$

$-1.88[-3.64,-0.12]$

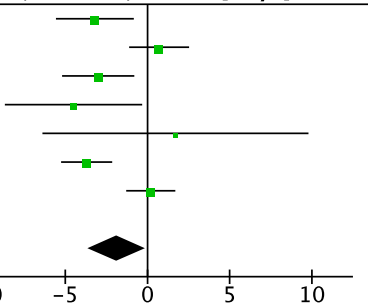

Favours conservative Favours liberal 


\begin{tabular}{|c|c|c|c|c|c|}
\hline $\begin{array}{l}\text { Author and } \\
\text { publication } \\
\text { year }\end{array}$ & $\begin{array}{l}\text { Methods } \\
\text { and Setting }\end{array}$ & Participants & $\begin{array}{l}\text { Summary of conservative or } \\
\text { deresuscitative fluid strategy }\end{array}$ & $\begin{array}{l}\text { Summary of liberal fluid strategy } \\
\text { or usual care }\end{array}$ & Key Outcomes \\
\hline $\begin{array}{l}\text { Mitchell et al, } \\
1992\end{array}$ & $\begin{array}{l}\text { RCT } \\
\text { Single } \\
\text { academic } \\
\text { centre in } \\
\text { United } \\
\text { States }\end{array}$ & $\begin{array}{l}\mathrm{n}=101 \\
\text { Inclusion criteria: } \\
\text {-Admitted to medical ICU } \\
\text {-Pulmonary artery } \\
\text { catheter inserted } \\
\text { Exclusion criteria: } \\
\text {-Technical reasons } \\
\text {-Logistical reasons } \\
\text {-Allergy to iodine dye } \\
\text {-Pregnancy or lactation }\end{array}$ & $\begin{array}{l}\text {-Extra-Vascular Lung Water } \\
\text { (EVLW)-guided strategy. } \\
\text { Restriction of fluid intake when } \\
\text { ELWW } \geq 7 \mathrm{ml} / \mathrm{kg} \text { and diuresis if } \\
\text { stable. } \\
\text {-Mean fluid balance was } 142+/ \text { - } \\
3632 \mathrm{ml} \text { at } 60 \text { hours* } \\
\text {-Mean daily fluid balance over } \\
\text { study period: } 0.8 \mathrm{ml} / \mathrm{kg} / \text { day }\end{array}$ & $\begin{array}{l}\text {-Pulmonary capillary wedge } \\
\text { pressure (PCWP) - guided } \\
\text { strategy with target range of 10- } \\
17 \mathrm{mmHg} \text {. } \\
\text {-Mean fluid balance was } 2239 \\
\text { +/-3695 } \mathrm{ml} \text { at } 47 \text { hours* } \\
\text {-Mean daily fluid balance over } \\
\text { study period: } 16.3 \mathrm{ml} / \mathrm{kg} / \text { day }\end{array}$ & $\begin{array}{l}\text {-ICU mortality } \\
\text {-Hospital mortality } \\
\text {-Duration of } \\
\text { mechanical } \\
\text { ventilation } \\
\text {-Length of ICU stay }\end{array}$ \\
\hline $\begin{array}{l}\text { Martin et al, } \\
2002\end{array}$ & $\begin{array}{l}\text { RCT } \\
\text { Two } \\
\text { academic } \\
\text { centres in } \\
\text { United } \\
\text { States }\end{array}$ & $\begin{array}{l}\mathrm{n}=37 \\
\text { Inclusion criteria: } \\
\text {-ARDS } \\
\text {-Serum total protein } \leq \\
5 \mathrm{~g} / \mathrm{dL} \\
\text {-Ongoing nutritional } \\
\text { support } \\
\text {-Mechanical ventilation } \geq \\
48 \text { hours } \\
\text { Exclusion criteria: } \\
\text {-Haemodynamic instability } \\
\text {-Renal disease } \\
\text {-Hepatic failure or } \\
\text { cirrhosis } \\
\text {-Age }<8 \text { or }>80 \text { years } \\
\text {-Pregnancy } \\
\text {-Serum sodium }>150 \\
\text { mmol/L or potassium }<2.5 \\
\text { mmol/L }\end{array}$ & $\begin{array}{l}\text {-Furosemide infusion titrated to } \\
\text { weight loss of } \geq 1 \mathrm{~kg} / \mathrm{day}, \text { and } \\
25 \mathrm{~g} \text { IV albumin } 8 \text { hourly for } 5 \\
\text { days } \\
\text {-Mean weight loss of } 10.0 \mathrm{~kg} \\
\text { after } 5 \text { days* } \\
\text {-Mean daily fluid balance over } \\
\text { study period: }-47.6 \mathrm{ml} / \mathrm{kg} / \text { day }\end{array}$ & $\begin{array}{l}\text {-Dual placebo } \\
\text {-Mean weight loss of } 4.7 \mathrm{~kg} \text { after } \\
5 \text { days* } \\
\text {-Mean daily fluid balance over } \\
\text { study period: }-22.4 \mathrm{ml} / \mathrm{kg} / \text { day }\end{array}$ & $\begin{array}{l}\text {-30 day mortality } \\
\text {-ICU-free days } \\
\text {-Ventilator-free days } \\
\text {-Length of hospital } \\
\text { stay }\end{array}$ \\
\hline Martin et al, & $\mathrm{RCT}$ & $n=40$ & -Furosemide $20 \mathrm{mg}$ IV bolus & -Furosemide $20 \mathrm{mg}$ IV bolus & -30 day mortality \\
\hline
\end{tabular}




\begin{tabular}{|c|c|c|c|c|c|}
\hline 2005 & $\begin{array}{l}\text { Two } \\
\text { academic } \\
\text { centres in } \\
\text { United } \\
\text { States }\end{array}$ & $\begin{array}{l}\text { Inclusion criteria: } \\
\text {-ARDS } \\
\text {-Serum total protein }<6 \\
\text { g/dL } \\
\text { Exclusion criteria: } \\
\text {-Haemodynamic instability } \\
\text {-Renal disease or } \\
\text { cirrhosis } \\
\text {-Age }<18 \text { years } \\
\text {-Pregnancy } \\
\text {-Serum sodium }>155 \\
\text { mmol/L or potassium }< \\
2.5 \text { mmol/L }\end{array}$ & $\begin{array}{l}\text { followed by infusion, and } 25 \mathrm{~g} \text { IV } \\
\text { albumin } 8 \text { hourly for } 3 \text { days } \\
\text {-Mean net fluid balance after } 3 \\
\text { days was }-5480 \mathrm{ml}^{*} \\
\text {-Mean daily fluid balance over } \\
\text { study period: }-15.7 \mathrm{ml} / \mathrm{kg} / \text { day }\end{array}$ & $\begin{array}{l}\text { followed by infusion, with } 0.9 \% \\
\text { saline placebo for } 3 \text { days. } \\
\text {-Mean net fluid balance at } 3 \\
\text { days was }-1490 \mathrm{ml}^{\star} \\
\text {-Mean daily fluid balance over } \\
\text { study period: }-4.3 \mathrm{ml} / \mathrm{kg} / \text { day }\end{array}$ & $\begin{array}{l}\text {-Ventilator-free days } \\
\text {-Change in SOFA } \\
\text { scores }\end{array}$ \\
\hline $\begin{array}{l}\text { Wiedemann et } \\
\text { al, } 2006\end{array}$ & $\begin{array}{l}\text { RCT } \\
\text { Multiple } \\
\text { community } \\
\text { and } \\
\text { academic } \\
\text { ICUs in } \\
\text { United } \\
\text { States and } \\
\text { Canada }\end{array}$ & $\begin{array}{l}\text { n=1000 } \\
\text { Inclusion criteria: } \\
\text {-ARDS } \\
\text {-Intubated and } \\
\text { mechanically ventilated } \\
\text {-Presence or intention to } \\
\text { insert a central venous } \\
\text { catheter } \\
\text { Exclusion criteria: } \\
\text {-Presence of ALI/ARDS } \\
\text { for > } 48 \text { hours } \\
\text {-Severe chronic illness } \\
\text { likely to independently } \\
\text { influence survival } \\
\text {-Irreversible terminal } \\
\text { illness }\end{array}$ & $\begin{array}{l}\text {-Complex algorithm with fluid } \\
\text { boluses or diuretics } \\
\text { administered as directed by } \\
\text { filling pressures (CVP or } \\
\text { PCWP). } \\
\text {-41\% of protocol instructions } \\
\text { involved administration of } \\
\text { furosemide, } 6 \% \text { involved fluid } \\
\text { boluses } \\
\text {-At } 7 \text { days, net fluid balance was } \\
\text {-136 ml +/- } 11012 \text { ml* } \\
\text {-Mean daily fluid balance over } \\
\text { study period: }-0.3 \text { ml/kg/day }\end{array}$ & $\begin{array}{l}\text {-Complex algorithm with fluid } \\
\text { boluses or diuretics } \\
\text { administered as directed to } \\
\text { target higher filling pressures } \\
\text { (CVP or PCWP) than in } \\
\text { conservative group. } \\
\text {-10\% of protocol instructions } \\
\text { involved administration of } \\
\text { furosemide, } 15 \% \text { involved fluid } \\
\text { boluses } \\
\text {-At } 7 \text { days, net fluid balance was } \\
6992 \text { ml +/- } 11191 \text { ml* } \\
\text {-Mean daily fluid balance over } \\
\text { study period: } 14.3 \text { ml/kg/day }\end{array}$ & $\begin{array}{l}\text {-60-day mortality } \\
\text {-Ventilator-free days } \\
\text {-ICU-free days } \\
\text {-Renal failure-free } \\
\text { days } \\
\text {-RRT use } \\
\text {-CNS failure-free } \\
\text { days }\end{array}$ \\
\hline Hu et al, 2014 & $\begin{array}{l}\text { RCT } \\
\text { Single } \\
\text { centre in } \\
\text { China }\end{array}$ & $\begin{array}{l}\mathrm{n}=29 \\
\text { Inclusion criteria: } \\
\text {-ALI/ARDS (AECC } \\
\text { criteria) } \\
\text {-Admitted to ICU }\end{array}$ & $\begin{array}{l}\text {-Extravascular lung water target } \\
\text { value set at } 3-7 \mathrm{ml} / \mathrm{kg} \text {, using } \\
\text { diuretics or CRRT } \\
\text {-Fluid administration not }\end{array}$ & $\begin{array}{l}\text {-Pulmonary artery occlusion } \\
\text { pressure target of 8-12 mmHg, } \\
\text { using diuretics or CRRT } \\
\text {-Fluid administration not } \\
\text { protocolised }\end{array}$ & $\begin{array}{l}-60 \text { day mortality } \\
\text {-Duration of } \\
\text { mechanical } \\
\text { ventilation } \\
\text {-Length of ICU stay }\end{array}$ \\
\hline
\end{tabular}




\begin{tabular}{|c|c|c|c|c|c|}
\hline & & $\begin{array}{l}\text { Exclusion criteria: } \\
\text {-Pre-existing } \\
\text { comorbidities including } \\
\text { pulmonary hypertension, } \\
\text { pneumonectomy, and } \\
\text { interstitial lung disease }\end{array}$ & $\begin{array}{l}\text { protocolised } \\
\text {-Mean fluid balance at } 7 \text { days } \\
\text { was }-783 \mathrm{ml}+/-391 \mathrm{ml} \\
\text {-Estiamted mean daily fluid } \\
\text { balance over study period: }-1.6 \\
\text { ml/kg/day }\end{array}$ & $\begin{array}{l}\text {-Mean fluid balance at } 7 \text { days } \\
\text { was }-256 \mathrm{ml}+/-514 \mathrm{ml} \\
\text {-Estimated mean daily fluid } \\
\text { balance over study period: }-0.5 \\
\mathrm{ml} / \mathrm{kg} / \text { day }\end{array}$ & \\
\hline $\begin{array}{l}\text { Benakatti et } \\
\text { al, } 2014\end{array}$ & $\begin{array}{l}\text { RCT } \\
\text { Single } \\
\text { centre in } \\
\text { India }\end{array}$ & $\begin{array}{l}\mathrm{n}=101 \\
\text { Inclusion criteria: } \\
\text {-Children aged 3-144 } \\
\text { months } \\
\text {-Septic shock following } \\
\text { fluid resuscitation } \\
\text { Exclusion criteria: } \\
\text {-None reported }\end{array}$ & $\begin{array}{l}\text {-Maintenance fluid administered } \\
\text { at } 80 \% \text { of calculated required } \\
\text { rate } \\
\text {-At } 10 \text { days, mean net fluid } \\
\text { balance was }-42.6 \mathrm{ml} / \mathrm{kg}+/-82.6 \\
\mathrm{ml} / \mathrm{kg}^{*} \\
\text {-Mean daily fluid balance over } \\
\text { study period: }-33.9 \mathrm{ml} / \mathrm{kg} / \text { day }\end{array}$ & $\begin{array}{l}\text {-Regimen not clearly reported } \\
\text {-At } 10 \text { days, net fluid balance } \\
\text { was } 339 \mathrm{ml} / \mathrm{kg}+/-117 \mathrm{ml} / \mathrm{kg}^{*} \\
\text {-Mean daily fluid balance over } \\
\text { study period: }-4.26 \mathrm{ml} / \mathrm{kg} / \text { day }\end{array}$ & $\begin{array}{l}\text {-28 day mortality } \\
\text {-Ventilator-free days } \\
\text {-Length of ICU stay }\end{array}$ \\
\hline $\begin{array}{l}\text { Wang et al, } \\
2014\end{array}$ & $\begin{array}{l}\text { RCT } \\
\text { Single } \\
\text { centre in } \\
\text { China }\end{array}$ & $\begin{array}{l}\text { n=100 } \\
\text { Inclusion criteria: } \\
\text {-ARDS (AECC definition) } \\
\text { Exclusion criteria: } \\
\text {-Age < } 13 \text { years } \\
\text {-Contraindication to } \\
\text { central venous catheter } \\
\text {-ARDS criteria met for > } \\
48 \text { hours pre-enrollment } \\
\text {-Myocardial infarction in } \\
\text { last } 30 \text { days } \\
\text {-History of COPD or } \\
\text { neuromuscular disorder } \\
\text { affecting respiration }\end{array}$ & $\begin{array}{l}\text {-Extravascular lung water index } \\
\text { target of } 3-7 \mathrm{ml} / \mathrm{kg} \text {. Regimen } \\
\text { used not clearly reported } \\
\text {-At } 7 \text { days, mean net fluid } \\
\text { balance was }-9.6 \mathrm{ml}^{*} \\
\text {-Estiamted mean daily fluid } \\
\text { balance over study period: }-0.02 \\
\text { ml/kg/day }\end{array}$ & $\begin{array}{l}\text {-Regimen used not clearly } \\
\text { reported } \\
\text {-At } 7 \text { days, mean net fluid } \\
\text { balance was } 7083.6 \mathrm{ml}^{*} \\
\text {-Estiamted mean daily fluid } \\
\text { balance over study period: } 14.5 \\
\text { ml/kg/day }\end{array}$ & $\begin{array}{l}\text {-60 day mortality } \\
\text {-Duration of } \\
\text { mechanical } \\
\text { ventilation } \\
\text {-Length of ICU stay } \\
\text {-Cognitive function } \\
\text { domain of QLQ-C30 } \\
\text { quality of life score }\end{array}$ \\
\hline $\begin{array}{l}\text { Chen and } \\
\text { Kollef, } 2015\end{array}$ & RCT & $n=82$ & $\begin{array}{l}\text {-Targeted fluid minimisation } \\
\text { comprising: fluid-responsiveness }\end{array}$ & -Usual care & $\begin{array}{l}\text {-Hospital mortality } \\
\text {-Ventilator-free days }\end{array}$ \\
\hline
\end{tabular}




\begin{tabular}{|c|c|c|c|c|c|}
\hline & $\begin{array}{l}\text { Single } \\
\text { academic } \\
\text { centre in } \\
\text { United } \\
\text { States }\end{array}$ & $\begin{array}{l}\text { Inclusion criteria: } \\
\text {-Hypotension due to } \\
\text { septic shock } \\
\text {-Requirement for } \geq 12 \\
\text { hours of vasoactive drugs } \\
\text { to treat hypotension after } \\
\text { fluid resuscitation } \geq 30 \\
\text { ml/kg IV fluid } \\
\text { Exclusion criteria: } \\
\text {-Age <18 years } \\
\text {-Pre-existing end stage } \\
\text { renal disease } \\
\text {-Pregnancy } \\
\text {-Comfort-only goals of } \\
\text { care }\end{array}$ & $\begin{array}{l}\text { testing before fluid } \\
\text { administration, concentration of } \\
\text { drug infusions, discontinuation } \\
\text { of maintenance fluids } \\
\text {-Diuretics and ultrafiltration not } \\
\text { protocolised } \\
\text {-At } 5 \text { days, median net fluid } \\
\text { balance was } 2641 \text { ml (IQR - } \\
\text { 1837-5075) } \\
\text {-Estimated mean daily fluid } \\
\text { balance over study period: } 7.5 \\
\text { ml/kg/day }\end{array}$ & $\begin{array}{l}\text {-At } 5 \text { days, median net fluid } \\
\text { balance was } 3616 \text { ml (IQR - } \\
\text { 1513-9746 ml) } \\
\text {-Estiamted mean daily fluid } \\
\text { balance over study period: } 10.3 \\
\text { ml/kg/day }\end{array}$ & -RRT use \\
\hline $\begin{array}{l}\text { Zhang et al, } \\
2015\end{array}$ & $\begin{array}{l}\text { RCT } \\
\text { Two tertiary } \\
\text { centres in } \\
\text { China }\end{array}$ & $\begin{array}{l}\text { n=350 } \\
\text { Inclusion criteria: } \\
\text {-Septic shock or ARDS } \\
\text { (Berlin definition) } \\
\text {-<24hours since ICU } \\
\text { admission } \\
\text { Exclusion criteria: } \\
\text {-Age <18 years } \\
\text { - Haemorrhagic shock } \\
\text { - Moribund state } \\
\text { - Absence of informed } \\
\text { consent } \\
\text { - Contra-indication to } \\
\text { catheter insertion } \\
\text { - Conditions likely to } \\
\text { render PiCCO } \\
\text { innaccurate }\end{array}$ & $\begin{array}{l}\text {-Fluid boluses targeted to } \\
\text { intrathoracic blood volume index } \\
\text { (ITBVI) } 850-1000 \mathrm{ml} / \mathrm{m}^{2} \\
\text {-Identical algorithm for } \\
\text { noradrenaline, dobutamine and } \\
\text { nitrate use in both groups } \\
\text {-At } 7 \text { days, mean net fluid } \\
\text { balance was } 3821.6 \mathrm{ml} \\
\text {-Estimated mean daily fluid } \\
\text { balance over study period: } 7.8 \\
\text { ml/kg/day }\end{array}$ & $\begin{array}{l}\text {-Fluid boluses targeted to CVP } \\
\text { 8-12 mmHg. } \\
\text {-Identical algorithm for } \\
\text { noradrenaline, dobutamine and } \\
\text { nitrate use in both groups } \\
\text {-At } 7 \text { days, mean net fluid } \\
\text { balance was } 3974.5 \text { ml } \\
\text {-Estimated mean daily fluid } \\
\text { balance over study period: } 8.1 \\
\text { ml/kg/day }\end{array}$ & $\begin{array}{l}\text {-28 day mortality } \\
\text {-Ventilator-free days } \\
\text {-ICU length of stay } \\
\text {-Maximum SOFA } \\
\text { score } \\
\text {-RRT-free days }\end{array}$ \\
\hline $\begin{array}{l}\text { Richard et al, } \\
2015\end{array}$ & $\mathrm{RCT}$ & $N=60$ & $\begin{array}{l}\text {-Fluid boluses targeted to pulse } \\
\text { pressure variation }<13 \% \text { (if }\end{array}$ & $\begin{array}{l}\text {-Fluid boluses targeted to CVP } \\
\geq 8 \mathrm{mmHg} \text { for duration of shock }\end{array}$ & $\begin{array}{l}-28 \text { day mortality } \\
\text {-Ventilator-free days }\end{array}$ \\
\hline
\end{tabular}




\begin{tabular}{|c|c|c|c|c|c|}
\hline & $\begin{array}{l}\text { Single } \\
\text { centre in } \\
\text { France }\end{array}$ & $\begin{array}{l}\text { Inclusion criteria: } \\
\text {-Age } \geq 18 \text { years } \\
\text {-Septic shock } \\
\text {-Pre-enrollment fluid } \\
\text { loading } \geq 25 \mathrm{ml} / \mathrm{kg} \text { body } \\
\text { weight } \\
\text {-Onset of hypotension } \\
<12 \text { hours pre-enrollment } \\
\text { Exclusion criteria: } \\
\text {-Pregnancy } \\
\text {-Acute coronary } \\
\text { syndrome or cardiogenic } \\
\text { pulmonary oedema } \\
\text {-Acute cerebral event <30 } \\
\text { days } \\
\text {-Cannulation } \\
\text { contraindicated } \\
\text {-Uncontrolled } \\
\text { haemorrhage, need for } \\
\text { immediate surgery } \\
\text {-Trauma or burns }>20 \% \\
\text { BSA } \\
\text {-Previous inclusion in } \\
\text { RCT } \\
\text {-Limitation of treatment } \\
\text {-Absence of consent, } \\
\text { legal protection order or } \\
\text { lack of social security }\end{array}$ & $\begin{array}{l}\text { criteria for PPV use met) and } \Delta \\
\text { stroke volume }<10 \% \text { in } \\
\text { response to passive leg raise } \\
\text { manoeuver for duration of shock } \\
\text {-Identical protocol for use of } \\
\text { noradrenaline, dobutamine, and } \\
\text { red blood cells } \\
\text {-Median daily fluid balance for } \\
\text { duration of shock was } 888 \mathrm{ml} \\
\text { (IQR } 153 \text { to } 2816 \text { ml)* } \\
\text {-Estimated mean daily fluid } \\
\text { balance over study period: } 2.6 \\
\text { ml/kg/day }\end{array}$ & $\begin{array}{l}\text { - Identical protocol for use of } \\
\text { noradrenaline, dobutamine, and } \\
\text { red blood cells } \\
\text {-Median daily fluid balance for } \\
\text { duration of shock was } 1749 \mathrm{ml} \\
\text { (IQR } 146 \text { to } 2788 \text { ml)* } \\
\text {-Estimated mean daily fluid } \\
\text { balance over study period: } 3.2 \\
\text { ml/kg/day }\end{array}$ & $\begin{array}{l}\text {-Length of ICU stay } \\
\text { (survivors) } \\
\text {-Number of days with } \\
\text { SOFA } \geq 6\end{array}$ \\
\hline $\begin{array}{l}\text { Hjortrup et al, } \\
2016\end{array}$ & $\begin{array}{l}\text { RCT } \\
\text { Nine } \\
\text { centres in } \\
\text { Denmark } \\
\text { and Finland }\end{array}$ & $\begin{array}{l}\mathrm{N}=151 \\
\text { Inclusion criteria: } \\
\text {-Age } \geq 18 \text { years } \\
\text {-Treated in ICU } \\
\text {-Sepsis with circulatory } \\
\text { impairment } \\
\text {-Fluid bolus administration } \\
\geq 30 \mathrm{ml} / \mathrm{kg} \text { ideal body } \\
\text { weight }\end{array}$ & $\begin{array}{l}\text {-Noradrenaline used to maintain } \\
\text { mean arterial pressure } \geq 65 \\
\text { mmHg or appropriate target } \\
\text { - } 250 \text { to } 500 \mathrm{ml} \text { crystalloid } \\
\text { boluses could be administered } \\
\text { only if evidence of } \\
\text { hypoperfusion (lactate } \geq 4 \\
\mathrm{mmol} / \mathrm{L}, \text { mean arterial pressure } \\
<50 \mathrm{mmHg} \text {, skin mottling beyond }\end{array}$ & $\begin{array}{l}\text {-Noradrenaline used to maintain } \\
\text { mean arterial pressure } \geq 65 \\
\text { mmHg or appropriate target } \\
\text {-Crystalloid boluses could be } \\
\text { administered provided evidence } \\
\text { of fluid responsiveness present } \\
\text { according to static or dynamic } \\
\text { variables of clinician's choice }\end{array}$ & $\begin{array}{l}\text {-90 day mortality } \\
\text {-Ventilator-free days } \\
\text {-Length of ICU stay } \\
\text {-RRT use } \\
\text {-Worsening AKI }\end{array}$ \\
\hline
\end{tabular}




\begin{tabular}{|c|c|c|c|c|}
\hline & & $\begin{array}{l}\text {-Noradrenaline infusion } \\
\text { used to mainatin blood } \\
\text { pressure } \\
\text { Exclusion criteria: } \\
\text {-Receiving RRT (or } \\
\text { deemed imminent) } \\
\text {-Plasma potassium > } 6 \\
\text { mmol/L within last } 6 \text { hours } \\
\text {-Creatinine level > } 350 \\
\mu \text { mol/L } \\
\text {-FiO2 }>0.8 \text { and positive } \\
\text { end expiratory pressure > } \\
10 \text { cmH }{ }_{2} O \\
\text {-Life-threatening bleeding } \\
\text {-Burns }>10 \% \text { BSA } \\
\text {-Lack of commitment to } \\
\text { full life support } \\
\text {-Consent unobtainable } \\
\text {-Kidney or liver transplant } \\
\text { during same admission } \\
\text {-Previous enrollment in } \\
\text { this trial }\end{array}$ & $\begin{array}{l}\text { edge of kneecap, urine output } \leq \\
0.1 \mathrm{ml} / \mathrm{kg} \text { ideal body weight } \\
\text { within } 2 \text { hours of randomisation) } \\
\text {-At } 5 \text { days, median fluid balance } \\
\text { was } 1752 \mathrm{ml} \text { (IQR } 407 \text { to } 5114 \\
\mathrm{ml} \text { ) } \\
\text {-Estimated mean daily fluid } \\
\text { balance over study period: } 5.4 \\
\mathrm{ml} / \mathrm{kg} / \text { day }\end{array}$ & $\begin{array}{l}\text {-At } 5 \text { days, median fluid balance } \\
\text { was } 2680 \mathrm{ml} \text { (IQR -1153 to } 3758 \\
\mathrm{ml} \text { ) } \\
\text {-Estimated mean daily fluid } \\
\text { balance over study period: } 9.1 \\
\mathrm{ml} / \mathrm{kg} / \text { day }\end{array}$ \\
\hline
\end{tabular}

Table 1. Characteristics of included randomised trials. Unless otherwise specified, standard definitions are used for ALI, ARDS, SIRS, sepsis and septic shock. [70-72]. *Denotes studies in which between-group differences in fluid balance was considered to be clinically-significant. Unless otherwise specified, data are presented as mean +/- standard deviation. RCT: Randomised controlled trial; EVLW: Extravascular lung water; PCWP: Pulmonary capillary wedge pressure; MI: Millilitres; IV: intravenous; SOFA: Sequential organ failure assessment; CVP: Central venous pressure; ALI: Acute lung injury; AECC:

American-European Consensus Conference; CRRT: continuous renal replacement therapy; PiCCO: Pulse Index Continuous Cardiac Output; QLQ-C30:

Quality of life questionnaire core-30; COPD: Chronic obstructive pulmonary disease; ITBVI: Intrathoracic blood volume index; IQR: Interquartile range; PPV: Pulse pressure variation; BSA: Body surface area; $\mathrm{FiO}_{2}$ : Fraction of Inspired Oxygen. 


\begin{tabular}{|c|c|c|c|c|c|c|c|c|c|c|c|c|}
\hline \multicolumn{7}{|c|}{ Quality assessment } & \multicolumn{2}{|c|}{ № of patients } & \multicolumn{2}{|c|}{ Effect } & \multirow[t]{2}{*}{ Quality } & \multirow[t]{2}{*}{ Importance } \\
\hline $\begin{array}{l}\text { № of } \\
\text { studies }\end{array}$ & $\begin{array}{l}\text { Study } \\
\text { design }\end{array}$ & Risk of bias & Inconsistency & Indirectness & Imprecision & $\begin{array}{c}\text { Other } \\
\text { considerations }\end{array}$ & $\begin{array}{l}\text { Conservative or } \\
\text { deresuscitative } \\
\text { fluid strategy }\end{array}$ & $\begin{array}{l}\text { Liberal fluid } \\
\text { strategy or } \\
\text { usual care }\end{array}$ & $\begin{array}{l}\text { Relative } \\
(95 \% \mathrm{Cl})\end{array}$ & $\begin{array}{l}\text { Absolute } \\
(95 \% \mathrm{Cl})\end{array}$ & & \\
\hline \multicolumn{13}{|l|}{ Mortality } \\
\hline 11 & $\begin{array}{l}\text { randomised } \\
\text { trials }\end{array}$ & serious ${ }^{1}$ & not serious & very serious ${ }^{2}$ & serious & none & $337 / 973(34.6 \%)$ & $\begin{array}{l}373 / 977 \\
(38.2 \%)\end{array}$ & $\begin{array}{l}\text { RR } 0.92 \\
(0.82 \text { to } 1.03)\end{array}$ & $\begin{array}{c}31 \text { fewer per } \\
1,000 \\
\text { (from } 11 \text { more to } \\
69 \text { fewer) }\end{array}$ & @ERY & CRITICAL \\
\hline \multicolumn{13}{|c|}{ Ventilator free days } \\
\hline 7 & $\begin{array}{l}\text { randomised } \\
\text { trials }\end{array}$ & not serious & not serious & very serious ${ }^{2}$ & not serious & none & 891 & 893 & - & $\begin{array}{c}\text { MD } 1.82 \text { days } \\
\text { more } \\
\text { (0.53 more to } 3.1 \\
\text { more })\end{array}$ & $\begin{array}{l}\text { LOW } \\
\text { LOW }\end{array}$ & IMPORTANT \\
\hline \multicolumn{13}{|c|}{ Intensive Care Unit (ICU) length of stay } \\
\hline 7 & $\begin{array}{l}\text { randomised } \\
\text { trials }\end{array}$ & serious $^{3}$ & serious ${ }^{4}$ & very serious $^{2}$ & not serious & none & 444 & 448 & - & $\begin{array}{c}\text { MD } 1.88 \text { days } \\
\text { fewer } \\
\text { (0.12 fewer to } \\
3.64 \text { fewer) }\end{array}$ & @eRY & IMPORTANT \\
\hline \multicolumn{13}{|c|}{ Renal Replacement Therapy (RRT) use } \\
\hline 3 & $\begin{array}{l}\text { randomised } \\
\text { trials }\end{array}$ & not serious & not serious & very serious ${ }^{2}$ & serious ${ }^{5}$ & none & $83 / 619(13.4 \%)$ & $\begin{array}{l}100 / 614 \\
(16.3 \%)\end{array}$ & $\begin{array}{c}\text { RR } 0.88 \\
\text { (0.64 to } 1.22)\end{array}$ & $\begin{array}{c}20 \text { fewer per } \\
1,000 \\
\text { (from } 36 \text { more to } \\
59 \text { fewer) }\end{array}$ & $\bigcup_{\mathrm{VERY}}$ & CRITICAL \\
\hline \multicolumn{13}{|c|}{ Post-ICU Cognitive function (assessed with: QLQ-C30 congitive function domain; Scale from: 0 to 100, with higher scores denoting better cognitive function) } \\
\hline 1 & $\begin{array}{l}\text { randomised } \\
\text { trials }\end{array}$ & very serious ${ }^{6}$ & not serious & serious $^{7}$ & serious ${ }^{5}$ & none & 50 & 50 & - & $\begin{array}{c}\text { MD } 10.71 \text { Points } \\
\text { higher } \\
\text { (5.22 higher to } \\
16.2 \text { higher) }\end{array}$ & $\begin{array}{l}\text { O } \\
\text { VERY } \\
\text { LOW }\end{array}$ & CRITICAL \\
\hline
\end{tabular}


Table 2. GRADE Summary of findings table for key outcomes. Cl: Confidence interval; RR: Risk ratio; MD Mean difference. Explanatory notes: 1. Only five studies were at low risk of bias, the remainder were at moderate or high risk of bias. 2. Significant variability in populations, interventions and comparators studied. 3. Only two studies were at low risk of bias, the remainder were at moderate or high risk of bias. 4. Considerable heterogeneity present across studies $\left(I^{2}=75 \%\right)$. 5. Insufficient number of participants to exclude clinically important benefit or harm. 6. Single study, uncertain risk of bias across all domains. 7. Limited available information on intervention strategy 
Appendix 1. Summary of included observational studies.

\begin{tabular}{|c|c|c|c|c|c|}
\hline Author & Methods & $\begin{array}{l}\text { Inclusion and } \\
\text { exclusion criteria }\end{array}$ & $\begin{array}{l}\text { Patient } \\
\text { characteristics }\end{array}$ & Key outcomes & Key findings \\
\hline $\begin{array}{l}\text { Abulebda et al, } \\
2014 \text {. }\end{array}$ & $\begin{array}{l}\text { - Secondary } \\
\text { analysis of } \\
\text { multicentre } \\
\text { prospective } \\
\text { observational } \\
\text { study of genomics } \\
\text { in sepsis } \\
\text { - USA }\end{array}$ & $\begin{array}{l}\text { Inclusion: } \\
\text { - Age } \leq 10 \text { years } \\
\text {-Septic shock } \\
\text { - Enrolment in an } \\
\text { ongoing genomic } \\
\text { study } \\
\text { Exclusion: } \\
\text {-None reported }\end{array}$ & $\begin{array}{l}\mathrm{N}=317 \\
\text { Non-survivors: } \\
\text {-Median age 1.3 } \\
\text { yrs. (IQR 0.2-4.5) } \\
\text {-65\% male } \\
\text {-Median PRISM } \\
\text { score } 28 \text { (IQR 17- } \\
\text { 37) } \\
\text { Survivors: } \\
\text {-Median age } 2.9 \\
\text { (IQR 1.1-6.7) } \\
\text {-59\% male } \\
\text {-Median PRISM } \\
\text { score 12 (IQR 7- } \\
\text { 18) }\end{array}$ & -28 day mortality & $\begin{array}{l}\text { - Non-survivors had a higher } \\
\text { cumulative fluid balance } 7 \text { at day } 7 \\
\text { (median } 19.5 \% \text { of body weight, IQR } \\
10.4 \text { to } 40.1 \text { ) compared to survivors } \\
\text { (median } 6.5 \% \text { body weight, IQR }-1.3 \\
\text { to } 14.6 \text { ), } p=<0.001\end{array}$ \\
\hline
\end{tabular}




\begin{tabular}{|c|c|c|c|c|c|}
\hline 2015. & $\begin{array}{l}\text { cohort study } \\
\text { - USA }\end{array}$ & $\begin{array}{l}\text { - Shock states } \\
\text { (majority sepsis or } \\
\text { SIRS) } \\
\text { - Age } \leq 18 \text { years } \\
\text { Exclusion: } \\
\text { - PICU length of } \\
\text { stay < } 48 \text { hours } \\
\text {-Premature } \\
\text { neonates } \\
\text {-Post-operative } \\
\text { congenital heart } \\
\text { disease }\end{array}$ & $\begin{array}{l}\text {-Median age } 1.1 \\
\text { yrs. (Range } 0 \text { - } \\
17.4 \text { ) } \\
-59 \% \text { male } \\
\text {-Median Paediatric } \\
\text { Index of Mortality } \\
2 \text { score } 5.1 \\
\text { (Range } 0.2-99.3 \text { ) } \\
\text {-Sepsis or septic } \\
\text { shock } 83 \%\end{array}$ & $\begin{array}{l}\text {-Duration of } \\
\text { mechanical } \\
\text { ventilation } \\
\text { (survivors) } \\
\text {-Renal } \\
\text { replacement } \\
\text { therapy use } \\
\text {-Length of ICU } \\
\text { stay }\end{array}$ & $\begin{array}{l}\text { mortality included } \\
\text { presence of fluid overload ( } \geq 10 \% \\
\text { body weight at } 3 \text { days) (adjusted OR } \\
9.17,95 \% \mathrm{Cl} 2.22-55.57 \text { ); peak fluid } \\
\text { overload within } 7 \text { days (adjusted OR } \\
1.13 \text { per } \% \text { body weight, } 95 \% \mathrm{Cl} \\
1.07-1.23 \text { ); and duration of fluid } \\
\text { overload (adjusted OR } 1.61 \text { per day, } \\
95 \% \mathrm{Cl} 1.21-2.28 \text { ) } \\
\text { - Compared with matched controls, } \\
\text { cases with fluid overload } \geq 10 \% \text { body } \\
\text { weight at } 3 \text { days, had higher } \\
\text { mortality ( } 37 \% \text { versus } 3 \%, P=0.002 \text { ); } \\
\text { similar duration of mechanical } \\
\text { ventilation (median } 6 \text { days versus } 5 \\
\text { days, } P=0.36 \text { ), similar rates of RRT } \\
\text { use (37\% vs } 13 \%, P=0.07 \text { ) and } \\
\text { similar length of ICU stay (median } 9 \\
\text { days versus } 8 \text { days, } P=0.73 \text { ). }\end{array}$ \\
\hline
\end{tabular}




\begin{tabular}{|c|c|c|c|c|c|}
\hline & & $\begin{array}{l}\text { haemorrhage, GI } \\
\text { or other fluid loss } \\
\text { - Pregnancy }\end{array}$ & & & \\
\hline $\begin{array}{l}\text { Botdorf et al, } \\
2015 .\end{array}$ & $\begin{array}{l}\text { - Single centre } \\
\text { retrospective } \\
\text { cohort study } \\
\text { - USA }\end{array}$ & $\begin{array}{l}\text { Inclusion: } \\
\text {-Suspected sepsis } \\
\text {-ICU stay > 24hrs } \\
\text { Exclusion: } \\
\text { - None reported }\end{array}$ & $\begin{array}{l}\mathrm{N}=162 \\
\text {-Median age 68 } \\
\text { yrs. (IQR 58-79) } \\
\text {-55\% male } \\
\text {-Median APACHE } \\
\text { 4 score 83 (IQR } \\
67-104 \text { ) }\end{array}$ & -ICU mortality & $\begin{array}{l}\text { - Net fluid balance at } 48 \text { hours was } \\
\text { higher in non-survivors compared to } \\
\text { survivors (median } 8790 \mathrm{ml} \text {, IQR } \\
4530 \text { to } 11400 \text { vs median } 5380 \mathrm{ml} \text {, } \\
\text { IQR } 2900 \text { to } 7820, p=0.023 \text { ) }\end{array}$ \\
\hline Boyd et al, 2011. & $\begin{array}{l}\text {-Secondary } \\
\text { analysis of a } \\
\text { multicentre } \\
\text { randomised } \\
\text { controlled trial of } \\
\text { vasopressin } \\
\text { versus } \\
\text { norepinephrine in } \\
27 \text { centres in } \\
\text { Canada, Australia } \\
\text { and USA }\end{array}$ & $\begin{array}{l}\text { Inclusion: } \\
\text {-Septic shock } \\
\text {-Minimum of } \\
5 \text { mcg/min } \\
\text { noradrenaline } \\
\text { infusion } \\
\text { Exclusion: } \\
\text { - Unstable } \\
\text { coronary } \\
\text { syndrome } \\
\text {->24 hours since } \\
\text { enrolment criteria } \\
\text { met } \\
\text {-Estimated } 6 \\
\text { month mortality } \\
>50 \% \\
\text {-Suspected or } \\
\text { proven mesenteric } \\
\text { ischaemia } \\
\text { - Underlying } \\
\text { chronic heart } \\
\text { disease } \\
\text {-Anticipation of } \\
\text { imminent death or } \\
\text { lack of } \\
\text { commitment to } \\
\text { aggressive care }\end{array}$ & $\begin{array}{l}\mathrm{N}=778 \\
\text { Not reported for } \\
\text { overall cohort }\end{array}$ & - 28-day mortality & $\begin{array}{l}\text { - Higher net fluid balance at } 4 \text { days } \\
\text { (and at } 12 \text { hours) was an } \\
\text { independent risk factor for mortality: } \\
\text { adjusted hazard ratios by quartiles } \\
\text { with decreasing fluid balances } 0.739 \\
(95 \% \mathrm{Cl} 0.503-1.087), 0.512(0.339- \\
0.775), 0.466(0.299-0.724) \text {. }\end{array}$ \\
\hline
\end{tabular}




\begin{tabular}{|c|c|c|c|c|c|}
\hline Chen et al, 2011. & $\begin{array}{l}\text {-Single centre } \\
\text { retrospective } \\
\text { cohort study } \\
\text {-China }\end{array}$ & $\begin{array}{l}\text { Inclusion: } \\
\text {-Septic shock } \\
\text { (ACP/SCCM } \\
\text { criteria) } \\
\text { Exclusion: } \\
\text {-Fluid bolus or } \\
\text { vasopressor } \\
\text { administration in } \\
\text { another hospital }\end{array}$ & $\begin{array}{l}\mathrm{N}=107 \\
\text { Survivors }(\mathrm{n}=68) \text { : } \\
\text {-Age } 66.7 \text { years } \\
+/-14.5 \\
-78 \% \text { male } \\
\text {-APACHE score } \\
\text { 14.7+/-3.1 } \\
\text {-SOFA score } 6.5 \\
+/-1.5 \\
\text { Non-survivors } \\
\text { (n=39) } \\
\text {-Age } 68.88+/- \\
13.1 \text { years } \\
\text {-69\% male } \\
\text {-APACHE score } \\
16.3+/-3.6 \\
\text {-SOFA score } 7.2 \\
+/-1.5\end{array}$ & -28 day mortality & $\begin{array}{l}\text {-Absence of conservative late fluid } \\
\text { management, negative fluid balance } \\
\text { in first week }<2 \text { litres, and total } \\
\text { intake in first week }>20 \text { litres were } \\
\text { independent risk factors for mortality. }\end{array}$ \\
\hline
\end{tabular}




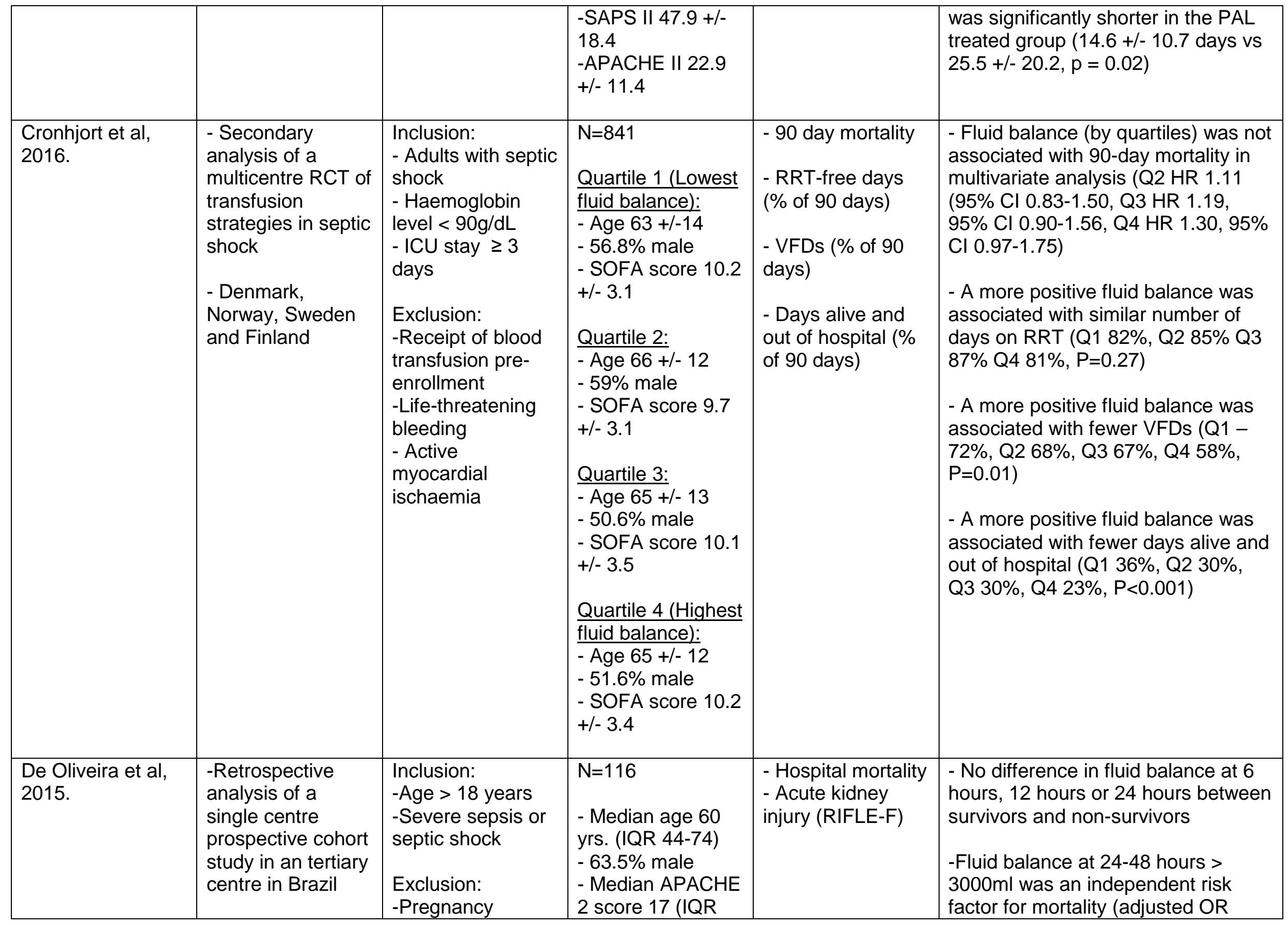




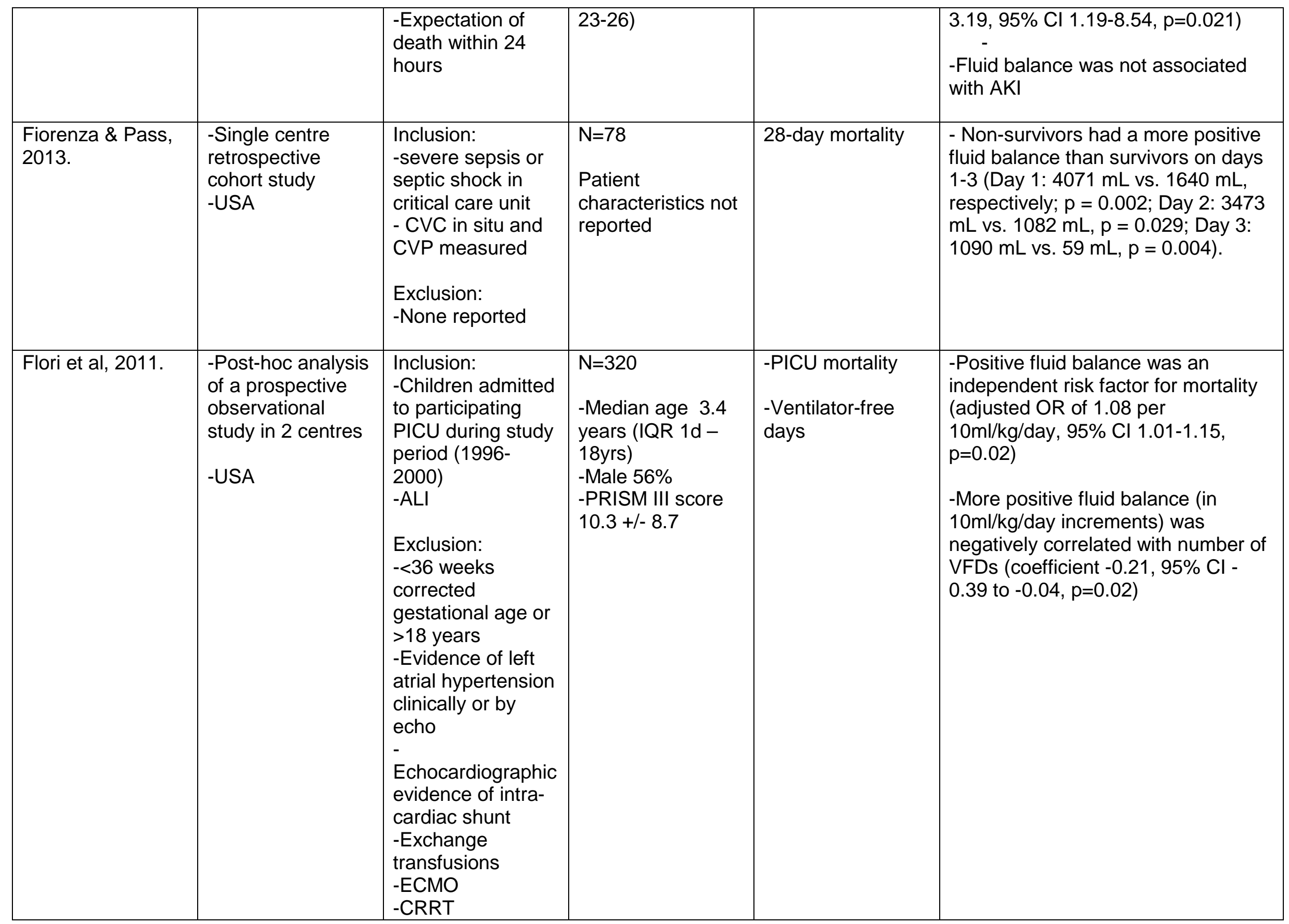




\begin{tabular}{|c|c|c|c|c|c|}
\hline $\begin{array}{l}\text { Grissom et al, } \\
2015 .\end{array}$ & $\begin{array}{l}\text {-Retrospective } \\
\text { analysis of data } \\
\text { from } 4 \text { large } \\
\text { RCTs } \\
\text {-Multicentre data, } \\
\text { mainly from USA } \\
\text { - 3-way } \\
\text { comparison of } \\
\text { outcomes using } \\
\text { comparing a } \\
\text { simplified } \\
\text { conservative fluid } \\
\text { management } \\
\text { protocol (FACTT } \\
\text { Lite) with } \\
\text { conservative and } \\
\text { liberal fluid } \\
\text { regimens from } \\
\text { FACTT trial [15] }\end{array}$ & $\begin{array}{l}\text { Inclusion: } \\
\text {-Enrolment in one } \\
\text { of } 4 \text { randomised } \\
\text { trials in ARDS - } \\
\text { FACTT, EDEN, } \\
\text { OMEGA and } \\
\text { ALTA. } \\
\text { - presence of CVC } \\
\text { Exclusion: } \\
\text {-Chronic dialysis } \\
\text { dependence }\end{array}$ & $\begin{array}{l}\mathrm{N}=2124 \\
\text { FACTT Lite group: } \\
\text {-Age } 51.5 \text { yrs. +/- } \\
0.5 \\
\text {-Male } 52 \% \\
\text {-APACHE } 3 \text { score } \\
91.0+/-0.8 \\
\text { FACTT } \\
\frac{\text { conservative: }}{\text {-Age } 50.1 \text { yrs. +/- }} \\
0.7 \\
\text {-Male } 52 \% \\
\text {-APACHE } 3 \text { score } \\
93.1+/-1.4 \\
\text { FACTT liberal: } \\
\text {-Age } 49.5 \text { yrs. +/- } \\
0.7 \\
-55 \% \text { male } \\
\text {-APACHE } 3 \text { score } \\
95.2+/-1.4\end{array}$ & $\begin{array}{l}\text { - } 60 \text { day mortality } \\
\text { - Ventilator free } \\
\text { days } \\
\text {-ICU-free days } \\
\text {-Acute kidney } \\
\text { injury (increase in } \\
\text { serum creatinine } \\
\text { by } \geq 0.3 \mathrm{mg} / \mathrm{dl} \text { or } \\
\text { by } \geq 50 \% \text { ) }\end{array}$ & $\begin{array}{l}\text {-Fluid balance at } 7 \text { days in the } \\
\text { FACCT lite group was intermediate } \\
\text { between the two arms of the FACCT } \\
\text { trial [15] (1918 +/-323 ml versus }-136 \\
+/-491 \text { ml and } 6992+/-502 \mathrm{ml}) \text {. } \\
\text { - 60-day mortality was similar in all } 3 \\
\text { cohorts (FACTT Lite vs FACTT } \\
\text { Liberal } p=0.56 \text {, FACTT Lite vs } \\
\text { FACTT Conservative p=0.91) after } \\
\text { adjustment for age and severity of } \\
\text { illness } \\
\text {-The number of ventilator free days } \\
\text { was similar between FACCT Lite } \\
\text { and FACTT conservative groups, but } \\
\text { there were more in FACTT Lite } \\
\text { compared to FACTT Liberal (14.9+/- } \\
\text { 0.3 vs } 12.1+/-0.5 \text { days, P<0.001). } \\
\text {-The number of ICU free days was } \\
\text { similar between FACCT Lite and } \\
\text { FACTT Conservative, but there were } \\
\text { more in FACTT Lite compared to } \\
\text { FACTT Liberal (14.4 +/-0.3 vs } 11.2 \\
+/-0.4, \text { P }<0.001 \text { ) } \\
\text { - Acute kidney injury rates (adjusted } \\
\text { for fluid balance) were similar } \\
\text { between FACCT Lite and FACTT } \\
\text { Conservative groups, but were lower } \\
\text { in FACTT Lite compared to FACTT } \\
\text { Liberal ( } 56 \% \text { vs } 66 \% \text {, p=<0.001) }\end{array}$ \\
\hline $\begin{array}{l}\text { Herrera Gutierrez } \\
\text { et al, } 2013 .\end{array}$ & $\begin{array}{l}\text {-Single centre } \\
\text { prospective cohort } \\
\text { study } \\
\text {-Spain }\end{array}$ & $\begin{array}{l}\text { Inclusion: } \\
\text {-Sepsis } \\
\text { (undefined) } \\
\text { Exclusion: } \\
\text {-Unclear }\end{array}$ & $\begin{array}{l}\mathrm{N}=129 \\
\text {-Age } 56.8 \mathrm{yrs}+/- \\
16.7 \\
-58.8 \% \text { male } \\
\text {-APACHE } 2 \text { score }\end{array}$ & $\begin{array}{l}\text {-28 day mortality } \\
\text {-Acute kidney } \\
\text { injury (KDIGO) } \\
\text { during ICU stay }\end{array}$ & $\begin{array}{l}\text {-Increasing severity of AKI was } \\
\text { associated with more positive fluid } \\
\text { balance (as percentage of body } \\
\text { weight) at day 3: } 0.23 \%+/-6.3 \\
\text { (KDIGO Stage } 0 \text { ), } 6.65 \%+/-2.15 \\
\text { (KDIGO Stage 1, } \mathrm{P}=0.08 \text { ), } 6.95 \%+/ \text { - }\end{array}$ \\
\hline
\end{tabular}




\begin{tabular}{|c|c|c|c|c|c|}
\hline & & & $\begin{array}{l}23.9+/-7.7 \text { at } \\
\text { admission } \\
\text {-SOFA score } 12.2 \\
+/-5.1 \text { at } \\
\text { admission }\end{array}$ & & $\begin{array}{l}\text { 1.67 (KDIGO Stage 2, } \mathrm{P}=0.08 \text { ), } \\
7.87 \%+/-6.29 \text { (KDIGO Stage } 3 \text {, } \\
\mathrm{P}<0.001) \\
\text {-Day } 3 \text { net fluid balance (as } \% \text { of } \\
\text { total body weight) was significantly } \\
\text { lower in survivors than non-survivors } \\
(0.7 \%+/-6.4 \text { vs } 6.7 \%+/-6.6 \text {, } \\
\mathrm{P}<0.0001) \\
\text {-Day } 3 \text { net fluid balance was an } \\
\text { independent risk factor for mortality } \\
\text { (adjusted OR } 1.13,95 \% \mathrm{Cl} 1.06- \\
1.22 \text { - }\end{array}$ \\
\hline $\begin{array}{l}\text { Kongsayreepong } \\
\text { \& Nitikaroon, } \\
2013 .\end{array}$ & $\begin{array}{l}\text {-Prospective } \\
\text { single centre } \\
\text { cohort study } \\
\text {-Thailand }\end{array}$ & $\begin{array}{l}\text { Inclusion: } \\
\text { - Post noncardiac } \\
\text { surgery } \\
\text { - Age } \geq 18 \text { years } \\
\text {-Severe sepsis or } \\
\text { septic shock } \\
\text { Exclusion: } \\
\text {-None reported }\end{array}$ & $\begin{array}{l}\mathrm{N}=196 \\
\text { Patient } \\
\text { characteristics not } \\
\text { reported }\end{array}$ & $\begin{array}{l}\text {-Acute kidney } \\
\text { injury (Acute } \\
\text { Kidney Injury } \\
\text { Network score } \geq \\
\text { stage 1) }\end{array}$ & $\begin{array}{l}\text {-Fluid overload was not an } \\
\text { independent risk factor for AKI. }\end{array}$ \\
\hline $\begin{array}{l}\text { Koonrangsesomboo } \\
\text { n \& Khwannimit, } \\
2015 .\end{array}$ & $\begin{array}{l}\text {-Single centre } \\
\text { retrospective } \\
\text { cohort study } \\
\text {-Thailand }\end{array}$ & $\begin{array}{l}\text { Inclusion: } \\
\text { - Septic shock } \\
\text { requring ICU } \\
\text { Exclusion: } \\
\text { - ICU length of } \\
\text { stay < } 24 \text { hours }\end{array}$ & $\begin{array}{l}\mathrm{N}=1048 \\
\text { - Median age 59 } \\
\text { (IQR 44.75 - 73) } \\
\text { - 58.3\% Male } \\
\text { - Median APACHE } \\
\text { II score } 27 \text { (21- } \\
\text { 34.5) } \\
\text {-Median SOFA } \\
\text { score } 10 \text { (IQR 8- } \\
\text { 13) }\end{array}$ & $\begin{array}{l}\text { - ICU mortality } \\
\text { - Hospital mortality }\end{array}$ & $\begin{array}{l}\text { - After grouping based on } 72 \text {-hour } \\
\text { fluid balance quartiles, the } 3^{\text {rd }} \text { and } 4^{\text {th }} \\
\text { quartiles were independently } \\
\text { associated with ICU mortality } \\
\text { (adjusted OR } 3.04 \text { [95\% CI } 1.90- \\
4.84] \text { and } 4.16 \text { [95\% } \mathrm{Cl} 2.49-6.95] \\
\text { per litre respectively. } \\
\text { - After grouping based on } 72 \text {-hour } \\
\text { fluid balance, the } 3^{\text {rd }} \text { and } 4^{\text {th }} \text { quartiles } \\
\text { were independently associated with } \\
\text { hospital mortality (adjusted OR } 2.75 \\
\text { [95\% Cl 1.74-4.36] and } 3.16 \text { [95\% Cl } \\
1.87-5.35] \text { per litre respectively. }\end{array}$ \\
\hline
\end{tabular}




\begin{tabular}{|c|c|c|c|c|c|}
\hline & $\begin{array}{l}\text { retrospective } \\
\text { cohort study } \\
\text { - USA }\end{array}$ & $\begin{array}{l}\text {-Septic shock } \\
\text {-Transthoracic } \\
\text { echocardiogram } \\
\text { performed within } \\
24 \text { hours of onset } \\
\text { of shock } \\
\text { Exclusion: } \\
\text {-Pre-existing non- } \\
\text { sepsis related } \\
\text { cardiovascular } \\
\text { compromise } \\
\text {-ECMO or VAD } \\
\text { use } \\
\text { - Shock onset at } \\
\text { outside hospital } \\
\text { prior to transfer }\end{array}$ & $\begin{array}{l}\text { Survivors: } \\
\text {-Age } 58.5 \text { yrs. +/- } \\
14.6 \\
\text { - Male } 46.3 \% \\
\text {-APACHE } 2 \text { score } \\
21.7+/-6.3 \\
\text { Non-survivors: } \\
\text {-Age } 63.0 \text { yrs. +/- } \\
14.0 \\
\text {-male } 44.8 \% \\
\text {-APACHE } 2 \text { score } \\
25.1+/-6.7\end{array}$ & & $\begin{array}{l}\text { fluid balance in the } 8 \text { days following } \\
\text { shock onset (median } 7742 \mathrm{ml}[2914- \\
\text { 15992] versus } 3286.5 \mathrm{ml}[1508.5- \\
7467], \mathrm{P}<0.001 \text { ) } \\
\text { - The quartile with the highest fluid } \\
\text { balance had significantly higher } \\
\text { mortality risk ( } \mathrm{P}<0.001 \text { compared to } \\
\text { the lowest fluid balance quartile) } \\
\text {-Being in the highest fluid balance } \\
\text { quartile was an independent risk } \\
\text { factor for death (adjusted OR } 1.66 \\
\text { [1.39-1.98], } P=0.004 \text { ) }\end{array}$ \\
\hline Murai et al, 2014. & $\begin{array}{l}\text { - Multicentre } \\
\text { retrospective } \\
\text { cohort study } \\
\text { - Japan }\end{array}$ & $\begin{array}{l}\text { Inclusion: } \\
\text { - ARDS } \\
\text { - Mechanical } \\
\text { ventilation } \\
\text { - Transpulmonary } \\
\text { thermodilution } \\
\text { monitoring used } \\
\text { Exclusion: } \\
\text { - None reported }\end{array}$ & $\begin{array}{l}\mathrm{N}=207 \\
\text { Patient } \\
\text { characteristics not } \\
\text { reported }\end{array}$ & - 28 day mortality & $\begin{array}{l}\text { - Fluid balance after } 3 \text { days was } \\
\text { higher in non-survivors than } \\
\text { survivors, both before }(5.1+/-4.3 \mathrm{~L} \\
\text { vs } 3.5+/-0.4 \mathrm{~L}, \mathrm{P}=0.03 \text { ) and after } \\
\text { exclusion of patients with SOFA-CV } \\
\text { or SOFA-renal score }>2(3.8+/-1.6 \\
\mathrm{L} \text { vs } 2.2+/-4.0 \mathrm{~L}, \mathrm{P}=0.03) \text {. } \\
\text { - Fluid balance after } 3 \text { days was an } \\
\text { independent predictor of } 28 \text { day } \\
\text { mortality (adjusted OR } 1.0001,95 \% \\
\mathrm{Cl} 1.000017-1.00022, \mathrm{P}=0.03)\end{array}$ \\
\hline $\begin{array}{l}\text { Murphy et al, } \\
2009 \text {. }\end{array}$ & $\begin{array}{l}\text {-Retrospective } \\
\text { cohort study } \\
\text { - } 2 \text { academic } \\
\text { centres in USA }\end{array}$ & $\begin{array}{l}\text { Inclusion: } \\
\text {-Septic shock } \\
\text { - ALI (AECC } \\
\text { definition) } \\
\text {-Mechanical } \\
\text { ventilation > } 24 \\
\text { hrs. } \\
\text { Exclusion: } \\
\text {-Hospitalisation for }\end{array}$ & $\begin{array}{l}\mathrm{N}=212 \\
\text { Survivors: } \\
\text { - Age } 58.5 \mathrm{yrs}+/- \\
15.8 \\
\text { - } 62 \% \text { male } \\
\text { - APACHE } 2 \text { score } \\
23.9+/-6.0 \\
\text { - SOFA score } 9.5 \\
+/-2.5\end{array}$ & - Hospital mortality & $\begin{array}{l}\text {-Net fluid balance after } 7 \text { days was } \\
\text { higher in non-survivors than } \\
\text { survivors (median 13,694 ml; IQR } \\
7113-20249 \text { vs } 8062 \mathrm{ml} \text {; IQR 2412- } \\
\text { 13833, p < 0.001). } \\
\text {-Absence of 'conservative late fluid } \\
\text { management' (defined as even to } \\
\text { negative fluid balance on } \geq 2 \\
\text { consecutive days) was an }\end{array}$ \\
\hline
\end{tabular}




\begin{tabular}{|c|c|c|c|c|c|}
\hline & & $\begin{array}{l}>7 \text { days following } \\
\text { septic shock onset } \\
\text { - Reason for } \\
\text { cardiovascular } \\
\text { compromise other } \\
\text { than septic shock } \\
\text { - ECMO or VAD } \\
\text { use } \\
\text { - Onset of septic } \\
\text { shock while at } \\
\text { non-participating } \\
\text { hospital }\end{array}$ & $\begin{array}{l}\text { Non-survivors: } \\
\text {-Age } 60.7 \text { yrs }+/- \\
14.9 \\
-47 \% \text { male } \\
\text {-APACHE } 2 \text { score } \\
26.7+/-7.3 \\
\text { - SOFA score } 11.0 \\
+/-3.3\end{array}$ & & $\begin{array}{l}\text { independent risk factor for hospital } \\
\text { mortality (adjusted OR } 6.13,95 \% \mathrm{Cl} \\
2.77-13.57, \mathrm{P}<0.001 \text { ) } \\
\text {-Absence of 'adequate initial fluid } \\
\text { resuscitation' (defined as } \geq 20 \mathrm{ml} / \mathrm{kg} \\
\text { fluid bolus before administration of } \\
\text { vasopressors and CVP } \geq 8 \mathrm{mmHg} \\
\text { within } 6 \text { hours of shock onset) was } \\
\text { also an independent risk factor for } \\
\text { mortality (adjusted OR } 4.94,95 \% \mathrm{Cl} \\
2.07-11.79, \mathrm{P}<0.001 \text { ) }\end{array}$ \\
\hline $\begin{array}{l}\text { Perez-Fernandez } \\
\text { et al, } 2011 .\end{array}$ & $\begin{array}{l}\text {-Prospective } \\
\text { single centre } \\
\text { cohort study } \\
\text {-Spain }\end{array}$ & $\begin{array}{l}\text { Inclusion: } \\
\text {-Septic shock } \\
\text {-Acute renal failure } \\
\text { on CRRT for }>24 \\
\text { hours } \\
\text { Exclusion: } \\
\text { - None reported }\end{array}$ & $\begin{array}{l}\mathrm{N}=262 \\
\text {-Age } 62 \mathrm{yrs}+/-13 \\
-69.8 \% \text { male } \\
\text {-APACHE } 2 \text { score } \\
26+/-8 \\
\text {-SOFA score } 12 \\
+/-3.8 \\
-57 \% \text { medical, } \\
43 \% \text { surgical } \\
-87.9 \% \\
\text { mechanically } \\
\text { ventilated }\end{array}$ & -90-day mortality & $\begin{array}{l}\text {-Mortality higher in positive balance } \\
\text { group ( }>+ \text { ve } 1000 \mathrm{ml} / \text { initial } 24 \mathrm{hr} \text { on } \\
\text { initial } 24 \text { hours of CRRT) compared } \\
\text { with "isovolaemic" group }(<+ \text { ve } \\
1000 \mathrm{ml} / \text { initial } 24 \mathrm{hr} \text { on CRRT) }(70.8 \% \\
\text { vs } 55 \%) \text {. } \\
\text {-Positive fluid balance over } 24 \mathrm{hrs} \text { on } \\
\text { CRRT was an independent risk } \\
\text { factor for } 90 \text { day mortality ( }<< \\
0.0001)\end{array}$ \\
\hline $\begin{array}{l}\text { Raimundo et al, } \\
2012 .\end{array}$ & $\begin{array}{l}\text {-Single centre } \\
\text { retrospective } \\
\text { cohort study } \\
\text {-Portugal }\end{array}$ & $\begin{array}{l}\text { Inclusion: } \\
\text {-All patients } \\
\text { admitted to ICU } \\
\text { over } 1 \text { year due to } \\
\text { sepsis } \\
\text { Exclusion: } \\
\text { - ICU stay <24 } \\
\text { hours }\end{array}$ & $\begin{array}{l}\mathrm{N}=68 \\
- \text { Age } 63.4 \text { yrs. }+/- \\
16.2 \\
-73.5 \% \text { male } \\
\text {-APACHE } 2 \text { score } \\
20.1+/-10.3 \\
\text {-SOFA score } 7.1 \\
+/-3.4\end{array}$ & $\begin{array}{l}\text {-ICU mortality } \\
\text {-Incidence of } \\
\text { ARDS } \\
\text {-ICU Length of } \\
\text { stay } \\
\text {-Duration of } \\
\text { mechanical } \\
\text { ventilation }\end{array}$ & $\begin{array}{l}\text {-Comparison between liberal } \\
\text { (positive fluid balance at ICU } \\
\text { discharge, } n=47 \text { ) and conservative } \\
\text { fluid management (neutral or } \\
\text { negative fluid balance at ICU } \\
\text { discharge, } n=21 \text { ) } \\
\text {-ICU mortality } 39.7 \% \text { with higher } \\
\text { mortality in liberal vs conservative } \\
\text { fluid balance group (55.3\% vs } 4.8 \% \text {, } \\
\text { P not reported) } \\
\text {-ARDS more common in the liberal }\end{array}$ \\
\hline
\end{tabular}




\begin{tabular}{|c|c|c|c|c|c|}
\hline & & & & & $\begin{array}{l}\text { vs conservative fluid balance group } \\
\text { ( } 25.5 \% \text { vs } 14.3 \%, P \text { not reported) } \\
\text {-No significant difference in ICU } \\
\text { length of stay between groups ( } 10.7 \\
+/-8.8 \text { vs } 16.5+/-4.9 \text { days, } \mathrm{P} \text { not } \\
\text { reported) } \\
\text {-No significant difference in duration } \\
\text { of mechanical ventilation between } \\
\text { groups }(9.2+/-8.1 \text { vs } 10.2+/-8.2 \\
\text { days, } P \text { not reported) }\end{array}$ \\
\hline $\begin{array}{l}\text { Rodriguez et al, } \\
2013 \text {. }\end{array}$ & $\begin{array}{l}\text {-Retrospective } \\
\text { single centre } \\
\text { cohort study } \\
\text {-Spain }\end{array}$ & $\begin{array}{l}\text { Inclusion: } \\
\text {-Severe sepsis or } \\
\text { septic shock } \\
\text { Exclusion: } \\
\text {-None reported }\end{array}$ & $\begin{array}{l}\mathrm{N}=99 \\
- \text { Age } 66.68 \text { yrs. }+/- \\
14 \\
- \text { APACHE } 2 \text { score } \\
18.52+/-7 \\
-58.6 \% \text { male }\end{array}$ & $\begin{array}{l}\text {-Mortality (time } \\
\text { point undefined) } \\
\text {-Duration of } \\
\text { mechanical } \\
\text { ventilation } \\
\text {-ICU Length of } \\
\text { stay }\end{array}$ & $\begin{array}{l}\text {-No significant difference in mortality } \\
\text { between group with positive fluid } \\
\text { balance group after } 2 \text { days and } \\
\text { negative fluid balance after } 2 \text { days } \\
\text { group ( } 35.3 \text { vs } 18.6 \%, p=0.072 \text { ) } \\
\text {-Longer duration of mechanical } \\
\text { ventilation in positive fluid balance } \\
\text { group ( } 9.63 \pm 3.10 \text { vs } 5.59 \pm 9.56, p \\
<0.05 \text { ) } \\
\text {-No significant difference in length of } \\
\text { ICU stay between groups }(16.96 \pm \\
15.38 \text { vs } 11.88 \pm 12.72, p=0.085 \text { ). }\end{array}$ \\
\hline $\begin{array}{l}\text { Rosenberg et al, } \\
2008 \text {. }\end{array}$ & $\begin{array}{l}\text {-Secondary } \\
\text { analysis of a } \\
\text { clinical trial } \\
\text { comparing lung } \\
\text { protective } \\
\text { ventilation to } \\
\text { traditional } \\
\text { ventilation in } \\
\text { patients with } \\
\text { ARDS in } 24 \text { US } \\
\text { academic } \\
\text { hospitals [59] }\end{array}$ & $\begin{array}{l}\text { Inclusion: } \\
\text {-ARDS or ALI } \\
\text { (AECC definition) } \\
\text {-Intubated and } \\
\text { receiving } \\
\text { mechanical } \\
\text { ventilation in a } \\
\text { participating } \\
\text { centre } \\
\text {-Fluid balance } \\
\text { data available }\end{array}$ & $\begin{array}{l}\mathrm{N}=794 \\
\text { Survivors: } \\
\text { - Age 48yrs }+/-17 \\
\text { - } 59 \% \text { male } \\
\text {-Acute physiology } \\
\text { score (APS) } 70+/- \\
26 \\
\text { Non-survivors: } \\
\text { - Age 59yrs }+/-16 \\
\text { - } 61 \% \text { male } \\
\end{array}$ & - Hospital mortality & $\begin{array}{l}\text { - Cumulative fluid balance at day } 4 \\
\text { was an independent risk factor for } \\
\text { hospital mortality (adjusted OR } \\
1.034 \text { per litre, } 95 \% \mathrm{Cl} 1.187-1.432 \text {, } \\
\mathrm{P}=0.001 \text { ). } \\
\text { - A negative fluid balance at day } 4 \\
\text { was associated with a lower risk of } \\
\text { hospital mortality (adjusted OR } \\
0.502 \text { per litre, } 95 \% \mathrm{Cl} 0.284-0.887 \\
\mathrm{P}<0.001 \text { ) }\end{array}$ \\
\hline
\end{tabular}




\begin{tabular}{|c|c|c|c|c|c|}
\hline & & $\begin{array}{l}\text { Exclusion: } \\
\text { - >36 hours since } \\
\text { inclusion criteria } \\
\text { met } \\
\text { - Pregnancy } \\
\text { - <18 years } \\
\text { - Participation in } \\
\text { another clinical } \\
\text { trial in }<30 \text { days } \\
\text { - Physician refusal }\end{array}$ & - APS $82+/-26$ & & \\
\hline Saito et al, 2012. & $\begin{array}{l}\text {-Retrospective } \\
\text { cohort study } \\
\text { evaluating } \\
\text { outcomes before } \\
\text { and after } \\
\text { implementation of } \\
\text { an 'optimal fluid } \\
\text { management' } \\
\text { strategy utilising } \\
\text { PiCCO to guide } \\
\text { fluid and diuretic } \\
\text { use } \\
\text {-Single centre } \\
\text {-Japan }\end{array}$ & $\begin{array}{l}\text { Inclusion: } \\
\text {-Severe sepsis or } \\
\text { septic shock } \\
\text { requiring } \\
\text { mechanical } \\
\text { ventilation } \\
\text { Exclusion: } \\
\text { - None reported }\end{array}$ & $\begin{array}{l}\mathrm{N}=96 \\
\text { - Median age } 69.5 \\
\text { (IQR 55.5 - 78.5) } \\
\text { - Median APACHE } \\
\text { 2 score 23.0 (IQR } \\
\text { 19-27) } \\
\text {-Median SOFA } \\
\text { score } 10.0 \text { (7.0- } \\
\text { 12.0) } \\
\text {-75\% septic shock }\end{array}$ & $\begin{array}{l}\text { - Incidence of } \\
\text { ARDS } \\
\text { - Incidence of AKI } \\
\text { (RIFLE-F) } \\
\text { - } 28 \text { day mortality }\end{array}$ & $\begin{array}{l}\text {-OFM group ( } \mathrm{n}=47 \text { ) achieved a } \\
\text { negative fluid balance earlier than } \\
\text { the 'before' group ( } \mathrm{n}=49) \\
\text { - Mortality was similar between } \\
\text { groups ( } 14.3 \% \text { vs } 17.0 \%) \\
\text { - Incidence of ARDS was lower in } \\
\text { the 'OFM' group ( } 20.4 \% \text { vs } 57.4 \% \text {, } \\
\mathrm{P}=0.02 \text { ) } \\
\text { - Incidence of AKI similar between } \\
\text { groups } \\
\text { - OFM was an independent } \\
\text { protective factor for ARDS (adjusted } \\
\text { OR } 0.17, \mathrm{Cl} 0.06-0.51, P=0.001)\end{array}$ \\
\hline $\begin{array}{l}\text { Simmons et al, } \\
1987 .\end{array}$ & $\begin{array}{l}\text { - Prospective } \\
\text { cohort study } \\
\text { - Single centre } \\
\text { - USA }\end{array}$ & $\begin{array}{l}\text { Inclusion: } \\
\text {-ARDS defined as: } \\
\text {-Acute respiratory } \\
\text { failure with } \\
\text { bilateral infiltrates } \\
\text { requiring } \\
\text { intubation and } \\
\text { mechanical } \\
\text { ventilation } \\
\text {-PaO2/FiO2 ratio } \\
<150 \mathrm{mmHg} \text { within } \\
72 \text { hours }\end{array}$ & $\begin{array}{l}\mathrm{N}=113 \\
- \text {-Age } 54.9 \mathrm{yrs}+/- \\
16.7 \\
-67.3 \% \text { male } \\
-54.9 \% \text { medical } \\
\text { ICU }\end{array}$ & - Hospital mortality & $\begin{array}{l}\text { - Overall mortality } 77.9 \% \\
\text { - Survivors had a significantly less } \\
\text { positive fluid balance and } \\
\text { significantly greater weight gain over } \\
14 \text { days in univariate analysis } \\
\text { - By day } 14, \text { survivors were on } \\
\text { average } 9.72 \text { litres less positive than } \\
\text { non-survivors }\end{array}$ \\
\hline
\end{tabular}




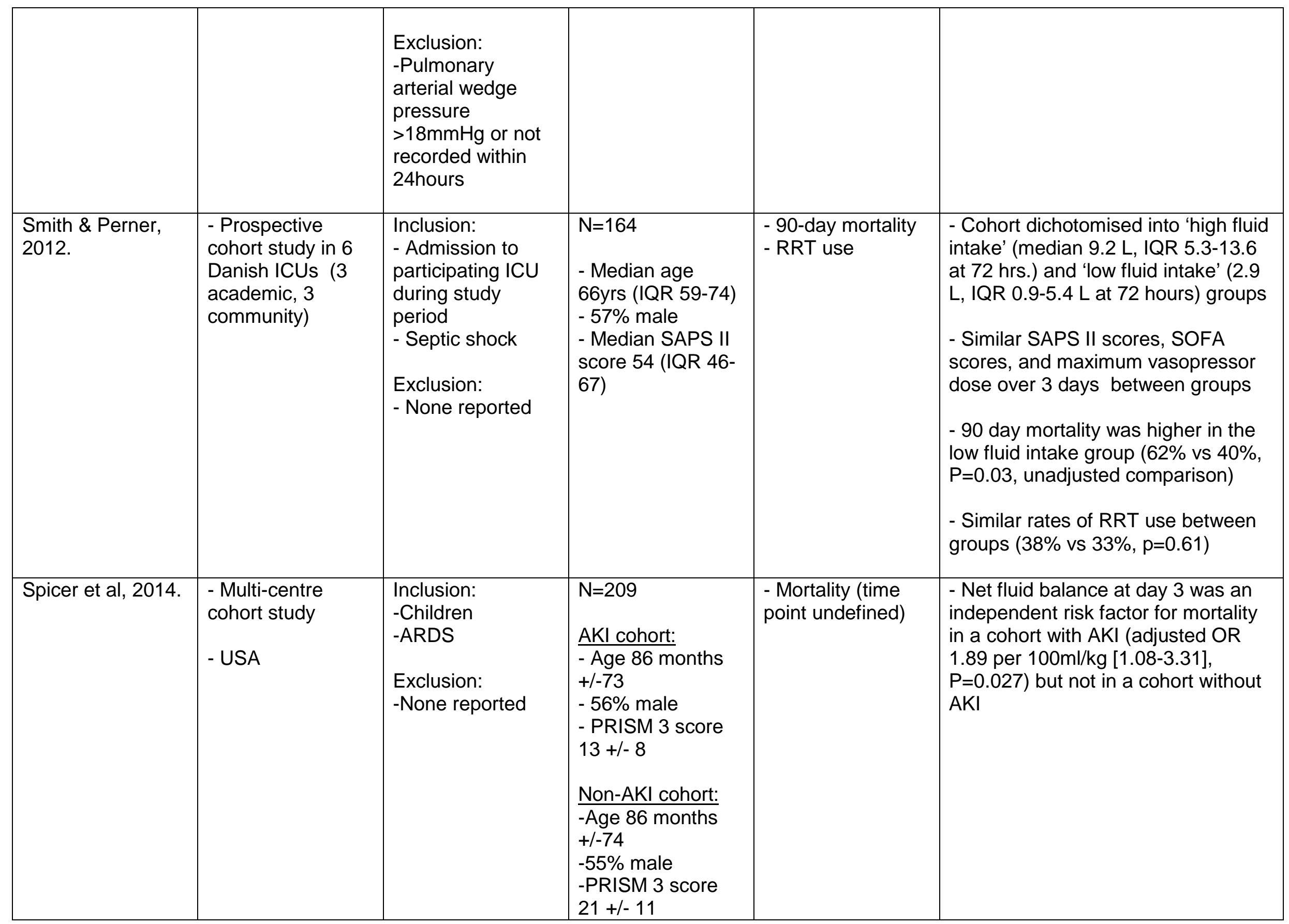




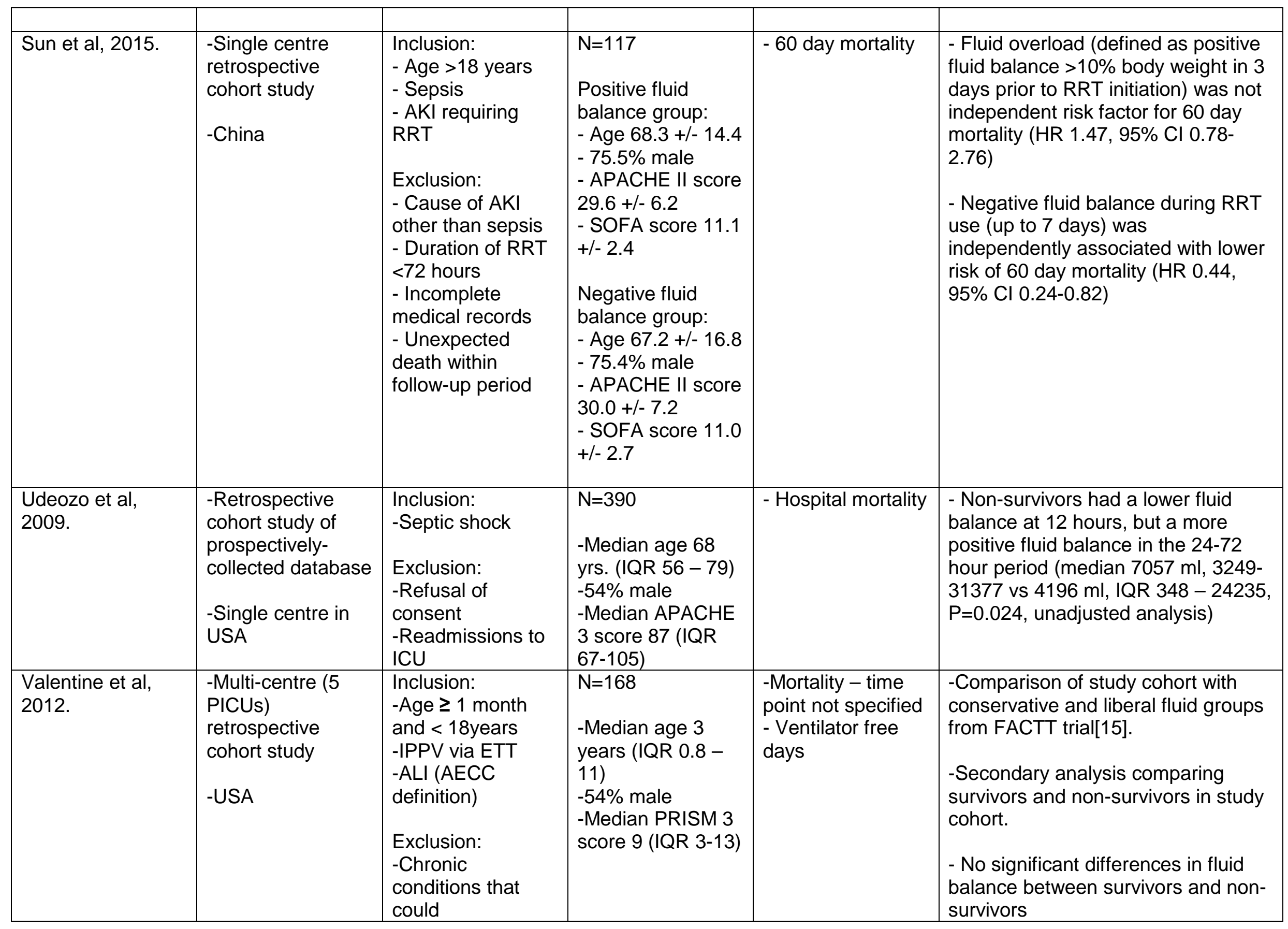




\begin{tabular}{|c|c|c|c|c|c|}
\hline & & $\begin{array}{l}\text { independently } \\
\text { impair weaning, } \\
\text { especially lung or } \\
\text { neuromuscular } \\
\text { conditions } \\
\text {-Cyanotic heart } \\
\text { disease } \\
\text {-Post-lung, renal } \\
\text { or bone marrow } \\
\text { transplant } \\
\text {-Chronic renal } \\
\text { failure } \\
\text {-Burns > 40\% BSA } \\
\text {-Continuous RRT } \\
\text { or ECMO }\end{array}$ & & & $\begin{array}{l}\text { - A more positive fluid balance at day } \\
3 \text { was correlated with fewer VFDs } \\
\text { (coefficient }-0.02, p=0.01 \text { per } \mathrm{ml} / \mathrm{kg} \text { ) } \\
\text { - Total furosemide dose by day } 3 \\
\text { was not associated with number of } \\
\text { VFDs }\end{array}$ \\
\hline $\begin{array}{l}\text { Vincent et al, } \\
\text { 2006. (sepsis) } \\
\text { Sakr et al, } 2005 \\
\text { (ARDS). }\end{array}$ & $\begin{array}{l}\text {-Prospective } \\
\text { multicentre cohort } \\
\text { study } \\
\text {-198 European } \\
\text { ICUs }\end{array}$ & $\begin{array}{l}\text { Inclusion: } \\
\text {-All patients >15 } \\
\text { years admitted to } \\
\text { participating ICU } \\
\text {-Sepsis cohort: } \\
\text { presence of } \\
\text { infection + SIRS } \\
\text { criteria } \\
\text { (ACP/SCCM) } \\
\text {-ALI/ARDS cohort: } \\
\text { AECC criteria } \\
\text { Exclusion: } \\
\text {-Re-admission to } \\
\text { ICU } \\
\text {-Routine post- } \\
\text { operative } \\
\text { admission <24 } \\
\text { hours }\end{array}$ & $\begin{array}{l}\text { Sepsis cohort } \\
\text { (N=1177): } \\
\text {-Median age 65yrs } \\
\text { (IQR 51-74) } \\
\text {-63\% male } \\
\text {-SAPS } 2 \text { score } \\
\text { 42.3 +/- } 16.6 \\
\text {-SOFA score } 6.5 \\
\text { +/- } 4.0 \\
\text { ALI/ARDS cohort } \\
\text { (N=393): } \\
\text {-Age 59 +/- } 17 \\
\text {-60.5\% male } \\
\text {-SAPS } 2 \text { score } \\
\text { 46.6 +/- } 17.6 \\
\text {-Sepsis } 47.5 \%\end{array}$ & -ICU mortality & $\begin{array}{l}\text { Sepsis cohort: } \\
\text {-Net fluid balance at } 72 \text { hours was } \\
\text { an independent risk factor for ICU } \\
\text { mortality (adjusted OR } 1.1 \text { per litre, } \\
95 \% \mathrm{CI} 1.0-1.1, \mathrm{P}<0.001 \text { ) } \\
\text { ALI/ARDS cohort: } \\
\text {-Fluid balance at } 72 \text { hours was } \\
\text { higher for non-survivors than } \\
\text { survivors ( } 3.6+/-6.4 \text { litres vs } 1.8+/- \\
5.4 \text { litres, } p=0.002 \text { ) } \\
\text {-Mean daily fluid balance was an } \\
\text { independent risk factor for ICU } \\
\text { mortality (adjusted OR 1.5 per litre, } \\
95 \% \mathrm{Cl} 1.1-1.9, p=0.003 \text { ) }\end{array}$ \\
\hline Wang et al, 2016. & $\begin{array}{l}\text {-Single centre } \\
\text { retrospective } \\
\text { cohort study } \\
\text {-China }\end{array}$ & $\begin{array}{l}\text { Inclusion: } \\
\text { - Septic shock } \\
\text { - Age } \geq 18 \text { years } \\
\text { - Use of PiCCO } \\
\text { monitoring } \\
\text { - CVP target of 8- }\end{array}$ & $\begin{array}{l}\mathrm{N}=105 \\
\text { Survivors: } \\
\text { - Age } 66+/-17 \\
\text { - } 64.6 \% \text { male } \\
\text { - APACHE II score }\end{array}$ & - 28 day mortality & $\begin{array}{l}\text { - Fluid balance in the } 24 \text { hour and } \\
24-48 \text { hour periods post-initial } \\
\text { resuscitiation both independently } \\
\text { predicted } 28 \text {-day mortality (adjusted } \\
\text { OR } 1.001 \text { per ml, } 95 \% \mathrm{Cl} 1.000- \\
1.001, P=0.016 \text { for } 24 \text { hours; }\end{array}$ \\
\hline
\end{tabular}




\begin{tabular}{|c|c|c|c|c|c|}
\hline & & $\begin{array}{l}12 \mathrm{mmHg} \text { reached } \\
\text { within } 6 \text { hours } \\
\text { - Requirement for } \\
\text { Norepinephrine } \geq \\
0.1 \mathrm{mcg} / \mathrm{kg} / \mathrm{min} \text { or } \\
\text { Dopamine } \geq 5 \\
\mathrm{mcg} / \mathrm{kg} / \mathrm{min} \\
\text { - Survival time } \geq \\
72 \text { hours following } \\
\text { shock onset } \\
\text { Exclusion: } \\
\text {-Pregnancy or } \\
\text { breast-feeding } \\
\text { - Shock in the } \\
\text { absence of } \\
\text { infection } \\
\text { - PiCcO used for } \\
<48 \text { hours or } \\
\text { absent data at } \geq 2 \\
\text { timepoints } \\
\text { - Acute blood loss, } \\
\text { acute myocardial } \\
\text { infarction, } \\
\text { pulmonary } \\
\text { embolism } \\
\text { - Treatment } \\
\text { witheld or } \\
\text { withdrawn during } \\
\text { hopsital stay }\end{array}$ & $\begin{array}{l}21.8+/-7.8 \\
\text { Non-survivors: } \\
\text { - Age } 65+/-18 \\
\text { - } 63.3 \% \text { male } \\
\text { - APACHE II score } \\
25.5+/-6.9\end{array}$ & & $\begin{array}{l}\text { adjusted OR } 1.001 \text { per } \mathrm{ml}, 95 \% \mathrm{Cl} \\
1.000-1.002, \mathrm{P}=0.08 \text { for } 24-48 \\
\text { hours). }\end{array}$ \\
\hline $\begin{array}{l}\text { Wilkowski et al, } \\
1988 .\end{array}$ & $\begin{array}{l}\text {-Single centre } \\
\text { retrospective } \\
\text { cohort study } \\
\text {-Germany }\end{array}$ & $\begin{array}{l}\text { Inclusion: } \\
\text {-ICU patients with } \\
\text { ARDS, defined as } \\
\text { acute respiratory } \\
\text { failure after a } \\
\text { typical insult, } \\
\mathrm{PaO} 2<50 \mathrm{mmHg} \\
\text { with FiO } 2 \geq 0.6 \\
\text { and radiological }\end{array}$ & $\begin{array}{l}\mathrm{N}=124 \\
\text {-Age } 45.4 \text { yrs. } \\
\text { (range 16-78) } \\
-61.3 \% \text { male }\end{array}$ & -ICU mortality & $\begin{array}{l}\text {-No significant difference in mortality } \\
\text { between } 3 \text { treatment groups: (1) } \\
\text { patients treated with diuretics ( } 2 \text { ) } \\
\text { patients treated with haemofiltration } \\
\text { as diuretic-unresponsive, and (3) } \\
\text { patients who died or had } \\
\text { spontaneous diuresis before any } \\
\text { diuretics given }\end{array}$ \\
\hline
\end{tabular}




\begin{tabular}{|c|c|c|c|c|c|}
\hline & & $\begin{array}{l}\text { evidence of } \\
\text { pulmonary } \\
\text { oedema } \\
\text { Exclusion: } \\
\text {-Cardiogenic } \\
\text { pulmonary } \\
\text { oedema } \\
\text {-COPD } \\
\text {-PE } \\
\text {-Pneumonia } \\
\text { without pulmonary } \\
\text { oedema }\end{array}$ & & & $\begin{array}{l}\text {-ICU mortality was higher in patients } \\
\text { with a positive net fluid balance over } \\
\text { the ICU stay }(85.2 \% \text { vs } 66.7 \% \text {, } \\
P<0.05)\end{array}$ \\
\hline $\begin{array}{l}\text { Willson et al, } \\
2015 \text {. }\end{array}$ & $\begin{array}{l}\text {-Secondary } \\
\text { analysis of a large } \\
\text { multi-centre } \\
\text { randomised } \\
\text { controlled trial } \\
\text { investigating } \\
\text { surfactant vs } \\
\text { placebo in } \\
\text { paediatric ARDS } \\
\text {-27 sites in } 6 \\
\text { countries }\end{array}$ & $\begin{array}{l}\text { Inclusion: } \\
\text {-Age <18 years } \\
\text {-ALI of direct } \\
\text { aetiology } \\
\text {-Enrolment in } \\
\text { CARDS trial } \\
\text { (surfactant vs } \\
\text { placebo) within } 48 \\
\text { hours of intubation } \\
\text { Exclusion: } \\
\text {-Indirect lung } \\
\text { injury } \\
\text {-Pre-existing lung } \\
\text { disease } \\
\text {-Limitations on } \\
\text { level of support } \\
\text {-Significant non- } \\
\text { pulmonary organ } \\
\text { dysfunction }\end{array}$ & $\begin{array}{l}\mathrm{N}=109 \\
\text {-Age } 6.1 \text { years +/- } \\
5.8 \\
-51 \% \text { male } \\
\text {-PRISM } 3 \text { score } \\
11.4(+/-6.8)\end{array}$ & -Hospital mortality & $\begin{array}{l}\text {-Cumulative fluid balance at } 7 \text { days } \\
\text { was greater in non-survivors than } \\
\text { survivors }\left(11745 \mathrm{ml} / \mathrm{m}^{2}[10817]\right. \\
\text { versus } 1234 \mathrm{ml} / \mathrm{m}^{2} \text { [2393], P<0.001) }\end{array}$ \\
\hline Yao et al, 2014. & $\begin{array}{l}\text {-Single centre } \\
\text { retrospective } \\
\text { cohort study } \\
\text {-China }\end{array}$ & $\begin{array}{l}\text { Inclusion: } \\
\text {-Septic shock } \\
\text { (ACCP/SCCM } \\
\text { definition) }\end{array}$ & $\begin{array}{l}\mathrm{N}=105 \\
\frac{\text { Survivors }(\mathrm{n}=77)}{\text {-Median age } 64} \\
\text { yrs. (46-74) }\end{array}$ & $\begin{array}{l}\text {-Mortality (time } \\
\text { point undefined) }\end{array}$ & $\begin{array}{l}\text {-Net fluid balance at day } 7 \text { which } \\
\text { was positive or less negative than - } \\
1330 \mathrm{ml} \text { was an independent risk } \\
\text { factor for mortality (adjusted OR } \\
2.98, P=0.037 \text { ) }\end{array}$ \\
\hline
\end{tabular}




\begin{tabular}{|c|c|c|c|c|c|}
\hline & & $\begin{array}{l}\text { Exclusion: } \\
\text { - Age<18 years } \\
\text { - Pregnancy or } \\
\text { lactation }\end{array}$ & $\begin{array}{l}\text {-59.7\% male } \\
\text {-Median APACHE } \\
2 \text { score 15 (14-18) } \\
\text {-Median SOFA } \\
\text { score } 7 \text { (6-8) } \\
\\
\text { Non-survivors } \\
\text { (n=28): } \\
\text {-Median age } 62 \\
\text { yrs. (47-74) } \\
-53.6 \% \text { male } \\
\text {-Median APACHE } \\
2 \text { score 19 (14-21) } \\
\text {-Median SOFA } \\
\text { score } 9 \text { (7-11) }\end{array}$ & & \\
\hline Zhang et al, 2012. & $\begin{array}{l}\text {-Prospective } \\
\text { single-centre } \\
\text { cohort study } \\
\text { investigating the } \\
\text { prognostic utility of } \\
\text { BNP on clinically } \\
\text { important } \\
\text { outcomes and } \\
\text { assessing } \\
\text { correlation } \\
\text { between fluid } \\
\text { balance and } \\
\text { changes in BNP } \\
\text {-China }\end{array}$ & $\begin{array}{l}\text { Inclusion: } \\
\text {-Sepsis } \\
\text {-Age 18-80 years } \\
\text { - PiCCO system in } \\
\text { use for } \\
\text { haemodynamic } \\
\text { monitoring } \\
\text { Exclusion: } \\
\text {-Acute kidney } \\
\text { injury (AKIN Stage } \\
2 \text { or above) } \\
\text {-Patients } \\
\text { considered } \\
\text { moribund or with } \\
\text { DNAR order } \\
\text {-Pre-existing renal } \\
\text { dysfunction }\end{array}$ & $\begin{array}{l}\mathrm{N}=67 \\
\text {-Age 59yrs +/- } 16 \\
-64.2 \% \text { male } \\
\text {-Median APACHE } \\
2 \text { score } 23 \text { (IQR } \\
\text { 19-31) } \\
-46.3 \% \\
\text { mechanically } \\
\text { ventilated }\end{array}$ & -Hospital mortality & $\begin{array}{l}\text {-Net fluid balance at day } 2 \text { was an } \\
\text { independent risk factor for hospital } \\
\text { mortality (OR } 1.50 \text { per } 100 \mathrm{ml}, 95 \% \\
\mathrm{Cl} 1.10-2.04, p=0.01) \text {. } \\
\text {-Change in BNP was correlated with } \\
\text { change in fluid balance (Spearman's } \\
\text { rho }=0.63, p<0.01 \text { ) }\end{array}$ \\
\hline Zhang et al, 2013. & $\begin{array}{l}\text {-Single centre } \\
\text { retrospective } \\
\text { cohort study } \\
\text {-China }\end{array}$ & $\begin{array}{l}\text { Inclusion: } \\
\text {-Sepsis induced } \\
\text { AKI admitted to } \\
\text { ICU } \\
\text { Exclusion: } \\
\text {-Immune }\end{array}$ & $\begin{array}{l}\mathrm{N}=160 \\
\frac{\text { Negative fluid }}{\text { balance group: }} \\
\text {-Age } 51.1 \text { yrs. }+/- \\
18.4 \\
-75.3 \% \text { male }\end{array}$ & $\begin{array}{l}\text {-28 day mortality } \\
\text {-Length of ICU } \\
\text { stay }\end{array}$ & $\begin{array}{l}\text {-Positive fluid balance (defined as no } \\
\text { days of negative balance }>500 \mathrm{ml} \text { in } \\
\text { first } 3 \text { days of ICU stay) was } \\
\text { associated with higher mortality } \\
(68.4 \% \text { vs } 37 \%, p<0.01) \text {, and a } \\
\text { longer ICU stay }(10.1+/-4.9 \text { days vs } \\
12.4+/-8.0 \text { days, } p<0.05 \text { ) }\end{array}$ \\
\hline
\end{tabular}




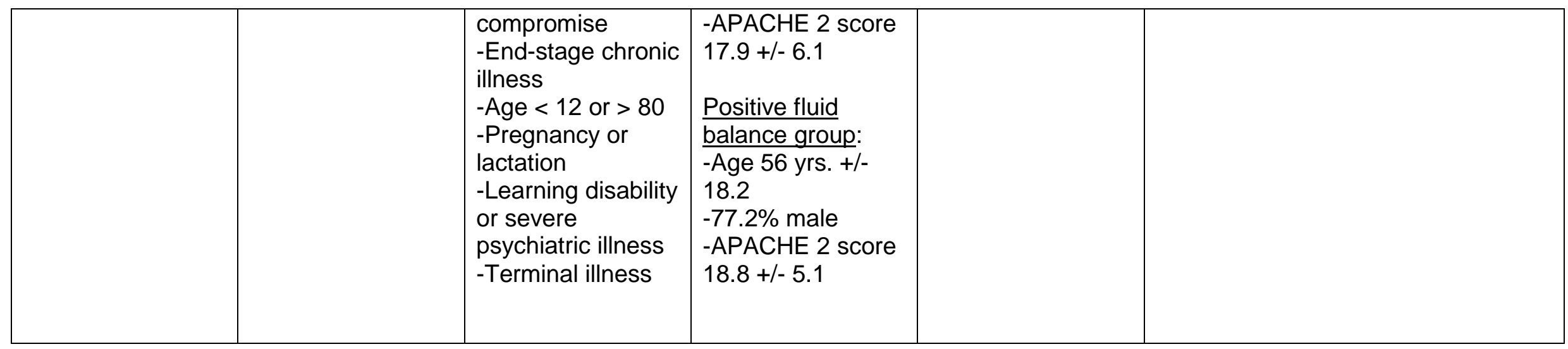


Appendix 2. Modified Newcastle-Ottawa scale for included cohort studies.

Selection:

1) Representativeness of the exposed cohort

a) truly representative of the average ARDS / SIRS / sepsis population in the community $\square$

b) somewhat representative of the average ARDS / SIRS / sepsis population in the community

c) selected group of patients

d) no description of the derivation of the cohort

2) Selection of the non exposed cohort
a) drawn from the same community as the exposed cohort
b) drawn from a different source

c) no description of the derivation of the non exposed cohort

3) Ascertainment of exposure
a) secure record (eg surgical records)
b) structured interview
c) written self report
d) no description

4) Demonstration that outcome of interest was not present at start of study
a) yes
b) no

Comparability:

1) Comparability of cohorts on the basis of the design or analysis

a) study controls for severity of illness

b) study controls for haemodynamic status

Outcome:

1) Assessment of outcome
a) independent blind assessment
b) record linkage
c) self report
d) no description

2) Was follow-up long enough for outcomes to occur
a) yes

b) no

3) Adequacy of follow up of cohorts

a) complete follow up - all subjects accounted for

b) subjects lost to follow up unlikely to introduce bias $>98 \%$ follow-up or description provided of those lost

c) follow up rate $<99 \%$ and no description of those lost

d) no statement 


\begin{tabular}{|c|c|c|c|}
\hline & Selection & Comparability & Outcome \\
\hline Abulebda et al, 2014 & ++++ & - & +++ \\
\hline Acheampong \& Vincent, 2015. & ++++ & + & +++ \\
\hline Bhaskar et al, 2015 & ++++ & ++ & +++ \\
\hline Bihari et al, 2013. & ++++ & ++ & ++ \\
\hline Botdorf et al, 2015. & +++ & - & + \\
\hline Boyd et al, 2011. & ++++ & ++ & ++ \\
\hline Chen et al, 2011. & +++ & + & + \\
\hline Cordemans et al, 2012. & ++ & ++ & + \\
\hline Cronhjort et al, 2016. & ++++ & ++ & +++ \\
\hline De Oliveira et al, 2015. & ++++ & + & ++ \\
\hline Fiorenza \& Pass, 2013. & +++ & - & + \\
\hline Flori et al, 2011. & ++++ & + & + \\
\hline Grissom et al, 2015. & +++ & + & +++ \\
\hline Herrera Gutierrez et al, 2013. & ++ & ++ & ++ \\
\hline Kongsayreepong \& Nitikaroon, 2013. & + & ++ & + \\
\hline Koonrangsesomboon \& Khwannimit, 2015 & ++++ & + & +++ \\
\hline Micek et al, 2013. & ++++ & + & ++ \\
\hline Murai et al, 2014 & +++ & - & + \\
\hline Murphy et al, 2009. & ++++ & ++ & ++ \\
\hline Perez-Fernandez et al, 2011. & +++ & - & + \\
\hline Raimundo et al, 2012. & +++ & - & + \\
\hline Rodriguez et al, 2013. & +++ & - & + \\
\hline Rosenberg et al, 2008. & ++++ & ++ & +++ \\
\hline Saito et al, 2012. & + & + & + \\
\hline Simmons et al, 1987. & +++ & - & ++ \\
\hline Smith \& Perner, 2012. & +++ & - & +++ \\
\hline Spicer et al, 2014. & + & + & + \\
\hline Sun et al, 2015. & +++ & + & +++ \\
\hline Udeozo et al, 2009. & +++ & - & + \\
\hline Valentine et al, 2012. & ++++ & ++ & + \\
\hline $\begin{array}{l}\text { Vincent et al, 2006. (sepsis) } \\
\text { Sakr et al , 2005. (ARDS) }\end{array}$ & $\begin{array}{l}++++ \\
++++\end{array}$ & $\begin{array}{l}++ \\
+\end{array}$ & $\begin{array}{l}++ \\
++\end{array}$ \\
\hline Wang et al, 2016. & +++ & + & ++ \\
\hline Wilkowski et al, 1988. & ++ & - & + \\
\hline Willson et al, 2015. & +++ & - & +++ \\
\hline Yao et al, 2014. & +++ & + & + \\
\hline Zhang et al, 2012. & +++ & ++ & ++ \\
\hline Zhang et al, 2013. & +++ & - & + \\
\hline
\end{tabular}


Appendix 3. Additional analyses.

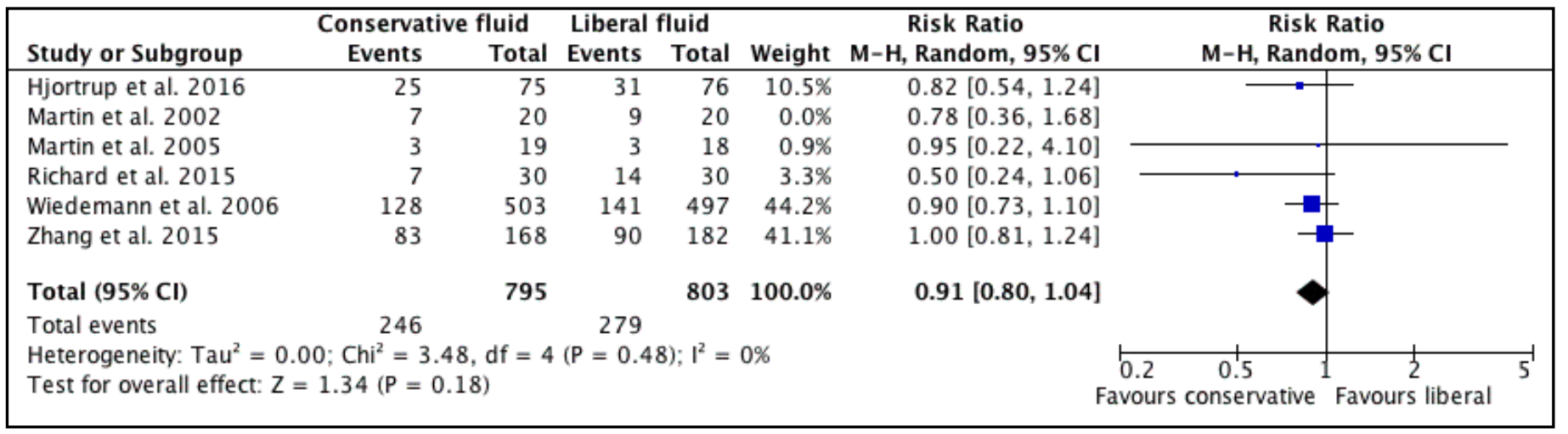

3.1 Pre-planned sensitivity analysis excluding studies at high or moderate risk of bias with mortality as outcome. 


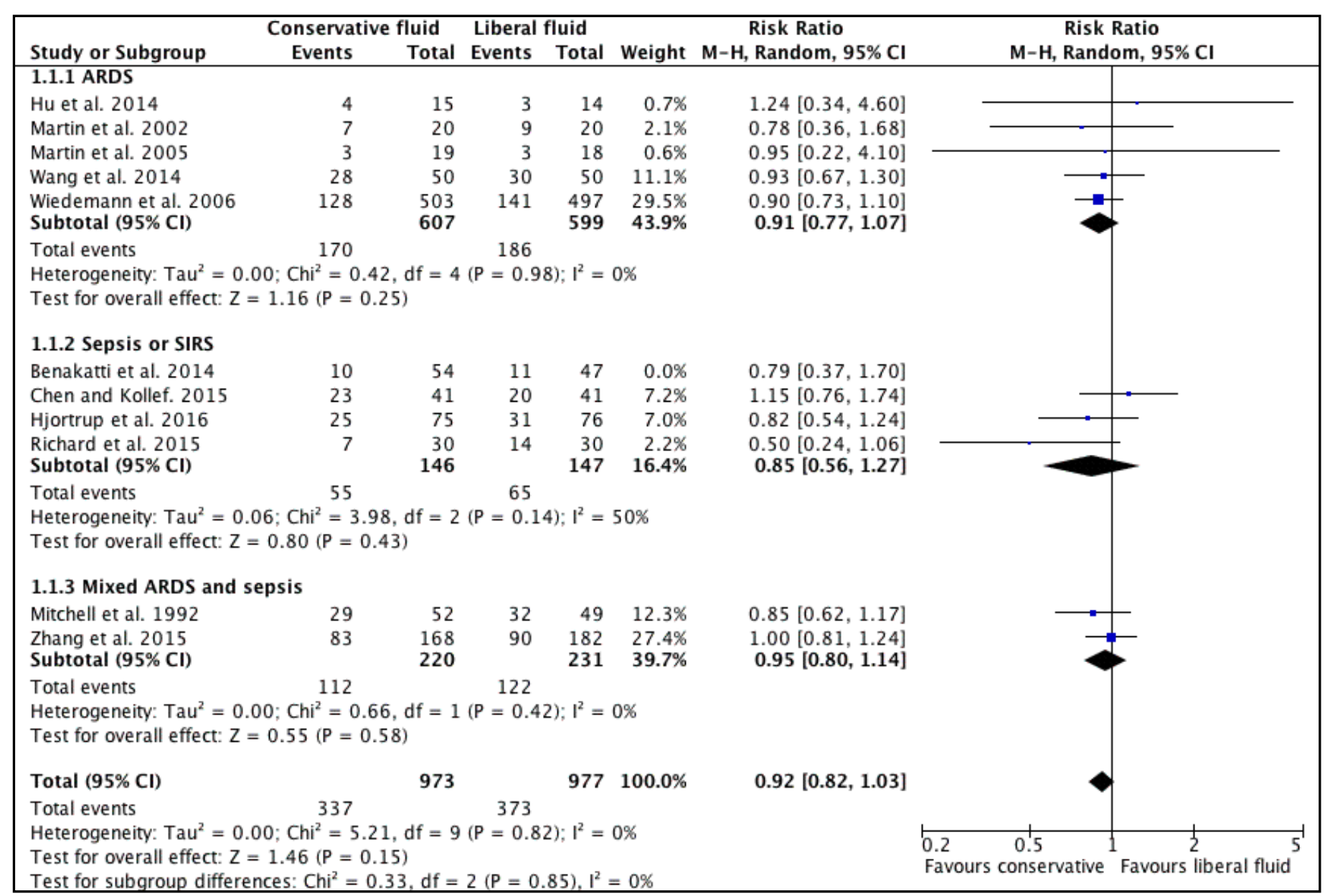

3.2 Pre-planned subgroup analysis including only adult studies with mortality as outcome. 


\begin{tabular}{|c|c|c|c|c|c|c|c|c|c|c|}
\hline \multirow{3}{*}{$\begin{array}{l}\text { Study or Subgroup } \\
\text { Benakatti et al. } 2014\end{array}$} & \multicolumn{2}{|c|}{ Conservative fluid } & \multicolumn{2}{|c|}{ Liberal fluid } & \multirow[b]{2}{*}{ Weight } & \multirow{2}{*}{$\begin{array}{c}\text { Risk Ratio } \\
\text { M-H, Random, } 95 \% \mathrm{Cl} \\
\end{array}$} & \multirow{2}{*}{\multicolumn{4}{|c|}{$\begin{array}{c}\text { Risk Ratio } \\
\text { M-H, Random, 95\% CI }\end{array}$}} \\
\hline & Events & Total & Events & Total & & & & & & \\
\hline & 10 & 54 & 11 & 47 & $3.6 \%$ & $0.79[0.37,1.70]$ & & & & \\
\hline Martin et al. 2002 & 7 & 20 & 9 & 20 & $3.5 \%$ & $0.78[0.36,1.68]$ & & & & \\
\hline Martin et al. 2005 & 3 & 19 & 3 & 18 & $1.0 \%$ & $0.95[0.22,4.10]$ & & & & \\
\hline Mitchell et al. 1992 & 29 & 52 & 32 & 49 & $20.6 \%$ & $0.85[0.62,1.17]$ & & 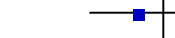 & - & \\
\hline Richard et al. 2015 & 7 & 30 & 14 & 30 & $3.6 \%$ & $0.50[0.24,1.06]$ & & & & \\
\hline Wang et al. 2014 & 28 & 50 & 30 & 50 & $18.5 \%$ & $0.93[0.67,1.30]$ & & & E & \\
\hline Wiedemann et al. 2006 & 128 & 503 & 141 & 497 & $49.3 \%$ & $0.90[0.73,1.10]$ & & & & \\
\hline Total $(95 \% \mathrm{Cl})$ & & 728 & & 711 & $100.0 \%$ & $0.87[0.75,1.00]$ & & & & \\
\hline Total events & 212 & & 240 & & & & & & & \\
\hline \multicolumn{6}{|c|}{$\begin{array}{l}\text { Heterogeneity: } \mathrm{Tau}^{2}=0.00 ; \mathrm{Chi}^{2}=2.50, \mathrm{df}=6(\mathrm{P}=0.87) ; \mathrm{I}^{2}=0 \% \\
\text { Test for overall effect: } \mathrm{Z}=1.93(\mathrm{P}=0.05)\end{array}$} & & $\begin{array}{l}0.2 \\
\text { Favours }\end{array}$ & $\begin{array}{c}0.51 \\
\text { conservative }\end{array}$ & $\frac{2}{1} \frac{2}{\text { Favours liberal }}$ & 5 \\
\hline
\end{tabular}

3.3 In a post-hoc sensitivity analysis in which we excluded studies lacking a clinically-important separation in fluid balance between groups, we found a nonsignificant reduction in mortality with a conservative or deresuscitative strategy compared to a liberal strategy or standard care.

We used these findings to calculate the required sample size to test the hypothesis that conservative fluid management or deresuscitation strategy reduces mortality compared to a liberal strategy or standard care. Based on a 13\% relative risk reduction (assuming a comparable difference in mortality to those studies in which a clinically-significant difference in fluid balance was achieved), a baseline mortality risk of 34\%, two-tailed alpha of 0.05 and power of $90 \%$, we calculated this to be 4704 patients. 


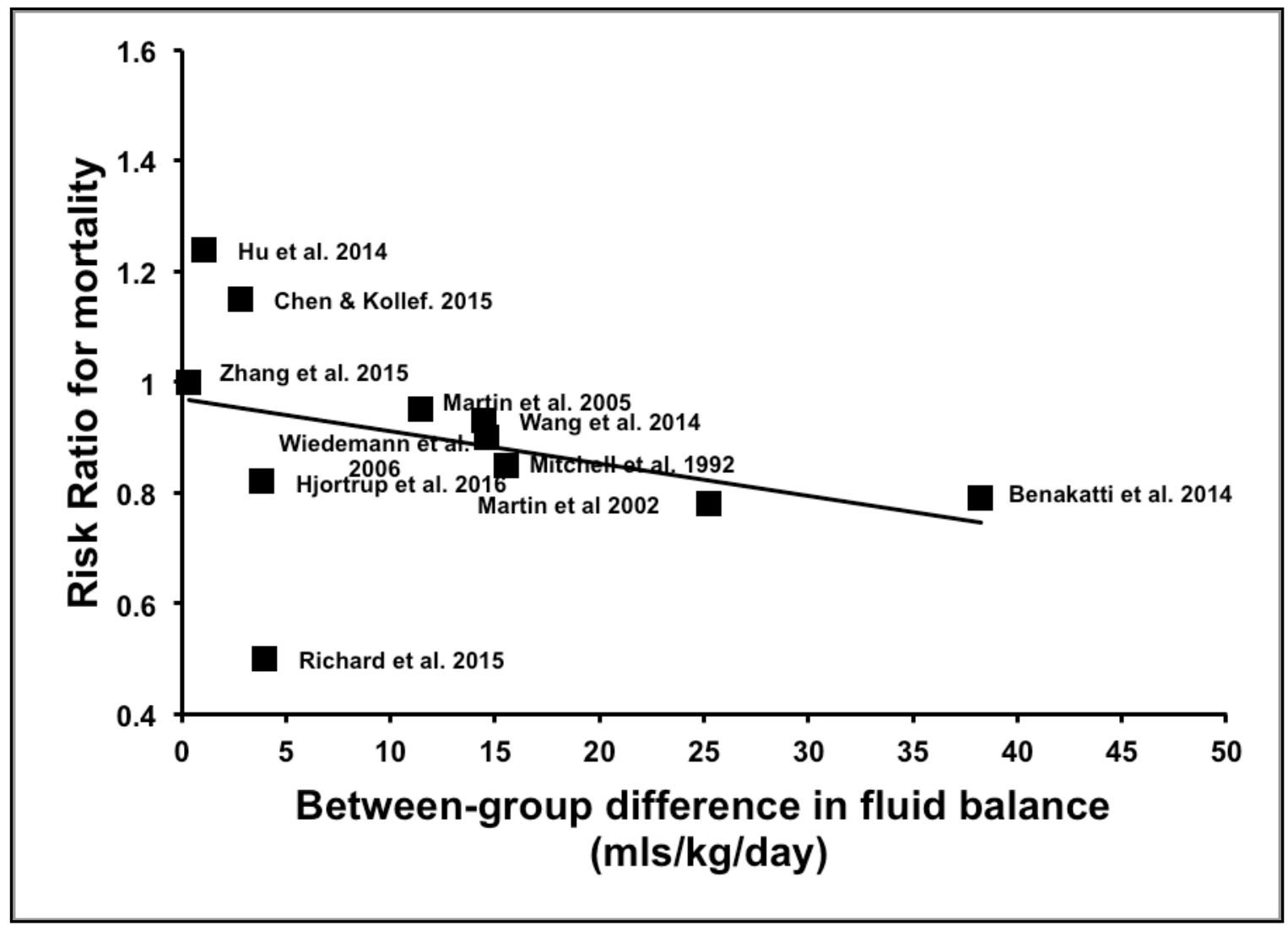

3.4 Univariate meta-regression analysis with RR for mortality as dependent variable and between-group difference in mean daily fluid balance as exposure. $\mathrm{R}^{2}=0.11, \mathrm{P}=0.30$ 


\begin{tabular}{|c|c|c|c|c|c|c|c|}
\hline \multirow[b]{2}{*}{ Study or Subgroup } & \multicolumn{2}{|c|}{ Conservative fluid } & \multicolumn{2}{|c|}{ Liberal fluid } & \multirow[b]{2}{*}{ Weight } & \multirow{2}{*}{$\begin{array}{c}\text { Risk Ratio } \\
\text { M-H, Random, 95\% CI } \\
\end{array}$} & \multirow{2}{*}{$\begin{array}{c}\text { Risk Ratio } \\
\text { M-H, Random, } 95 \% \mathrm{Cl} \\
\end{array}$} \\
\hline & Events & Total & Events & Total & & & \\
\hline Chen and Kollef. 2015 & 17 & 41 & 16 & 41 & $28.4 \%$ & $1.06[0.63,1.80]$ & $\longrightarrow$ \\
\hline Hjortrup et al. 2016 & 16 & 75 & 14 & 76 & $20.9 \%$ & $1.16[0.61,2.20]$ & 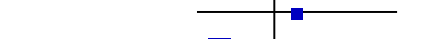 \\
\hline Wiedemann et al. 2006 & 50 & 503 & 70 & 497 & $50.7 \%$ & $0.71[0.50,0.99]$ & \\
\hline Total $(95 \% \mathrm{Cl})$ & & 619 & & 614 & $100.0 \%$ & $0.88[0.64,1.22]$ & \\
\hline \multirow{2}{*}{\multicolumn{6}{|c|}{$\begin{array}{l}\text { Total events } \\
\text { Heterogeneity: } \text { Tau }^{2}=0.02 ; \mathrm{Chi}^{2}=2.76, \mathrm{df}=2(\mathrm{P}=0.25) ; \mathrm{I}^{2}=27 \% \\
\text { Test for overall effect: } \mathrm{Z}=0.78(\mathrm{P}=0.44)\end{array}$}} & & \\
\hline & & & & & & & $\begin{array}{cccc}0.2 & 0.5 & 1 & 2 \\
\text { Favours conservative } & \text { Favours li }\end{array}$ \\
\hline
\end{tabular}

3.5 Forest plot for renal replacement therapy use, conservative or deresuscitative fluid strategy versus standard care or liberal fluid strategy. 
Aboelatta and Abdelsalam. Volume Overload of Fluid Resuscitation In Acutely Burned Patients Using Transpulmonary Thermodilution Technique. Journal of Burn Care and Research 2013;34:349-354

Acheampong and Vincent. Early Negative Fluid Balance Is Independently Associated With Improved Survival In Septic Patients. American Journal of Respiratory and Critical Care Medicine 2014;189;A5496

Acheampong and Vincent. Early Negative Fluid Balance Is Independently Associated With Improved Survival In Septic Patients. American Journal of Respiratory and Critical Care Medicine 2014;189;A5496

Acheampong and Vincent. A Positive Fluid Balance Is An Independent Prognostic Factor In Patients With Sepsis. Critical Care 2015;19:251

Aharoni et al. Burn Resuscitation With A Low-Volume Plasma Regimen--Analysis of Mortality. Burns 1989;15:230-232

Almeida et al. Impact of Positive Fluid Balance On Survival In Critically III Cancer Patients. Critical Care 2010;14(S1): S524

Alsous et al. Negative Fluid Balance Predicts Survival In Patients With Septic Shock: A Retrospective Pilot Study. Chest 2000;117:1749-1754

Andrews et al. Simplified Severe Sepsis Protocol: A Randomized Controlled Trial of Modified Early GoalDirected Therapy In Zambia. Critical Care Medicine 2014;42:2315-2324

Angelo et al. Fluid Status and Clinical Outcomes In Critically III Children With Sepsis: A Retrospective Analysis. Critical Care Medicine 2010;38(12 S1):386

Angelo et al. Fluid Status and Clinical Outcomes In Critically III Children With Sepsis: A Retrospective Analysis. Critical Care Medicine 2010;38(12 S1):386

ARISE Investigators. Goal-Directed Resuscitation For Patients With Early Septic Shock. The New England Journal of Medicine 2014;371:1496-1506

Arlati et al. Decreased Fluid Volume To Reduce Organ Damage: A New Approach To Burn Shock Resuscitation? A Preliminary Study. Resuscitation 2007;72:371-378

Azevedo et al. Should We Need Fluid Overload In the Critically III Patients? Nephrology Dialysis Transplantation 2013;28:I331

Azevedo et al. Association Between Early Fluid Balance, Organ Failures and Outcomes In Ventilated Patients. Critical Care Medicine 2015;43(12 S1):170
- Observational study with $\mathrm{n}<50$

- Duplicate / overlap

- Duplicate / overlap

- Duplicate / overlap

- Fluid type study

- Duplicate / overlap

- Observational study with $n<50$

- Complex haemodynamic intervention

- Resuscitation phase study

- Duplicate / overlap

- Duplicate / overlap

- Resuscitation phase study

- Complex haemodynamic intervention

- Observational study with $\mathrm{n}<50$

- Resuscitation phase study

- Study population did not match criteria

- Study population did not match criteria 
Balakumar et al. Fluid Balance Has Variable Association With Long-Term Survival In the Critically III. Critical Care Medicine 2015;43(12 S1):244

Bataller et al. Goal Directed Fluid Management In Resuscitated Sepsis: Evaluation of An Algorithm Based On Volumetric Parameters. European Journal of Anaesthesiology 2011;28:181

Bayer et al. Fluid Balance In Septic Patients Receiving Only Crystalloid Or Synthetic Fluids Plus Crystalloids. Intensive Care Medicine 2010;36:0926

Bayer et al. Effects of Fluid Resuscitation With Synthetic Colloids Or Crystalloids Alone On Shock Reversal, Fluid Balance, and Patient Outcomes In Patients With Severe Sepsis: A Prospective Sequential Analysis. Critical Care Medicine 2012; 40:2543-2551

Benakatti et al. Conventional Vs. Restrictive Maintenance Fluid Regime In Children With Septic Shock After Initial Resuscitation: A Randomized Open Label Controlled Trial. Archives of Disease In Childhood 2012;97:A5

Bhaskar et al. Early Fluid Accumulation In Children With Shock and ICU Mortality: A Matched CaseControl Study. Intensive Care Medicine 2015;41:8

Bhaskar et al. Impact of Early Fluid Overload On Mortality In Critically III Children - A Nested CaseControl Study. Critical Care Medicine 2013;41(12 S1):1000

Bihari et al. Post Resuscitation Fluid Boluses In Severe Sepsis Or Septic Shock: Prevalence and Efficacy (PRICE Study). American Journal of Respiratory and Critical Care Medicine 2013;187:A3952

Bocanegra et al. A Long-Term Study of Early Fluid Therapy In Severely Burned Adults. 3. Simultaneous Comparison of Saline Solution Alone Or Combined With Plasma. Jama : The Journal of The American Medical Association 1966;195:268-274

Botdorf et al. Retrospective Analysis of Fluid Balance In The First 48 Hours of Sepsis and Outcome. American Journal of Respiratory and Critical Care Medicine 2015;191:A4497

Boyd et al. Over-Resuscitation With Fluid In Septic Shock Decreases Survival and Increases Time On The Ventilator. American Journal of Respiratory and Critical Care Medicine 2010;181:A1137

Boyd et al. Over-Resuscitation With Fluid In Septic Shock Decreases Survival and Increases Time On The Ventilator. American Journal of Respiratory and Critical Care Medicine 2010;181:A1137
- Study population did not match criteria

- Observational study with $\mathrm{n}<50$

- Observational study with $\mathrm{n}<50$

- Fluid type study

- Fluid type study

- Duplicate / overlap

- Duplicate / overlap

- Duplicate / overlap

- Duplicate / overlap

- Pre-1980

- Resuscitation phase study

- Duplicate / overlap

- Duplicate / overlap

- Duplicate / overlap 
Carcillo et al. Role of Early Fluid Resuscitation In Pediatric Septic Shock. JAMA 1991;266:1242-1245 Carlsen and Perner. Initial Fluid Resuscitation of Patients With Septic Shock In The ICU. Acta Anaesthesiologica Scandinavica, Suppement 2009; 53 (119):72

Carlsen et al. Initial Fluid Resuscitation of Patients With Septic Shock In The Intensive Care Unit. Acta Anaesthesiologica Scandinavica 2011;55:394-400

Chang et al. Volume of Fluids Administered During Resuscitation For Severe Sepsis and Septic Shock and The Development of The Acute Respiratory Distress Syndrome. Journal of Critical Care 2014;29:1011-1015

Chen and Kollef. Fluid Balance In The ICU-Interventions To Minimize Fluids In Patients With Septic Shock. American Journal of Respiratory and Critical Care Medicine 2015;191:A5218

Chen and Kollef. Targeted Fluid Minimization Following Initial Resuscitation In Septic Shock: A Pilot Study. Chest 2015; 148(6):1462-9

Chen et al. The Impact of Different Fluid Management On Mortality In Patients With Septic Shock. Chinese Critical Care Medicine 2011;23:142-145

Cordemans et al. Effect of Negative Fluid Balance With PAL Therapy (Peep + Albumin + Lasix) On Capillary Leak Index, Intra-Abdominal and Abdominal Perfusion Pressure, Extravascular Lung Water and Organ Function In Acute Lung Injury. Critical Care 2010:36(S2);97

Cordemans et al. Effect of Negative Fluid Balance With PAL Therapy (Peep + Albumin + Lasix) On Capillary Leak Index, Intra-Abdominal and Abdominal Perfusion Pressure, Extravascular Lung Water and Organ Function In Acute Lung Injury. Intensive Care Medicine 2010;36(S2):97

Cordemans et al. Aiming For A Negative Fluid Balance In Patients With Acute Lung Injury and Increased Intra-Abdominal Pressure: A Pilot Study Looking At The Effects of PAL-Treatment. Annals of Intensive Care 2012;2 (S1):S15

Cordemans et al. Aiming For A Negative Fluid Balance In Patients With Acute Lung Injury and Increased Intraabdominal Pressure: A Pilot Study Looking At The Effects of PAL-Treatment. Annals of Intensive Care 2012;2(S1):S15

Crowe et al. Evaluation of A Modified Early Goal-Directed Therapy Protocol. American Journal of Emergency Medicine 2010;28:689-693

Cuartero et al. Negative Fluid Balance 48 Hours After Admission Improves Survival At 28 Days In Critically III Patients. Critical Care 2012;16(S1):P241

Cuartero et al. Negative Fluid Balance $48 \mathrm{H}$ After Admission Improves Survival At 28 Days In Critically III Patients. Critical Care 2012;16(S1):P241
- Resuscitation phase study

- Duplicate / overlap

- Resuscitation phase study

- Resuscitation phase study

- Duplicate / overlap

- Duplicate / overlap

- Duplicate / overlap

- Duplicate / overlap

- Fluid type study

- Duplicate / overlap

- Fluid type study

- Duplicate / overlap

- Fluid type study

- Duplicate / overlap

- Fluid type study

- Resuscitation phase study

- Study population did not match criteria

- Duplicate / overlap 
Cuartero et al. Negative Fluid Balance $48 \mathrm{H}$ After Admission Improves Survival At 28 Days In Critically III Patients. Intensive Care Medicine 2012;38(S1):0167

Cuartero et al. Negative Fluid Balance $48 \mathrm{H}$ After Admission Improves Survival At 28 Days In Critically III Patients. Intensive Care Medicine 2012;38(S1):0167

De Almeida et al. Positive Fluid Balance Is Associated With Reduced Survival In Critically III Patients With Cancer. Acta Anaesthesiologica Scandinavica 2012;56:712-717

De Oliveira et al. Positive Fluid Balance As A Prognostic Factor For Mortality and Acute Kidney Injury In Severe Sepsis and Septic Shock. Journal of Critical Care 2015;30(1):97-101

Depaye et al. Impact of Fluid Resuscitation Volume On The Severity of Organ Failures In Severely Burned Patients. Critical Care 2012;16(S1):P461

Diaz-Rubia et al. Effect of Maintained Negative Fluid Balance In Hypoxemic and High Extravascular Lung Water Patients. Intensive Care Medicine 2011;37(S1):0370

Diaz-Rubia et al. Effect of Maintained Negative Fluid Balance In Hypoxemic and High Extravascular Lung Water Patients. Intensive Care Medicine 2011;37(S1):0370

Dulhunty et al. Increased Fluid Resuscitation Can Lead To Adverse Outcomes In Major-Burn Injured Patients, But Low Mortality Is Achievable. Burns 2008;34:1090-1097

Early Goal-Directed Therapy Collaborative Group of Zhejiang. The Effect of Early Goal-Directed

Therapy On Treatment of Critical Patients With Severe Sepsis/Septic Shock: A Multi-Center,

Prospective, Randomized, Controlled Study. Chinese Critical Care Medicine 2010;22:331-334

Eisenberg et al. A Prospective Study of Lung Water Measurements During Patient Management In An Intensive Care Unit. Am Rev Respir Dis 1987;136:662-668

El-Akabawy et al. The Concept of Early Goal-Directed Therapy In Sepsis Syndrome. Intensive Care Medicine 2011;37(S1):0439

Finfer et al. A Comparison of Albumin and Saline For Fluid Resuscitation In The Intensive Care Unit. The New England Journal of Medicine 2004;350:2247-2256

Finfer et al. Impact of Albumin Compared To Saline On Organ Function and Mortality of Patients With Severe Sepsis. Intensive Care Medicine 2011;37:86-96

Flori et al. Positive Fluid Balance Is Associated With Higher Mortality and Prolonged Mechanical Ventilation In Pediatric Patients With Acute Lung Injury. Critical Care Research and Practice 2011:854142
- Duplicate / overlap

- Duplicate / overlap

- Study population did not match criteria

- Duplicate / overlap

- Resuscitation phase study

- Observational study with $\mathrm{n}<50$

- Duplicate / overlap

- Resuscitation phase study

- Resuscitation phase study

- Fluid balance not reported

- Study population did not match criteria

- Complex haemodynamic intervention

- Resuscitation phase study

- Fluid type study

- Fluid type study

- Duplicate / overlap

- Duplicate / overlap 
Garcia Olivares et al. Clinical Implication of Resuscitation With Fluids Guided By Central Venous Pressure In Septic Shock. Intensive Care Medicine 2010;36(S1):1349

Gardner et al. Faster Rate of Initial Fluid Resuscitation In Severe Acute Pancreatitis Diminishes InHospital Mortality. Pancreatology 2009;9:770-779.

Gomez et al. Late Fluid Management and Risk of Respiratory Failure In Septic Patients. American Journal of Respiratory and Critical Care Medicine 2009:179:A4730

Grams et al. Fluid Balance, Diuretic Use, and Mortality In Acute Kidney Injury. Clinical Journal of The American Society of Nephrology: CJASN 2011;6:966-973

Grams et al. Fluid Balance, Diuretic Use, and Mortality In Acute Kidney Injury. Clinical Journal of The American Society of Nephrology: CJASN 2011;6:966-973

Grissom et al. Retrospective Comparison of A Simpler Modified Fluid Conservative Protocol With The Original Factt Fluid Conservative Protocol In The ARDS Network. American Journal of Respiratory and Critical Care Medicine 2013;187:A4482

Grissom et al. Retrospective Comparison of A Simpler Modified Fluid Conservative Protocol With The Original Factt Fluid Conservative Protocol In The ARDS Network. American Journal of Respiratory and Critical Care Medicine 2013;187:A4482

Gu et al. Effect of Fluid Resuscitation On Extravascular Lung Water In Early Stage of Septic Shock. Chinese Critical Care Medicine 2007;19:283-286

Guidet et al. Assessment of Hemodynamic Efficacy and Safety of $6 \%$ Hydroxyethylstarch 130/0.4 Vs. $0.9 \% \mathrm{NaCl}$ Fluid Replacement In Patients With Severe Sepsis: The CRYSTMAS Study. Critical Care 2012;16:R94

Guidet et al. Assessment of Hemodynamic Efficacy and Safety of 6\% Hydroxyethylstarch 130/0.4 Vs. $0.9 \% \mathrm{NaCl}$ Fluid Replacement In Patients With Severe Sepsis: The CRYSTMAS Study. Critical Care 2012;16:R94

Heung et al. Fluid Overload At Initiation of Renal Replacement Therapy Is Associated With Lack of Renal Recovery In Patients With Acute Kidney Injury. Nephrology Dialysis Transplantation 2012:27:956961

Hjortrup et al. Patient and Site Characteristics and Volumes of Resuscitation Fluids In Severe Sepsis-A Post Hoc Analysis of A Randomised Clinical Trial. Intensive Care Medicine 2014;40(S1):0779

Hou et al. Positive Fluid Balance Worsens Clinical Outcomes For Hospitalized Pneumonia Patients. American Journal of Respiratory and Critical Care Medicine 2013;187:A5985
- Resuscitation phase study

- Observational study with $\mathrm{n}<50$

- Resuscitation phase study

- Duplicate / overlap

- Duplicate / overlap

- Duplicate / overlap

- Duplicate / overlap

- Resuscitation phase study

- Observational study with $\mathrm{n}<50$

- Fluid type study

- Fluid type study

- Duplicate / overlap

- Study population did not match criteria

- Clinical outcomes of interest not reported

- Study population did not match criteria

- Resuscitation phase study 
Hu et al. Extravascular Lung Water and Pulmonary Arterial Wedge Pressure For Fluid Management In Patients With Acute Respiratory Distress Syndrome. Multidisciplinary Respiratory Medicine 2014;9(1):3

Hunter et al. Fluid Creep In Colloid Resuscitated Burns Patients: Is Less More? Burns 2009;35:S14

Jacob et al. Severe Sepsis In Two Ugandan Hospitals: A Prospective Observational Study of Management and Outcomes In A Predominantly HIV-1 Infected Population. PLoS One 2009;4:E7782 Jelenko et al. Shock and Resuscitation. li: Volume Repletion With Minimal Edema Using The HALFD(Hypertonic Albuminated Fluid Demand) Regimen. Jacep 1978;7:326-333.

Jelenko et al. Studies In Shock and Resuscitation, I: Use of A Hypertonic, Albumin-Containing, Fluid Demand Regimen (HALFD) In Resuscitation. Critical Care Medicine 1979;7:157-167

Jelenko et al. Shock and Resuscitation Ii: Volume Repletion With Minimal Edema Using The 'Halfd' Method. Journal of The American College of Emergency Physicians 1978;7:326-333

Jog et al. Stroke Volume Variation-Guide To Fluid Therapy In Septic Shock With ARDS. Intensive Care Medicine 2009; 35(S1):0098

Jog et al. Stroke Volume Variation Guided Fluid Therapy In Septic Shock With ARDS. Critical Care 2012;16(S1):P233

Jog et al. Stroke Volume Variation Guided Fluid Therapy In Severe Septic Shock: When To Stop Chasing High SVV? Intensive Care Medicine 2013;39(S2):0084

Jones et al. Prospective External Validation of The Clinical Effectiveness of An Emergency DepartmentBased Early Goal-Directed Therapy Protocol For Severe Sepsis and Septic Shock. Chest 2007;132:425-432

Jones et al. Lactate Clearance Versus Central Venous Oxygen Saturation As Endpoints of Early Sepsis Therapy: A Randomized Clinical Trial. Critical Care Medicine 2009; 37(12 Supp.):49

Juneja et al. Use of Stroke Volume Variation To Guide Fluid Therapy In Septic Shock For Prevention of Acute Kidney Injury. Intensive Care Medicine 2009;35(S1):0104

Kalra et al. Fluid Balance At 72 Hours In Severe Sepsis and Septic Shock Patients Treated With Early Goal-Directed Therapy (EGDT) Is Associated With Increased Length of Mechanical Ventilation. Chest 2010;138 (4):A388

Kanji et al. Limited Echocardiography-Guided Therapy In Subacute Shock Is Associated With Change In Management and Improved Outcomes. Journal of Critical Care 2014;29:700-705
- Study population did not match criteria

- Clinical outcomes of interest not reported

- Resuscitation phase study

- Pre-1980

- Fluid type study

- Pre-1980

- Fluid type study

- Pre-1980

- Fluid type study

- Observational study with $n<50$

- Observational study with $\mathrm{n}<50$

- Observational study with $\mathrm{n}<50$

- Resuscitation phase study

- Complex haemodynamic intervention

- Resuscitation phase study

- Fluid balance not reported

- Fluid balance not reported

- Complex haemodynamic intervention 
Katsaragakis et al. Rapid Fluid Removal Via Continuous Venovenous Hemodiafiltration and Oxygen Delivery, Oxygen Consumption, and Outcome In Septic Patients With Renal Dysfunction. Dialysis and Transplantation 2005;34:608-615

Kaufman et al. Fluid Resuscitation In Circulatory Shock. Colloids Versus Crystalloids. Current Studies In Hematology \& Blood Transfusion 1986:186-198

Kelm et al. The Potential Burdens of Fluid Overload In Patients With Septic Shock. American Journal of Respiratory and Critical Care Medicine 2013;187:A3063

Kelm et al. Fluid Overload In Patients With Severe Sepsis and Septic Shock Treated With Early GoalDirected Therapy Is Associated With Increased Aute Need For Fluid-Related Medical Interventions and Hospital Death. Shock

2015;43(1):68-73

Kelm et al. Fluid Overload In Patients With Severe Sepsis and Septic Shock Treated With Early GoalDirected Therapy Is Associated With Increased Acute Need For Fluid-Related Medical Interventions and Hospital Death. Shock 2015;43:68-73

Kiers et al. Early Goal-Directed Therapy of Septic Patients Coming From The Ward Does Not Protect The Kidneys. Critical Care 2009;13(S1):P259

Kiers et al. Effect of Early Achievement of Physiologic Resuscitation Goals In Septic Patients Admitted From The Ward On The Kidneys. Journal of Critical Care 2010;25:563-569

Kim et al. A Positive Fluid Balance and Used Loop Diuretics Amount Are Associated Increased Mortality In Intensive Care Unit. Intensive Care Medicine 2012;38(S1):0911

Klein et al. The Association Between Fluid Administration and Outcome Following Major Burn: A Multicenter Study. Annals of Surgery 2007;245:622-628

Koonrangsesomboon and Khwannimit. Positive Fluid Balance and Prognostic Factors of ICU Mortality In Patients Admitted With Septic Shock. Critical Care 2014;18(S2):P70

Kraft et al. Outcomes of Optimized Fluid Resuscitation After Severe Burn Injury Utilizing Hemodynamic Measurements By Transpulmonary Thermodilution. Surgical Infections 2011;12:S16-S17

Krausz et al. Cardiopulmonary Effects of Volume Loading In Patients In Septic Shock. Annals of Surgery 1977; 185:429-434
- Observational study with $\mathrm{n}<50$

- Not original study

- Duplicate / overlap

- Duplicate / overlap

- Fluid balance not reported

- Resuscitation phase study

- Duplicate / overlap

- Resuscitation phase study

- Study population did not match criteria

- Resuscitation phase study

- Study population did not match criteria

- Fluid balance not reported

- Resuscitation phase study

- Observational study with $\mathrm{n}<50$

- Pre-1980

- Clinical outcomes of interest not reported 
Lau et al. Fluid Management For Shock In The First 24 Hours of Intensive Care Unit Stay: Impact of A Simple Clinical Protocol. Annals of The Academy of Medicine Singapore 2014;1:S319

Lechliter and Shibli. Impact of Fluid Management Strategies In Patients With Acute Respiratory Distress Syndrome (ARDS). Chest 2009;136(S4):S77

Lee et al. Association Between Fluid Balance and Survival In Critically III Patients. Journal of Internal Medicine 2015;277(4):468-77

Lee et al. Association Between Fluid Balance and Survival In Critically III Patients. Journal of Internal Medicine 2015;277(4):468-77

Lee et al. Is Fluid Administration Within Six Hours Early Enough For Better Patient Outcomes In Sepsis and Septic Shock? Critical Care Medicine 2012;40(12):26

Lee et al. Increased Fluid Administration In The First Three Hours of Sepsis Resuscitation Is Associated With Reduced Mortality: A Retrospective Cohort Study. Chest 2014;146:908-915

Legrand et al. A Role of Renal Venous Congestion In Septic Acute Kidney Injury? Intensive Care Medicine 2011;37(S1):0968

Lin et al. A Modified Goal-Directed Protocol Improves Clinical Outcomes In Intensive Care Unit Patients With Septic Shock: A Randomized Controlled Trial. Shock 2006;26:551-557

Liu et al. Acute Kidney Injury In Patients With Acute Lung Injury: Impact of Fluid Accumulation On Classification of Acute Kidney Injury and Associated Outcomes. Critical Care Medicine 2011; 39:26652671

Lobo and Cunha. Time To Recover From Shock Is Determinant of A Positive Fluid Balance In Septic Shock. Critical Care 2013:17(S2):P238

Lobo and Cunha. Time To Recover From Shock Is Determinant of A Positive Fluid Balance In Septic Shock. Critical Care 2013; 17(S2):P238

Lyu et al. Efficacies of Fluid Resuscitation As Guided By Lactate Clearance Rate and Central Venous Oxygen Saturation In Patients With Septic Shock. National Medical Journal of China 2015;95: 496-500

Mahrous et al. Renal Effect of Colloid Versus Crystalloid In Septic Neutropenic Patients. Critical Care Medicine 2013;41(12 S1):996

Maitland et al. Mortality After Fluid Bolus In African Children With Severe Infection. The New England Journal of Medicine 2011;364:2483-2495
- Resuscitation phase study

- Observational study with $\mathrm{n}<50$

- Study population did not match criteria

- Study population did not match criteria

- Resuscitation phase study

- Resuscitation phase study

- Fluid balance not reported

- Resuscitation phase study

- Complex haemodynamic intervention

- Duplicate / overlap

- Observational study with $\mathrm{n}<50$ patients.

- Duplicate / overlap

- Resuscitation phase study

- Fluid type study

- Resuscitation phase study 
Maitland et al. Exploring Mechanisms of Excess Mortality With Early Fluid Resuscitation: Insights From The FEAST Trial. BMC Medicine 2013;11:Pp

Mao et al. Therapeutic Experience of Fulminant Acute Pancreatitis In Acute Response Stage. Chinese Journal of Surgery 2006;44:1185-1188

Mao et al. Fluid Therapy For Severe Acute Pancreatitis In Acute Response Stage. Chinese Medical Journal 2009;122:169-173

Martin. Fluid Balance and Colloid Osmotic Pressure In Acute Respiratory Failure: Emerging Clinical Evidence. Critical Care 2000;4:S21-S25

Mcintyre et al. Resuscitating Patients With Early Severe Sepsis: A Canadian Multicentre Observational Study. Canadian Journal of Anaesthesia 2007;54:790-798

Mitchell et al. Volume Overload: Prevalence, Risk Factors, and Functional Outcome In Survivors of Septic Shock. Annals of The American Thoracic Society 2015;12(12):1837-44

Mitchell et al. Fluid Management of Sepsis After Shock Resolution: An Analysis of Intensive Care Unit Volume Status In Relation To Mobility On Hospital Discharge. American Journal of Respiratory and Critical Care Medicine 2013;187:A3951

Mitchell et al. Fluid Management of Sepsis After Shock Resolution: An Analysis of Practice Variation and Risk Factors. American Journal of Respiratory and Critical Care Medicine 2012;185:A1124

Morisawa et al. Combined Use of Transpulmonary Thermodilution Technique In Fluid Management For Sepsis Patients. Intensive Care Medicine 2014;40(S1):0147

Mouncey et al. Trial of Early, Goal-Directed Resuscitation For Septic Shock. New England Journal of Medicine 2015;372:1301-1311

Muller et al. Fluid Management and Risk Factors For Renal Dysfunction In Patients With Severe Sepsis and/Or Septic Shock. Critical Care 2012;16:R34

Ngo et al. Acute Management of Dengue Shock Syndrome: A Randomized Double-Blind Comparison of 4 Intravenous Fluid Regimens In The First Hour. Clinical Infectious Diseases 2001; 32:204-213

Nogueira et al. Positive Daily Fluid Balance As An Independent Predictor of Mortality In Critically III Patients. Intensive Care Medicine 2013;39(S2):0250

Oliveira et al. Early and Late Positive Fluid Balance In Sepsis: Are They Both Related To Mortality and Acute Kidney Injury? Critical Care Medicine 2011;12(S1):56

Oliveira et al. Early and Late Positive Fluid Balance In Sepsis: Are They Both Related To Mortality and Acute Kidney Injury? Critical Care Medicine 2011;12(S1):56
- Resuscitation phase study

- Not relevant

- Resuscitation phase study

- Not original study

- Resuscitation phase study

- Clinical outcomes of interest not reported

- Duplicate / overlap

- Duplicate / overlap

- Observational study with $\mathrm{n}<50$

- Complex haemodynamic intervention

- Resuscitation phase study

- Fluid type study

- Resuscitation phase study

- Study population did not match criteria

- Duplicate / overlap

- Duplicate / overlap 
Olupot-Olupot. Fluid Expansion As Supportive Therapy (Feast) Trial: Mortality After Fluid Bolus In African Children. Tropical Medicine and International Health 2012;17:43

Orbegozo et al. Colloids For Fluid Resuscitation: What Is Their Role In Patients With Shock? Minerva Anestesiologica 2014;80:963-969

Ozuzun et al. Early Fluid Resuscitation of End Stage Renal Disease Patients With Severe Sepsis and Septic Shock. Critical Care Medicine 2014;42(12):923

Park et al. Early Cumulative Fluid Balance and Development of Acute Lung Injury. American Journal of Respiratory and Critical Care Medicine 2011;183:A5592

Patolia et al. Predicting Clinical Deterioration In Severe Sepsis Patients With Cryptic Shock. Intensive Care Medicine 2014;40(S1):1025

Payen et al. A Positive Fluid Balance Is Associated With A Worse Outcome In Patients With Acute Renal Failure. Critical Care 2008;12:R74

Pereira et al. Impact of Fluid Balance To Organ Dysfunction In Critically III Patients. Critical Care 2016;20:P171

Perez-Fernandez et al. Fluid Balance On Early Stages of Septic Shock Patients With Continuous Renal Replacement Techniques. Intensive Care Medicine 2011;37(S1):0970

Permpikul et al. Impact of Septic Shock Hemodynamic Resuscitation Guidelines On Rapid Early Volume Replacement and Reduced Mortality. Journal of The Medical Association of Thailand 2010; 93 (S1):S102-S109

Perner et al. Long-Term Outcomes In Patients With Severe Sepsis Randomised To Resuscitation With Hydroxyethyl Starch 130/0.42 Or Ringer's Acetate. Intensive Care Medicine 2014;40:927-934

Phillips et al. Crystalloids Vs. Colloids: Ko At The Twelfth Round? Critical Care 2013;17:319

Puffinbarger et al. Rapid Isotonic Fluid Resuscitation In Pediatric Thermal Injury. Journal of Pediatric Surgery 1994;29:339-342

Puskarich et al. One Year Mortality of Patients Treated With An Emergency Department Based Early Goal Directed Therapy Protocol For Severe Sepsis and Septic Shock: A Before and After Study. Critical Care 2009;13:R167

Raimundo et al. Impact of Fluid Balance In The Outcome of Septic Critically III Patients. Intensive Care Medicine 2012;38(S1):0696

Recinos et al. Fluid Resuscitation of Burn Patients Comparing A Crystalloid With A Colloid Containing Solution: A Prospective Study. Journal of The lowa Medical Society 1975;65:426-432
- Not original study

- Fluid type study

- Resuscitation phase study

- Study population did not match criteria

- Resuscitation phase study

- Study population did not match criteria

- Study population did not match criteria

- Duplicate / overlap

- Resuscitation phase study

- Fluid type study

- Fluid type study

- Resuscitation phase study

- Resuscitation phase study

- Complex haemodynamic intervention

- Duplicate / overlap

- Pre-1980 
Reddy et al. Furosemide Infusion In Children With Dengue Fever and Hypoxemia. Indian Pediatrics 2014;51:303-305

Richard et al. Preload-Dependence Indices To Titrate Volume Expansion During Septic Shock: A Randomized Controlled Trial. Intensive Care Medicine 2014;40(S1):0881

Richard et al. Preload-Dependence Indices To Titrate Volume Expansion During Septic Shock: A Randomized Controlled Trial. Intensive Care Medicine 2014;40(S1):0881

Rinaldi et al. Effectiveness of Sepsis Bundle Application In Cirrhotic Patients With Septic Shock: A Single-Center Experience. Journal of Critical Care 2013;28:152-157

Rinka et al. Fluid Therapy In Patients With Severe Sepsis After The Earliest Phases of Treatment. Critical Care Medicine 2009: 37(12):913

Rinka et al. Fluid Therapy In Patients With Severe Sepsis After The Earliest Phases of Treatment. Critical Care Medicine 2009;37(12 S1): 913

Rivers et al. Early Goal-Directed Therapy In The Treatment of Severe Sepsis and Septic Shock. The New England Journal of Medicine 2001;345:1368-1377

Sabater et al. Diuretics In Septic Acute Renal Failure Needing Continuous Renal Replacement Therapies. Intensive Care Medicine 2011;37(S1):0977

Sadaka et al. Fluid Resuscitation In Septic Shock: The Effect of Increasing Fluid Balance On Mortality. Critical Care Medicine 2012;40(12 S1):533

Sadaka et al. Fluid Resuscitation In Septic Shock: The Effect of Increasing Fluid Balance On Mortality. Critical Care Medicine 2012;40(12 S1):533

Sadaka et al. Fluid Resuscitation In Septic Shock: The Effect of Increasing Fluid Balance On Mortality. Journal of Intensive Care Medicine 2014;29:213-217.

Saito et al. Implementation of An Optimal Fluid Management Protocol Using The PICCO System Delays Development of ARDS Secondary To Severe Sepsis. Critical Care 2012;16(S1):P243

Sakr et al. High Tidal Volume and Positive Fluid Balance Are Associated With Worse Outcome In Acute Lung Injury. Chest 2005;128:3098-3108.

Sammani et al. Positive Fluid Balance Is A Risk Factor For Acute Kidney Injury In Critically III Patients. Critical Care Medicine 2014;42(12 S1):928
- Fluid balance not reported

- Duplicate / overlap

- Duplicate / overlap

- Resuscitation phase study

- Observational study with $n<50$

- Fluid balance not reported

- Duplicate / overlap

- Resuscitation phase study

- Complex haemodynamic intervention

- Fluid balance not reported

- Resuscitation phase study

- Resuscitation phase study

- Duplicate / overlap

- Resuscitation phase study

- Duplicate / overlap

- Duplicate / overlap

- Study population did not match criteria 
Samransamruajkit et al. A Comparison of NSS vs Balanced Salt Solution As A Fluid Resuscitation and Impact of Fluid Balance On Clinical Outcomes In Pediatric Septic Shock. American Journal of

Respiratory and Critical Care Medicine 2015;191:A4072

Santhanam et al. A Prospective Randomized Controlled Study of Two Fluid Regimens In The Initial Management of Septic Shock In The Emergency Department. Pediatric Emergency Care 2008;24:647655

Schwab et al. Standard Operating Procedure In Patients With Severe Sepsis and Septic Shock. Critical Care 2009;13(S1):P343

Seymour et al. Prehospital Intravenous Access and Fluid Resuscitation In Severe Sepsis: An Observational Cohort Study. Critical Care 2014;18:533

Shimazaki et al. Fluid Distribution and Pulmonary Dysfunction Following Burn Shock. Journal of Trauma-Injury Infection \& Critical Care 1991;31:623-626

Silversides et al. Fluid Balance and Renal Outcomes In Patients Requiring Renal Replacement Therapy In The ICU. Critical Care 2013;17(S2):P434

Sirvent et al. Fluid Balance In Sepsis and Septic Shock As A Determining Factor of Mortality. American Journal of Emergency Medicine 2015;33:186-189

Sirvent et al. Fluid Balance In Sepsis and Septic Shock As A Determining Factor of Mortality. The American Journal of Emergency Medicine. 2015;33(2):186-9

Sivayoham et al. Outcomes From Implementing Early Goal-Directed Therapy For Severe Sepsis and Septic Shock : A 4-Year Observational Cohort Study. European Journal of Emergency Medicine 2012;19:235-240

Smith and Perner. Lower Mortality With Higher Fluid Volume In Patients With Persisting Septic Shock. Critical Care 2011:37(S1):905

Smith et al. Higher Vs. Lower Fluid Volume For Septic Shock: Clinical Characteristics and Outcome In Unselected Patients In A Prospective, Multicenter Cohort. Critical Care 2012;16:R76

Somasetia et al. Early Resuscitation of Dengue Shock Syndrome In Children With Hyperosmolar Sodium-Lactate: A Randomized Single-Blind Clinical Trial of Efficacy and Safety. Critical Care 2014;18:466

Sterling et al. The Effect of Early Quantitative Resuscitation On Organ Function In Survivors of Septic Shock. Journal of Critical Care 2015;30:261-263
- Observational study with $\mathrm{n}<50$

- Fluid type study

- Resuscitation phase study

- Resuscitation phase study

- Duplicate / overlap

- Study population did not match criteria

- Observational study with $n<50$

- Observational study with $\mathrm{n}<50$

- Duplicate / overlap

- Resuscitation phase study

- Duplicate / overlap

- Duplicate / overlap

- Resuscitation phase study

- Fluid type study

- Resuscitation phase study 
Stewart et al. Less Is More: Improved Outcomes In Surgical Patients With Conservative Fluid Administration and Central Venous Catheter Monitoring. Journal of The American College of Surgeons 2009; 208:725-735

Stewart et al. Less Is More: Improved Outcomes In Surgical Patients With Conservative Fluid Administration and Central Venous Catheter Monitoring. Journal of The American College of Surgeons 2009;208:725-735

Sun et al. The Clinical Application of Pulse Indicator Continuous Cardiac Output Monitoring In Early Fluid Resuscitation For Patients With Severe Acute Pancreatitis. Zhonghua Wei Zhong Bing Ji Jiu Yi Xue 2014;26:571-575

Toy et al. Transfusion-Related Acute Lung Injury: Incidence and Risk Factors. Blood 2012;119:17571767

Trof et al. Volume-Versus Pressure-Guided Hemodynamic Management In The Critically III Septic and Non-Septic Patient: Transpulmonary Thermodilution With PICCO Technology Versus Pulmonary Artery Catheter. Intensive Care Medicine 2010; 36(S1):1052

Trof et al. Volume-Limited Versus Pressure-Limited Hemodynamic Management In Septic and Nonseptic Shock. Critical Care Medicine 2012;40:1177-1185

Uchino et al. Pulmonary Artery Catheter Versus Pulse Contour Analysis: A Prospective Epidemiological Study. Critical Care 2006;10:R174

Vaara et al. Fluid Overload Is Associated With An Increased Risk For 90-Day Mortality In Critically III Patients With Renal Replacement Therapy: Data From The Prospective Finnaki Study. Critical Care 2012;16:R197

Valentine et al. Fluid Balance In Critically III Children With Acute Lung Injury Approximates The ARDSnet Fluid and Catheter Treatment Trial (Factt) Liberal Fluid Protocol and Is Associated With A Longer Duration of Mechanical Ventilation. American Journal of Respiratory and Critical Care Medicine 2011;183:A6280

Van Biesen et al. Relationship Between Fluid Status and Its Management On Acute Renal Failure (Arf) In Intensive Care Unit (ICU) Patients With Sepsis: A Prospective Analysis. Journal of Nephrology 2005;18:54-60

Van Genderen et al. Early Peripheral Perfusion Targeted Fluid Therapy Leads To Less Fluid Administration In Patients With Septic Shock: A Prospective Randomized Controlled Trial. Intensive Care Medicine 2014:40(S1):0452

Van Der Voort et al. Testing A Conceptual Model On Early Opening of The Microcirculation In Severe Sepsis and Septic Shock: A Randomised Controlled Pilot Study. European Journal of Anaesthesiology 2015;32:189-198
- Duplicate / overlap

- Duplicate / overlap

- Observational study with $\mathrm{n}<50$

- Not relevant

- Resuscitation phase study

- Resuscitation phase study

- Study population did not match criteria

- Study population did not match criteria

- Duplicate / overlap

- Fluid balance not reported

- Resuscitation phase study

- Complex haemodynamic intervention 
Vladimir and Niksa . Delivery Dependent Oxygen Consumption In Patients With ARDS: Relationship Between Volume Loading and Outcome. Clinical Intensive Care 1992;3:42

Waechter et al. Early Administration of Crystalloid Fluids Reduces Mortality In Septic Shock. American Journal of Respiratory and Critical Care Medicine 2010;181:A4097

Waechter et al. Interaction Between Fluids and Vasoactive Agents On Mortality In Septic Shock: A Multicenter, Observational Study. Critical Care Medicine 2014;42:2158-2168

Warndorf et al. The Effect of Early Aggressive Fluid Resuscitation On Morbidity and Mortality In Acute Pancreatitis. Gastroenterology 2011;140(5):S13

Wiedemann et al. Comparison of Two Fluid-Management Strategies In Acute Lung Injury. The New England Journal of Medicine 2006;354:2564-2575

Williams et al. Implementation of Early Goal-Directed Therapy For Children With Suspected Sepsis: Minimizing Unnecessary Variation and Maximizing Benefit. Critical Care Medicine 2010;38(12 S1):62

Yan. The Effect of Early Goal-Directed Therapy On Treatment of Critical Patients With Severe Sepsis/Septic Shock: A Multi-Center, Prospective, Randomized, Controlled Study. Chinese Critical Care Medicine 2010;22:331-334

Yang et al. Effects of Early Goal-Directed Fluid Therapy on Intra-abdominal Hypertension and Multiple Organ Dysfunction in Patients with Severe Acute Pancreatitis. Chinese Journal of Surgery 2009; 47:1450-1454

Yu et al. Comparison of The Effect of Fluid Resuscitation As Guided Either By Lactate Clearance Rate Or By Central Venous Oxygen Saturation In Patients With Sepsis. Chinese Critical Care Medicine 2013;25(10):578-583

Yu et al. Comparison of The Effect of Fluid Resuscitation As Guided Either By Lactate Clearance Rate Or By Central Venous Oxygen Saturation In Patients With Sepsis. Chinese Critical Care Medicine 2013;25(10):578-583

Zhang et al. Comparative Study On The Effect of Restrictive Fluid Management Strategy On The Early Pulmonary Function of Patients With Severe Burn. Chinese Journal of Burns 2012;28:165-169

Zhang et al. The Influence of Volume Load On Prognosis of Patients With Sepsis Induced Acute Kidney Injury. Zhonghua Wei Zhong Bing Ji Jiu Yi Xue 2013;25:411-414

Zhang et al. Effectiveness of Treatment Based On PICCO Parameters In Critically III Patients With Septic Shock and/Or Acute Respiratory Distress Syndrome: A Randomized Controlled Trial. Intensive Care Medicine 2015;41:444-451
- Observational study with $n<50$

- Resuscitation phase study

- Resuscitation phase study

- Study population did not match criteria

- Duplicate / overlap

- Resuscitation phase study

- Complex haemodynamic intervention

- Complex haemodynamic intervention

- Resuscitation phase study

- Fluid type study

- Study population did not match criteria

- Study population did not match criteria

- Observational study with $n<50$

- Duplicate / overlap

- Duplicate / overlap 
Zhao et al. Effects of Different Resuscitation Fluid On Severe Acute Pancreatitis. World Journal of Gastroenterology 2013;19:2044-2052

Zhi et al. Influence of Fluid Loading In Patients At Different Stages of Septic Shock: A Prospective Study. Zhonghua Wei Zhong Bing Ji Jiu Yi Xue 2015;27:13-16

Zhu et al. The Study of Hypertonic Saline and Hydroxyethyl Starch Treating Severe Sepsis. Chinese Critical Care Medicine 2011;23:150-153
- Fluid type study

- Observational study with $\mathrm{n}<50$

- Fluid type study 